\title{
Typing Messages for Free in Security Protocols
}

\author{
RÉMY CHRÉTIEN, Independent researcher, France \\ VÉRONIQUE CORTIER, LORIA, CNRS, France \\ ANTOINE DALLON, LSV, CNRS \& ENS Paris-Saclay, Université Paris-Saclay, France \\ STÉPHANIE DELAUNE, Univ Rennes, CNRS, IRISA
}

\begin{abstract}
Security properties of cryptographic protocols are typically expressed as reachability or equivalence properties. Secrecy and authentication are examples of reachability properties, while privacy properties such as untraceability, vote secrecy, or anonymity are generally expressed as behavioral equivalence in a process algebra that models security protocols.

Our main contribution is to reduce the search space for attacks for reachability as well as equivalence properties. Specifically, we show that if there is an attack then there is one that is well-typed. Our result holds for a large class of typing systems, a family of equational theories that encompasses all standard primitives, and protocols without else branches. For many standard protocols, we deduce that it is sufficient to look for attacks that follow the format of the messages expected in an honest execution, therefore considerably reducing the search space.
\end{abstract}

CCS Concepts: • Security and privacy $\rightarrow$ Logic and verification;

Additional Key Words and Phrases: Security protocols, symbolic model, verification, trace equivalence

ACM Reference format:

Rémy Chrétien, Véronique Cortier, Antoine Dallon, and Stéphanie Delaune. 2019. Typing Messages for Free in Security Protocols. ACM Trans. Comput. Logic 21, 1, Article 1 (September 2019), 52 pages.

https://doi.org/10.1145/3343507

\section{INTRODUCTION}

Formal methods have been very successful for the analysis of security protocols and many decision procedures and tools (e.g., References $[27,39,40]$ ) have been proposed. Two main families of security properties are typically considered: trace or accessibility properties, as well as equivalence properties. The former are used to express the most standard properties such as secrecy and authentication: for any execution of the protocol, an attacker should not learn the secret nor get authenticated without the server having accepted her request. The latter model privacy properties such as untraceability, vote secrecy, or anonymity (e.g., References [9, 17]). For example,

This work has been partially supported by the European Research Council (ERC) under the European Union's Horizon 2020 research and innovation program (grant agreements No. 645865-SPOOC and No. 714955-POPSTAR, the ANR project TECAP No. ANR-17-CE39-0004-01, and the DGA.

Authors' addresses: R. Chrétien and A. Dallon, LSV, CNRS \& ENS Paris-Saclay, 61, avenue du Président Wilson, 94235 CACHAN Cedex, France; email: dallon@lsv.fr; V. Cortier, LORIA, Campus Scientifique, F54506 Vandœuvre lès Nancy; email: veronique.cortier@loria.fr; S. Delaune, Campus universitaire de Beaulieu, 263 Avenue du Général Leclerc, 35042 Rennes Cedex - France; email: stephanie.delaune@irisa.fr.

Permission to make digital or hard copies of all or part of this work for personal or classroom use is granted without fee provided that copies are not made or distributed for profit or commercial advantage and that copies bear this notice and the full citation on the first page. Copyrights for components of this work owned by others than ACM must be honored. Abstracting with credit is permitted. To copy otherwise, or republish, to post on servers or to redistribute to lists, requires prior specific permission and/or a fee. Request permissions from permissions@acm.org.

(C) 2019 Association for Computing Machinery.

1529-3785/2019/09-ART1 $\$ 15.00$

https://doi.org/10.1145/3343507 
the anonymity of Bob is typically expressed by the fact that an adversary should not distinguish between the situation where Bob is present and the situation where Alice is present. Formally, the behavior of a protocol can be modeled through a process algebra such as CSP or the pi calculus, enriched with terms to represent cryptographic messages. Then indistinguishability can be modeled through various behavioral equivalences. In contrast, secrecy or authentication are typically expressed by requesting that some bad event never occurs or that some event (e.g., Bob is logged) is always preceded by another one (e.g., the server granted access to Bob). Then, checking for privacy amounts to checking for trace equivalence between processes, while checking for secrecy amounts to checking that a process never reaches a certain state. Both properties are undecidable in general [33]. Many results have been developed in the context of reachability properties. For an unbounded number of sessions, several decidable classes have been identified (e.g., bounding the size of messages [33] or the number of variables [26]). Tools such as ProVerif [12] or Tamarin [42] do not try to decide security: instead, they propose sound procedures, incomplete but that work well in practice. Tamarin also supports user guidance through lemmas and direct interactions. The case of a bounded number of sessions is known to be (co)-NP-complete [41], and several tools aim at developing efficient decision procedures in practice, such as AVISPA [8] or Scyther [31] (which can also handle an unbounded number of sessions). Results for equivalence properties are more rare. Even in the case of a bounded number of sessions, there are few decidability results, and the associated decision procedures are complex [11, 20,43].

Our Contribution. We consider here a different approach. Instead of trying directly to decide security, we develop a simplification result. Our main contribution reduces the search space for attacks: If there is an attack, then there exists a well-typed attack. More formally, we show that if there is a witness (i.e., a trace) that $P \not Q$, then there exists a witness that is well-typed w.r.t. $P$ or $Q$. Similarly for reachability, for any trace of $P$, we show that there is a well-typed trace of $P$ that follows the same sequence of inputs and outputs, on the same channels, hence preserving secrecy or authentication violations. We can consider arbitrary processes and a family of equational theories that can express most standard primitives: randomized and deterministic asymmetric and symmetric encryptions, signatures, hash, MACs, and even some form of threshold encryption (1 out of $n$ keys or $n$ out of $n$ ). Our class of equational theories mimics the standard equations used, e.g., for encryption and signatures, so any variant can be considered. It still has various restrictions (e.g., at most one non-linear variable) that excludes several theories of interest such as exclusive or. We provide various counter-examples to our simplification result when the equational theory deviates from our assumptions. Our results hold for any typing system provided that any two unifiable encrypted subterms of $P$ ( or $Q$ ) are of the same type. It is then up to the user to adjust the typing system such that this hypothesis holds for the protocols under consideration. The finer the typing system is, the more our typing result restricts the attack search. One way to enforce our assumption is to consider the class of tagged protocols introduced by Blanchet and Podelski [15]. An easy way to achieve this in practice is by labeling encryption and is actually a good protocol design principle $[2,34]$. Our small attack result holds for a notion of equivalence called trace equivalence with static inclusion. Intuitively, $P$ and $Q$ are in equivalence if any trace of $P$ can be matched with a trace of $Q$ such that any equality in $P$ holds in $Q$ (but disequalities in $P$ are not considered), and conversely. Trace equivalence with static inclusion coincides with trace equivalence with static equivalence, as well as observational equivalence [21] for determinate processes; that is, processes such that a sequence of actions may only yield one sequence of message, up to static equivalence.

We extend here a preliminary result presented at Concur'14 [23]. Compared to Reference [23], we considerably enrich the class of cryptographic primitives, since Reference [23] considers (deterministic) symmetric encryption only. Moreover, we provide a small attack property for equivalence as well as reachability properties, while Reference [23] focuses on equivalence. The reachability 
case is somehow an intermediary step of the equivalence case. Therefore, the reachability case was in some sense contained in Reference [23] but not formally stated as an independent result. The simplification result of Reference [23], which we extend, has already been used in several contexts.

- First, in Reference [23] itself, the small attack property is shown to imply decidability for an unbounded number of sessions but for simple protocols (a syntactic subclass of determinate protocols) with no fresh nonces nor fresh keys. Such a decidability result should probably extend to our novel class of primitives, but we chose to focus here on the small attack property.

- Reference [24] establishes the first decidability result for equivalence of protocols with fresh nonces and keys. It uses as a preliminary that only well-typed traces need to be considered.

- SAT-Equiv [28] is a new and efficient tool for deciding trace equivalence for a bounded number of sessions. Thanks to the small attack property, trace equivalence is reduced to finite model-checking and SAT-Equiv adapts standard model-checking techniques, namely graph planning.

Of course, due to the limitations of Reference [23], these three results hold for protocols with symmetric key only. Our extension of the small attack property to a general class of primitives opens the way to new decidability results or more efficient procedures for more general primitives.

Related Work. The analysis of security protocols using formal methods was very successful in discovering new attacks and providing better guarantees. It led to a large number of decision procedures and tools (e.g., References [27, 39, 40]) that have been proposed. Most of these results focus on reachability properties such as confidentiality or authentication. Much fewer results exist for behavioral equivalences. Based on a procedure proposed by Baudet [11], a first decidability result has been proposed for simple processes without else branches, and for equational theories that capture most standard primitives [21]. Then Tiu and Dawson [43] designed and implemented a procedure for open bisimulation, a notion of equivalence stronger than the standard notion of trace equivalence. Cheval et al. [20] have proposed and implemented a procedure for processes with else branches and standard primitives. The tool AkisS [19] is also dedicated to trace equivalence but is not guaranteed to terminate. All these results focus on a bounded number of sessions. The tools ProVerif [14] and Tamarin [42] can handle equivalence properties for an unbounded number of sessions. They actually reason on a stronger notion of equivalence (which may turn out to be too strong in practice) and are not guaranteed to terminate. For an unbounded number of sessions, a few results have been established [22-24].

Our goal here is not to decide equivalence or reachability properties but to restrict the search space for attacks. As already discussed, our result extends the preliminary result of Reference [23] to a wide class of cryptographic primitives and to reachability properties. In terms of proof techniques, we completely reshaped the proof. Reference [23] relies on the fact that reachability is decidable for a bounded number of sessions, builds a decision algorithm for equivalence for a bounded number of sessions, and then uses this algorithm to show that, from any witness of nonequivalence, it is possible to construct a well-typed witness. Our proof here directly builds a welltyped witness, without the need of decision algorithms. Moreover, the fact that Reference [23] considers symmetric key only simplifies the proof, as an attacker never needs to construct when it tries to learn new information. This is no longer true for example with asymmetric encryption or hashes where the attacker may need to encrypt and hash to compare values.

Our proof technique is inspired from the approach developed by Arapinis et al. [7] for bounding the size of messages of an attack for the reachability case. Specifically, they show for some class of tagged protocols that whenever there is an attack, there is a well-typed attack (for a particular typing system). We extend their approach to trace equivalence for slightly more general typing 
systems and more general cryptographic primitives. Indeed, we consider a class of primitives that encompasses all the standard ones while Reference [7] considers a fixed set of primitives (e.g., without randomized encryption). Regarding type systems, we can for example allow type systems that are not structure-preserving. One of the first small model properties has been established by Lowe [38]. It shows that it is sufficient to consider a finite number of roles, but assumes that messages are strongly typed: Agents expect messages that follow a given (fixed) format and may never accept, e.g., an arbitrary message instead of a nonce. Heather et al. [35] provide a result for limiting attacks to well-typed ones, assuming a strong labeling scheme and no blind copies. Their seminal work only includes very basic types such as nonces, agents, or keys and does not consider composed keys. Ramanujam and Suresh [40] also show some kind of typing result, assuming an even stronger labeling scheme, with fresh nonces, and use it to establish a decidability result for protocols with nonces, with no blind copies and the standard primitives. Again for reachability, Mödersheim et al. $[4,36]$ establish a typing result similar to our result for a flexible class of primitives (but incomparable to ours). The type-compliance notion is more restrictive, since even pairs of terms of a protocol should be non-unifiable (or have the same type).

\section{MODEL}

\subsection{Term Algebra}

Private data are represented through an infinite set of names $\mathcal{N}$. Names can model, e.g., long-term and short-term keys, or nonces. We consider an infinite set $\Sigma_{0}^{-}$of constants to represent public data, or any data known by the attacker, such as agent names or attacker's nonces or keys. We also consider two additional infinite sets of constants $\Sigma_{\text {fresh }}^{\text {atom }}$, and $\Sigma_{\text {fresh }}^{\text {bitsting }}$ that we will rely on for our technical development. We write $\Sigma_{\text {fresh }}=\Sigma_{\text {fresh }}^{\text {atom }} \uplus \Sigma_{\text {fresh }}^{\text {bitstring }}$, and $\Sigma_{0}^{+}=\Sigma_{0}^{-} \uplus \Sigma_{\text {fresh }}$. Last, we consider two sets of variables $\mathcal{X}$ and $\mathcal{W}$. Variables in $\mathcal{X}$ typically model arbitrary data expected by the protocol, while variables in $\mathcal{W}$ are used to store messages learned by the attacker. All these sets are assumed to be pairwise disjoint. A data is either a constant, a variable, or a name.

We model messages exchanged on the network and computations of the attacker by terms. A signature $\Sigma$ is a set of function symbols with their arity. We distinguish between constructor symbols, like encryption, in $\Sigma_{\mathrm{c}}$ and destructor symbols, like decryption, in $\Sigma_{\mathrm{d}}$, i.e., $\Sigma=\Sigma_{\mathrm{c}} \uplus \Sigma_{\mathrm{d}}$. Given a signature $\mathcal{F}$, the set of terms built from $\mathcal{F}$ and a set of data $D$ is denoted $\mathcal{T}(\mathcal{F}, D)$. Constructor terms on $D$ are terms in $\mathcal{T}\left(\Sigma_{\mathrm{c}}, D\right)$. We use the usual terminology on terms, which we recall here. We denote $\operatorname{vars}(u)$ the set of variables that occurs in a term $u$. A term is ground if it contains no variable. The application of a substitution $\sigma$ to a term $u$ is written $u \sigma$. We denote $\operatorname{dom}(\sigma)$ its $d o^{-}$ main and $\operatorname{img}(\sigma)$ its image. The positions of a term are defined as usual. Given a term $t$, we denote $\operatorname{root}(t)$ the function symbol occurring at position $\epsilon$ in $t$, and $S t(t)$ its set of subterms. Two terms $u_{1}$ and $u_{2}$ are unifiable when there exists a substitution $\sigma$ such that $u_{1} \sigma=u_{2} \sigma$.

We consider two sorts: atom and bitstring. atom represents atomic data such as nonces or keys, while bitstring models arbitrary messages. Names in $\mathcal{N}$ and constants in $\Sigma_{\text {fresh }}^{\text {atom have sort }}$ atom, whereas constants in $\Sigma_{\text {fresh }}^{\text {bitstring }}$ have sort bitstring. The set $\Sigma_{0}^{-}$contains an infinite number of constants of both sorts. The constants of sort atom are called atomic constants. Any constructor $\mathrm{f}$ comes with its sort, i.e., $\mathrm{f}:\left(s_{1} \times \cdots \times s_{n}\right) \rightarrow s_{0}$ where $n$ is the arity of $\mathrm{f}, s_{0}=$ bitstring, and $s_{i} \in\{$ atom; bitstring $\}$ for $0 \leq i \leq n$. Given a constructor term $t \in \mathcal{T}\left(\Sigma_{\mathrm{c}}, \Sigma_{0}^{+} \uplus \mathcal{X}\right), p$ is an atomic position of $t$ if it corresponds to a position where an atom is expected; that is,

$$
p=p^{\prime} . i,\left.\quad t\right|_{p^{\prime}}=\mathrm{f}\left(t_{1}, \ldots, t_{n}\right) \text { for some } \mathrm{f} \in \Sigma_{\mathrm{c}}:\left(s_{1} \times \cdots \times s_{n}\right) \rightarrow s_{0}, \text { and } s_{i}=\text { atom. }
$$

We say that $t$ is well-sorted if any of its subterm is of the right sort; that is, $\left.t\right|_{p} \in \Sigma_{0}^{-} \uplus \Sigma_{\text {fresh }}^{\text {atom }} \uplus \mathcal{X}$ for any atomic position $p$ of $t$. 
Example 2.1. Randomized asymmetric encryption, pairs, and triples are typically modelled by the following signature:

$$
\Sigma^{\mathrm{ex}}=\left\{\text { raenc, pub, radec, }\langle\rangle,\langle\rangle^{3}, \text { fst, snd, } \operatorname{proj}_{1}^{3}, \operatorname{proj}_{2}^{3}, \operatorname{proj}_{3}^{3}\right\},
$$

with $\Sigma_{\mathrm{c}}^{\mathrm{ex}}=\left\{\right.$ raenc, pub, \langle\rangle$\left.,\langle\rangle^{3}\right\}$, and $\Sigma_{\mathrm{d}}^{\mathrm{ex}}=\left\{\right.$ radec, fst, snd, $\left.\operatorname{proj}_{1}^{3}, \operatorname{proj}_{2}^{3}, \operatorname{proj}_{3}^{3}\right\}$.

The symbols raenc (arity 3 ) and radec (arity 2) represent resp. randomized asymmetric encryption and decryption. The symbol pub is a key function that models the public key associated to a given private key. Pairing is modeled using \langle\rangle of arity 2 , whereas associated projection functions are denoted fst and snd (both of arity 1 ). We also model triples by \langle\rangle$^{3}$ of arity 3 . Projection functions $\operatorname{proj}_{i}^{3}(i \in\{1 ; 2 ; 3\})$ are of arity 1 and retrieve the component $x_{i}$ of the triple $\left\langle x_{1}, x_{2}, x_{3}\right\rangle^{3}$. The sort of our constructors are as follows:

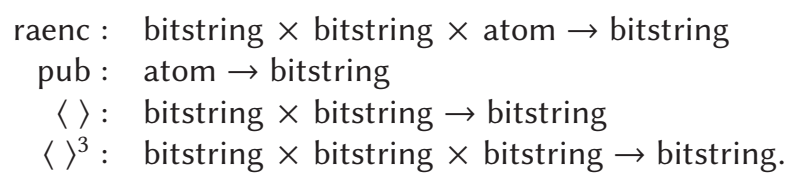

Let $a, b \in \Sigma_{0}^{-}$and $s k_{0}, r_{0} \in \mathcal{N}$ (all of sort atom), the term $u_{0}=\operatorname{raenc}\left(\langle a, b\rangle, \operatorname{pub}(s k), r_{0}\right)$ represents the pair $\langle a, b\rangle$ encrypted by the public key pub(sk) using randomness $r_{0}$. The atomic positions of $u_{0}$ are $p_{1}=2.1$ and $p_{2}=3$. We chose to model randomness as an atom to reflect that randoms are typically non-composed messages. However, we could consider the function symbol raenc with sort: bitstring $\times$ bitstring $\times$ bitstring $\rightarrow$ bitstring as well. In such a situation, $p_{1}=2.1$ would be the only atomic position of $u_{0}$.

We consider theories where, intuitively, each symbol corresponds to a particular function that may be applied only in one particular context. For example, if asymmetric encryption is represented by aenc $(m, \operatorname{pk}(k))$, then it should not be applied to other keys, such as $\operatorname{vk}(k)$. We define as a shape the expected pattern of a message. Intuitively, when a term has a right shape then the application of a rewrite rule may fail only because of disequalities (e.g., attempting to decrypt with the wrong key), but not because of a mismatch between the term and the form of the rewrite rule. A shape is typically more precise than the sort of head symbol. Formally, to each constructor function symbol $\mathrm{f}$, we associate a linear term $\mathrm{f}\left(u_{1}, \ldots, u_{n}\right) \in \mathcal{T}\left(\Sigma_{\mathrm{c}}, \mathcal{X}\right)$ denoted $s h_{\mathrm{f}}$, which is called the shape of $\mathrm{f}$. Shapes have to be compatible; that is, fixed for a given function symbol. Formally, for any $\mathrm{f}\left(t_{1}, \ldots, t_{n}\right)$ occurring in a shape, we have that $\mathrm{f}\left(t_{1}, \ldots, t_{n}\right)=s h_{\mathrm{f}}$. A term is well-shaped if it complies with the shapes; that is, any subterm of $t$, heading with a constructor symbol $\mathrm{f}$ is an instance of the shape of $\mathrm{f}$. More formally, a constructor term $t \in \mathcal{T}\left(\Sigma_{\mathrm{c}}, \Sigma_{0}^{+} \uplus \mathcal{X}\right)$ is well-shaped if for any $t^{\prime} \in S t(t)$ such that $\operatorname{root}\left(t^{\prime}\right)=\mathrm{f}$, we have that $t^{\prime}=s h_{\mathrm{f}} \sigma$ for some substitution $\sigma$. In particular, this notion of compatibility will ensure the existence of well-shaped terms. Given $D \subseteq \Sigma_{0}^{+} \uplus \mathcal{X}$, we denote $\mathcal{T}_{0}\left(\Sigma_{\mathrm{c}}, D\right)$ the subset of ground constructor terms on $D$ that are well-shaped and well-sorted. Given a set $\Sigma_{0}$ of constants (typically $\Sigma_{0}^{-}$or $\left.\Sigma_{0}^{+}\right), \Sigma_{0}$-messages are terms in $\mathcal{T}_{0}\left(\Sigma_{\mathrm{c}}, \mathcal{N} \uplus \Sigma_{0}\right)$.

Example 2.2. Continuing Example 2.1, the shapes associated to each constructor are as follows:

$$
\begin{array}{ll}
\text { raenc }: & s h_{\text {raenc }}=\operatorname{raenc}\left(x_{1}, \operatorname{pub}\left(x_{2}\right), x_{3}\right), \\
\text { pub: } & s h_{\text {pub }}=\operatorname{pub}\left(x_{2}\right), \\
\langle\rangle: & s h_{\langle\rangle}=\left\langle x_{1}, x_{2}\right\rangle, \\
\langle\rangle^{3}: & s h_{\langle\rangle^{3}}=\left\langle x_{1}, x_{2}, x_{3}\right\rangle^{3} .
\end{array}
$$

The term $u_{0}=\operatorname{raenc}\left(\langle a, b\rangle, \operatorname{pub}(s k), r_{0}\right)$ is a constructor term. Actually, this is a $\Sigma_{0}^{-}$-message. We may note that it is well-sorted, since $s k$ and $r_{0}$ are of sort atom, and well-shaped. The constructor term raenc $\left(a, s k, r_{0}\right)$ is well-sorted but it is not well-shaped. Note that we will require protocols to only process messages, which enforces that terms are well-sorted and well-shaped. 
The properties of cryptographic primitives are represented through a set $\mathcal{R}$ of rewriting rules. We consider rewriting rules that apply a destructor on top of constructor terms that are linear, well-sorted, and well-shaped. We strictly control the non-linearity of our rules, and we assume the standard subterm property. More formally, for each destructor symbol des $\in \Sigma_{\mathrm{d}}$, there is exactly one rule of the form $\ell_{\text {des }} \rightarrow r_{\text {des }}$ such that:

(1) $\ell_{\text {des }}=\operatorname{des}\left(t_{1}, \ldots, t_{n}\right)$ where each $t_{i}$ is either a variable or equal to $s h_{\text {root }\left(t_{i}\right)}$ up to a bijective renaming of variables;

(2) $r_{\text {des }} \in \mathcal{T}_{0}\left(\Sigma_{\mathrm{c}}, \emptyset\right) \cup S t\left(t_{1}\right)$. In case $r_{\text {des }}$ is not a fixed constructor term, for simplicity and readability in our technical developments, we assume that $r_{\text {des }}$ occurs in $t_{1}$. Of course, our results easily extend to the case where the arguments of a destructor symbol are written in a different order;

(3) either $\ell_{\text {des }}$ is a linear term or there is a unique variable $x$ with several occurrences in $\ell_{\text {des }}$ and such that $x$ occurs exactly once at an atomic position in $t_{1}$.

Moreover, we assume the existence of at least one non-linear rule in our set $\mathcal{R}$ of rewriting rules.

Given a set $\mathcal{R}$ of rewriting rules, a term $u$ can be rewritten in $v$ using $\mathcal{R}$ if there is a position $p$ in $u$, and a rewriting rule $\mathrm{g}\left(t_{1}, \ldots, t_{n}\right) \rightarrow t$ in $\mathcal{R}$ such that $\left.u\right|_{p}=\mathrm{g}\left(t_{1}, \ldots, t_{n}\right) \theta$ for some substitution $\theta$, and $v=u[t \theta]_{p}$, i.e., $u$ in which the subterm at position $p$ has been replaced by $t \theta$. Moreover, we assume that $t_{1} \theta, \ldots, t_{n} \theta$ as well as $t \theta$ are $\Sigma_{0}^{+}$-messages; in particular, they do not contain destructor symbols. We consider sets of rewriting rules that yield a convergent rewriting system. As usual, we denote $\rightarrow^{*}$ the reflexive-transitive closure of $\rightarrow$, and $u \downarrow$ the normal form of a term $u$ (it is welldefined, as our rewriting system is convergent by unicity of the rule associated to each destructor).

Example 2.3. The properties of the primitives given in Example 2.1 are reflected through the following rewriting rules:

$$
\begin{aligned}
& \mathrm{fst}(\langle x, y\rangle) \rightarrow x, \quad \operatorname{radec}(\operatorname{raenc}(x, \operatorname{pub}(y), z), y) \rightarrow x, \\
& \operatorname{snd}(\langle x, y\rangle) \rightarrow y, \quad \operatorname{proj}_{i}^{3}\left(\left\langle x_{1}, x_{2}, x_{3}\right\rangle_{3}\right) \rightarrow x_{i} \quad \text { with } i \in\{1 ; 2 ; 3\} .
\end{aligned}
$$

They satisfy all the requirements stated above.

Our class of rewriting rules is flexible enough to represent most of the standard primitives as illustrated in the following example. However, we cannot model, for instance, a decryption algorithm that never fails and always returns a bitstring (e.g., $\operatorname{sdec}(m, k)$ ). Indeed, such a term is not a message and will not be accepted as input or output of a protocol. We cannot model either any primitive that includes an associative and commutative operator, such as the exclusive or, or the modular exponentiation.

Example 2.4. We may consider symmetric encryption (randomized or not) using the signature $\Sigma^{\text {senc }}=\{$ senc, rsenc, sdec, $r$ sdec $\}$ and the rewriting rules:

$$
\begin{array}{rlr}
\text { senc : } & \text { bitstring } \times \text { atom } \rightarrow \text { bitstring, } & s h_{\mathrm{rsenc}}=\operatorname{senc}\left(x_{1}, x_{2}\right), \\
\text { rsenc : } & \text { bitstring } \times \text { atom } \times \text { atom } \rightarrow \text { bitstring, } \quad & \operatorname{sh} h_{\mathrm{rsenc}}=\operatorname{rsenc}\left(x_{1}, x_{2}, x_{3}\right), \\
& \operatorname{sdec}(\operatorname{senc}(x, y), y) \rightarrow x, \quad \operatorname{rddec}(\operatorname{rsenc}(x, y, z), y) \rightarrow x .
\end{array}
$$

Of course, we may consider non-randomized asymmetric encryption as well. We can also model signature and hash function through the signature $\Sigma^{\text {sign }}=\{$ sign, getmsg, check, vk, ok, hash $\}$ :

$$
\begin{aligned}
& \text { ok: } \rightarrow \text { bitstring, } \\
& \text { sign : bitstring } \times \text { atom } \rightarrow \text { bitstring, } \quad s h_{\text {sign }}=\operatorname{sign}\left(x_{1}, x_{2}\right) \text {, } \\
& \mathrm{vk}: \text { atom } \rightarrow \text { bitstring, } \quad s h_{\mathrm{vk}}=\mathrm{vk}\left(x_{1}\right) \text {, } \\
& \text { hash : bitstring } \rightarrow \text { bitstring, } \quad s h_{\text {hash }}=\operatorname{hash}\left(x_{1}\right) \text {, } \\
& \operatorname{getmsg}(\operatorname{sign}(x, y)) \rightarrow x, \quad \operatorname{check}(\operatorname{sign}(x, y), \operatorname{vk}(y)) \rightarrow \text { ok. }
\end{aligned}
$$


We may also represent more exotic theory such as 1 out of $n$ encryption (that is, one key among $n$ suffices to decrypt) and $n$ out of $n$ encryption (that is, the $n$ shares of the key are needed to decrypt)

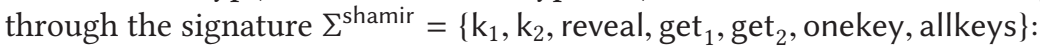

$$
\begin{array}{rlrl}
\mathrm{k}_{i}: & \text { atom } \rightarrow \text { bitstring, } & & s h_{\mathrm{k}_{i}}=\mathrm{k}_{i}\left(x_{1}\right) ; \\
\text { onekey : } & \text { bitstring } \times \text { atom } \times \text { atom, } & s h_{\text {onekey }}=\text { onekey }\left(x_{1}, x_{2}, x_{3}\right) ; \\
\text { allkeys : } & \text { bitstring } \times \text { atom, } & & s h_{\text {allkeys }}=\text { allkeys }\left(x_{1}, x_{2}\right) ; \\
\text { get }_{i}\left(\text { onekey }\left(x, y_{1}, y_{2}\right), y_{i}\right) \rightarrow x \text { with } i \in\{1,2\}, & \text { reveal (allkeys } \left.(x, y), \mathrm{k}_{1}(y), \mathrm{k}_{2}(y)\right) \rightarrow x .
\end{array}
$$

We can also model tuples of various size in a similar fashion than triples in Example 2.1. Another theory of interest is when it is possible to check whether two ciphertexts have been encrypted with the same key. This can be modeled by adding the destructor symbol samekey to $\Sigma^{\text {senc }}$ and the rewrite rule

$$
\operatorname{samekey}\left(\operatorname{senc}\left(x_{1}, y\right), \operatorname{senc}\left(x_{2}, y\right)\right) \rightarrow \text { ok }
$$

Note, however, that the theory for equality, often modeled by the simple rule eq $(x, x) \rightarrow$ ok does not fit our assumptions. First, since $x$ is a non-linear variable, it needs to be of sort atom, which seriously limits test for equalities. But even with this restriction, the rewrite rule cannot be accepted; Since $x$ appears directly under a destructor, there is no constructor associated to it.

An attacker builds his own messages by applying public function symbols to terms he already knows and that are available through variables in $\mathcal{W}$. Formally, given a set $\Sigma_{0}$ of constants (typically $\Sigma_{0}^{-}$or $\left.\Sigma_{0}^{+}\right)$, a computation done by the attacker is a $\Sigma_{0}$-recipe, i.e., a term in $\mathcal{T}\left(\Sigma, \mathcal{W} \uplus \Sigma_{0}\right)$.

\subsection{Process Algebra}

Our process algebra is inspired from the applied pi calculus [1]. We do not consider else branches. Actually, we do not have conditional. Instead, equality tests are performed through patternmatching. We do not consider replication, but our typing result easily extends to processes with replication, as explained in Section 6 (see also Reference [23]). Indeed, our key result shows how to build a well-typed trace from an arbitrary one. This holds for traces obtained from finite processes as well as traces from replicated processes.

Let $C h$ be an infinite set of channels. We consider processes built using the following grammar:

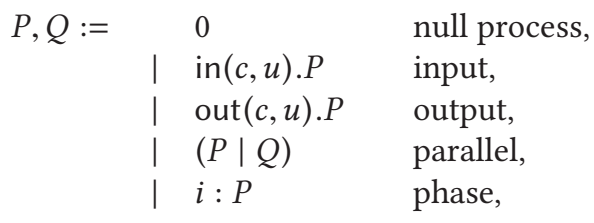

where $u \in \mathcal{T}_{0}\left(\Sigma_{\mathrm{c}}, \Sigma_{0}^{-} \uplus \mathcal{N} \uplus \mathcal{X}\right)$, and $c \in C h$.

The process 0 does nothing. The process in $(c, u) . P$ expects a message $m$ of the form $u$ on channel $c$ and then behaves like $P \sigma$ where $\sigma$ is a substitution such that $m=u \sigma$. The process out $(c, u) . P$ emits $u$ on channel $c$, and then behaves like $P$. The variables that occur in $u$ are instantiated when the evaluation takes place. The process $P \mid Q$ runs $P$ and $Q$ in parallel. Our calculus also introduces a phase instruction, in the spirit of Reference [14], denoted $i: P$.

For the sake of clarity, we may omit the null process. We write $f v(P)$ for the set of free variables that occur in $P$, i.e., the set of variables that is not in the scope of an input. We also assume that processes are variable distinct, i.e., any variable is at most bound once. For example, in the process in $(c, x)$.in $(c, x)$ the variable $x$ is bound once, whereas in the process in $(c, x) \mid \operatorname{in}(c, x)$, one occurrence of the variable $x$ should be renamed to ensure variable distinctness. 
Phases are useful to specify properties inspired from cryptographic, game-based security definitions. A typical example is the real-or-random property: After interacting with a protocol, an attacker is given the real secret or a random one and she should not make the difference. Phases are also used to state unlinkability properties [10] to model that an attacker should not be able to distinguish between Alice and Bob, even after having interacted with Alice. Some protocols also directly include several steps that are best modeled with phases. This is, for example, the case of voting protocols [16].

Example 2.5. We consider a variant of the Needham Schroeder Lowe public key protocol [37] with randomized encryption. The protocol aims at ensuring mutual authentication through the secrecy of the nonces $N_{a}$ and $N_{b}$ that are exchanged during an execution. It can be described informally as follows:

$$
\begin{array}{ll}
\text { 1. } A \rightarrow B: & \operatorname{raenc}\left(\left\langle A, N_{a}\right\rangle, \operatorname{pub}(B), r_{1}\right), \\
\text { 2. } B \rightarrow A: & \operatorname{raenc}\left(\left\langle N_{a},\left\langle N_{b}, B\right\rangle\right\rangle, \operatorname{pub}(A), r_{2}\right), \\
\text { 3. } A \rightarrow B: & \operatorname{raenc}\left(N_{b}, \operatorname{pub}(B), r_{3}\right),
\end{array}
$$

where $A$ and $B$ are agents trying to authenticate each other, pub $(A)$ (resp. pub $(B)$ ) is the public key of $A$ (resp. $B$ ), $N_{a}$ and $N_{b}$ (as well as $r_{1}, r_{2}$, and $r_{3}$ ) are nonces generated by $A$ and $B$. This is a slight variant of the original protocol [37] proposed by J. Millen: In the second message, the identity of $B$ is placed at the end of the message, instead of the beginning of the message. This variant is subject to a type-flaw attack (discovered by J. Millen), as we shall explain in the next section.

We model the Needham Schroeder Lowe protocol in our formalism through the process $P_{\mathrm{NSL}}$, which results from the composition of the process $P_{A}$ representing the role of $A$ and the process $P_{B}$ representing the role of $B$.

$$
P_{\mathrm{NSL}}=P_{A} \mid P_{B},
$$

with $P_{A}$ and $P_{B}$ defined as follows.

$$
\begin{array}{rlrl}
P_{A}= & \operatorname{out}\left(c_{A}, \operatorname{raenc}\left(\left\langle\mathrm{a}, n_{a}\right\rangle, \operatorname{pub}\left(s k_{b}\right), r_{1}\right) . \quad P_{B}=\right. & \operatorname{in}\left(c_{B}, \operatorname{raenc}\left(\left\langle\mathrm{a}, y_{1}\right\rangle, \operatorname{pub}\left(s k_{b}\right), y_{2}\right)\right) . \\
& \operatorname{in}\left(c_{A}, \operatorname{raenc}\left(\left\langle n_{a},\left\langle x_{1}, \mathrm{~b}\right\rangle\right\rangle, \operatorname{pub}\left(s k_{a}\right), x_{2}\right)\right) . & & \operatorname{out}\left(c_{B}, \operatorname{raenc}\left(\left\langle y_{1},\left\langle n_{b}, \mathrm{~b}\right\rangle\right\rangle, \operatorname{pub}\left(s k_{a}\right), r_{2}\right)\right) . \\
& \operatorname{out}\left(c_{A}, \operatorname{raenc}\left(x_{1}, \operatorname{pub}\left(s k_{b}\right), r_{3}\right)\right) . & & \operatorname{in}\left(c_{B}, \operatorname{raenc}\left(n_{b}, \operatorname{pub}\left(s k_{b}\right), y_{3}\right)\right),
\end{array}
$$

where $s k_{a}, s k_{b}, n_{a}, n_{b}, r_{1}, r_{2}$, and $r_{3}$ are names, whereas a and $\mathrm{b}$ are constants from $\Sigma_{0}^{-}$.

To model a richer scenario, we may want to consider in addition the process $P_{B}^{\prime}$ that corresponds to the role $B$ played by agent a interacting with a dishonest agent c. Below, $r_{3}^{\prime}, n_{b}^{\prime} \in \mathcal{N}$ whereas c, $s k_{c} \in \Sigma_{0}^{-}$(so they are implicitly given to the attacker). We write $\bar{P}_{\mathrm{NSL}}=P_{B} \mid P_{B}^{\prime}$

$$
\begin{aligned}
P_{B}^{\prime}= & \operatorname{in}\left(c_{B}^{\prime}, \operatorname{raenc}\left(\left\langle c, y_{1}^{\prime}\right\rangle, \operatorname{pub}\left(s k_{a}\right), y_{2}^{\prime}\right)\right) . \\
& \operatorname{out}\left(c_{B}^{\prime}, \operatorname{raenc}\left(\left\langle y_{1}^{\prime},\left\langle n_{b}^{\prime}, \mathrm{a}\right\rangle\right\rangle, \operatorname{pub}\left(s k_{c}\right), r_{2}^{\prime}\right)\right) . \\
& \operatorname{in}\left(c_{B}^{\prime}, \operatorname{raenc}\left(n_{b}^{\prime}, \operatorname{pub}\left(s k_{a}\right), y_{3}^{\prime}\right)\right) .
\end{aligned}
$$

\subsection{Semantics}

The operational semantics of a process is defined using a relation over configurations. Configurations are parameterized by a set of constants $\Sigma_{0}$ (typically $\Sigma_{0}^{-}$or $\Sigma_{0}^{+}$). A $\Sigma_{0}$-configuration is a tuple $(\mathcal{P} ; \phi ; \sigma ; i)$ with $i \in \mathbb{N}$ such that:

- $\mathcal{P}$ is a multiset of processes (not necessarily ground);

- $\phi=\left\{\mathrm{w}_{1} \triangleright m_{1}, \ldots, \mathrm{w}_{n} \triangleright m_{n}\right\}$ is a $\Sigma_{0}$-frame, i.e., a substitution where $\mathrm{w}_{1}, \ldots, \mathrm{w}_{n}$ are variables in $\mathcal{W}$, and $m_{1}, \ldots, m_{n}$ are $\Sigma_{0}$-messages;

- $\sigma$ is a substitution such that $\operatorname{dom}(\sigma)=f v(\mathcal{P})$, and $\operatorname{img}(\sigma)$ are $\Sigma_{0}$-messages.

A $\Sigma_{0}$-configuration $(\mathcal{P} ; \phi ; \sigma ; i)$ such that $\sigma=\emptyset$ is said initial. 


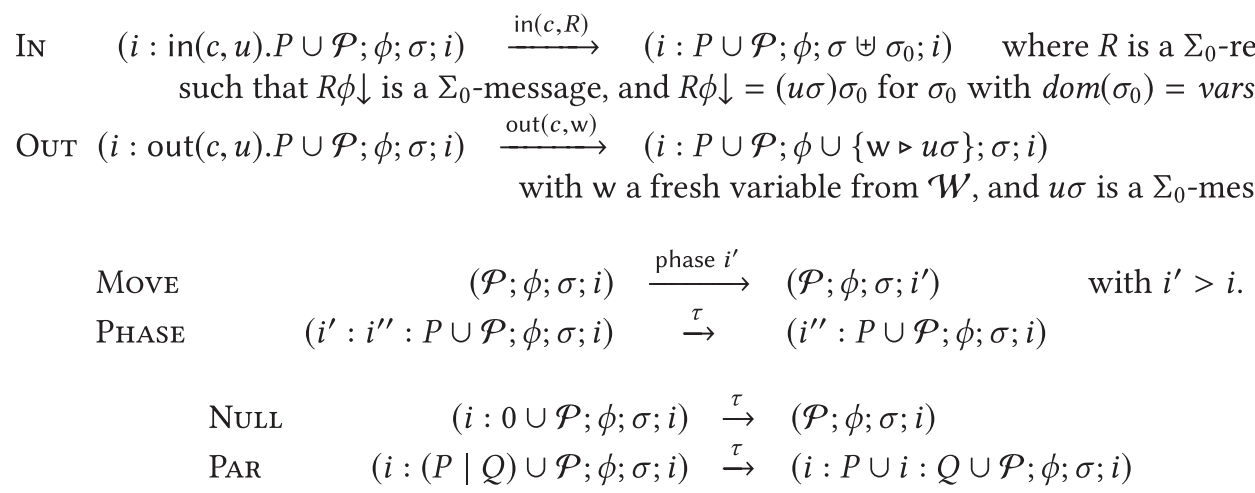

Fig. 1. Semantics for processes w.r.t. $\Sigma_{0}$.

Intuitively, $\mathcal{P}$ represents the processes that still remain to be executed; $\phi$ represents the sequence of messages that have been learned so far by the attacker, $\sigma$ stores the value of the variables that have already been instantiated, and $i$ is an integer that indicates the current phase. We often write $i$ : $P$ instead of $(P ; \emptyset ; \emptyset ; i), P$ instead of $0: P$, and $P \uplus \mathcal{P}$ instead of $\{P\} \uplus \mathcal{P}$. The operational semantics of a $\Sigma_{0}$-configuration is induced by the relation $\stackrel{\alpha}{\rightarrow}$ w.r.t. $\Sigma_{0}$ over $\Sigma_{0}$-configurations defined in Figure 1 .

The first rule (IN) allows the attacker to send to some process a term built from publicly available terms and symbols. The second rule (Out) corresponds to the output of a term by some process: The corresponding term is added to the frame of the current configuration, which means that the attacker can now access the sent term. Note that the term is output provided that it is a message. Regarding phases (rules Move and PHASE), the attacker may move to a subsequent phase whenever he wants, while processes may move to the next phase when they are done. The two remaining rules NULL and PAR are quite standard and are unobservable ( $\tau$ action) from the point of view of the attacker.

The relation $\stackrel{\alpha_{1} \ldots \alpha_{n}}{\longrightarrow}$ w.r.t. $\Sigma_{0}$ between $\Sigma_{0}$-configurations (where $\alpha_{1} \ldots \alpha_{n}$ is a sequence of actions) is defined as the transitive closure of $\stackrel{\alpha}{\rightarrow}$ w.r.t. $\Sigma_{0}$. Given a sequence of observable actions $t$, and two $\Sigma_{0}$-configurations $\mathcal{K}$ and $\mathcal{K}^{\prime}$, we write $\mathcal{K} \stackrel{\text { tr }}{\Longrightarrow} \mathcal{K}^{\prime}$ w.r.t. $\Sigma_{0}$ when there exists a sequence $\alpha_{1} \ldots \alpha_{n}$ such that $\mathcal{K} \stackrel{\alpha_{1} \ldots \alpha_{n}}{\longrightarrow} \mathcal{K}^{\prime}$ w.r.t. $\Sigma_{0}$ and tr is obtained from $\alpha_{1} \ldots \alpha_{n}$ by erasing all occurrences of $\tau$.

Definition 2.6. Given a $\Sigma_{0}$-configuration $\mathcal{K}=(\mathcal{P} ; \phi ; \sigma ; i)$, we denote trace $\Sigma_{\Sigma_{0}}(\mathcal{K})$ the set of traces defined as follows:

$\operatorname{trace}_{\Sigma_{0}}(\mathcal{K})=\left\{\left(\operatorname{tr}, \phi^{\prime}\right) \mid \mathcal{K} \stackrel{\operatorname{tr}}{\Longrightarrow}\left(\mathcal{P} ; \phi^{\prime} ; \sigma^{\prime} ; i^{\prime}\right)\right.$ w.r.t. $\Sigma_{0}$ for some $\Sigma_{0}$-configuration $\left.\left(\mathcal{P} ; \phi^{\prime} ; \sigma^{\prime} ; i^{\prime}\right)\right\}$.

Given a $\Sigma_{0}$-configuration $\mathcal{K}$, we may note that, by definition of $\operatorname{trace}_{\Sigma_{0}}(\mathcal{K})$, we have that $\operatorname{tr} \phi \downarrow$, i.e., $\operatorname{tr}$ on which we apply the substitution $\phi$ followed by rewriting steps to normalize the second argument of each input/ouput action, only contains $\Sigma_{0}$-messages.

Example 2.7. Continuing Example 2.5, consider the initial configuration $\mathcal{K}_{\mathrm{NSL}}=\left(\bar{P}_{\mathrm{NSL}} ; \phi_{0} ; \emptyset ; 0\right)$ with initial knowledge $\phi_{0}=\left\{w_{a} \triangleright \operatorname{pub}\left(s k_{a}\right), w_{b} \triangleright \operatorname{pub}\left(s k_{b}\right)\right\}$. This models that the attacker initially knows the public keys of a and b. Note that the private key of c, $s k_{c}$, is a public constant and is thus also initially known to the attacker.

As mentioned in Example 2.5, this variant of the Needham Schroeder Lowe protocol is subject to a type flaw attack depicted in Figure 2. The attack relies on the fact that agent $A$ may accept the 


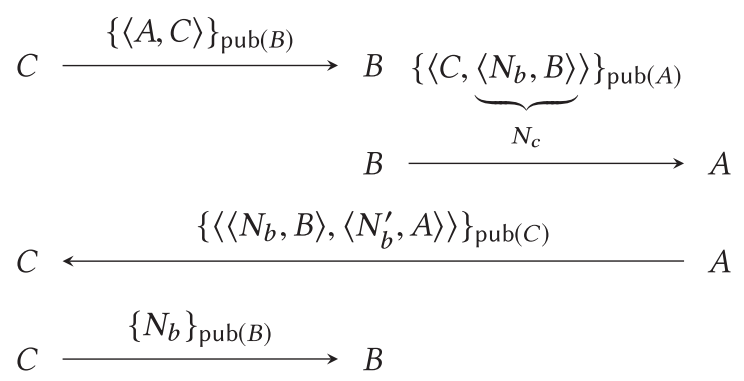

Fig. 2. Attack trace.

pair $\left\langle N_{b}, B\right\rangle$ as a nonce $N_{C}$ coming from $C$, and agent $B$ may confuse the identity $C$ with a nonce. In this attack, a dishonest agent $C$ initiates a session with agent $B$ but writes his identity $C$ instead of a nonce. $B$ replies as expected by $\left\{C, N_{b}, B\right\}_{\text {pub }(A)}$. This message is then accepted by $A$ (playing the responder role). $A$ thinks she is contacted by $C$ and replies with $\left\{N_{b}, B, N_{b}^{\prime}, A\right\}_{\text {pub }(C)}$. Therefore, the attacker learns $N_{b}$ and may impersonate $A$ w.r.t. $B$. This attack is reflected by the following sequence tr:

$$
\operatorname{in}\left(c_{B}, \operatorname{raenc}\left(\langle\mathrm{a}, \mathrm{c}\rangle, \mathrm{w}_{b}, r_{0}\right)\right) \cdot \operatorname{out}\left(c_{B}, \mathrm{w}_{1}\right) \cdot \operatorname{in}\left(c_{B}^{\prime}, \mathrm{w}_{1}\right) \cdot \operatorname{out}\left(c_{B}^{\prime}, \mathrm{w}_{2}\right) \cdot \operatorname{in}\left(c_{B}, \operatorname{raenc}\left(R, \mathrm{w}_{b}, r_{0}^{\prime}\right)\right),
$$

where $R=\mathrm{fst}\left(\operatorname{fst}\left(\operatorname{radec}\left(\mathrm{w}_{2}, s k_{c}\right)\right)\right)$ and $r_{0}, r_{0}^{\prime} \in \Sigma_{0}^{-}$. This sequence of actions yields the frame $\phi$ defined as follows:

$$
\phi=\phi_{0} \uplus\left\{\mathrm{w}_{1} \triangleright \operatorname{raenc}\left(\left\langle\mathrm{c},\left\langle n_{b}, \mathrm{~b}\right\rangle\right\rangle, \operatorname{pub}\left(s k_{a}\right), r_{2}\right), \mathrm{w}_{2} \triangleright \operatorname{raenc}\left(\left\langle\left\langle n_{b}, \mathrm{~b}\right\rangle,\left\langle n_{b}^{\prime}, \mathrm{a}\right\rangle\right\rangle, \operatorname{pub}\left(s k_{c}\right), r_{2}^{\prime}\right)\right\} .
$$

We have that $(\operatorname{tr}, \phi) \in \operatorname{trace}\left(\mathcal{K}_{\mathrm{NSL}}\right)$.

\subsection{Trace Equivalence}

Many privacy properties such as vote-privacy or untraceability are expressed as trace equivalence $[6,32]$. Intuitively, two configurations are trace equivalent if an attacker cannot tell with which of the two configurations he is interacting. We first introduce a notion of equivalence between frames.

Intuitively, an attacker can see the difference between two sequences of messages if he is able to perform some computation that succeeds in $\phi_{1}$ and fails in $\phi_{2}$; or if he can build a test that leads to an equality in $\phi_{1}$ and not in $\phi_{2}$ (or conversely).

Definition 2.8. Two $\Sigma_{0}$-frames $\phi_{1}$ and $\phi_{2}$ are in static inclusion w.r.t. $\Sigma_{0}$, written $\phi_{1} \sqsubseteq_{s} \phi_{2}$ w.r.t. $\Sigma_{0}$, when $\operatorname{dom}\left(\phi_{1}\right)=\operatorname{dom}\left(\phi_{2}\right)$, and:

- for any $\Sigma_{0}$-recipe $R$, we have that $R \phi_{1} \downarrow$ is $\Sigma_{0}$-message implies that $R \phi_{2} \downarrow$ is $\Sigma_{0}$-message; and

- for any $\Sigma_{0}$-recipes $R, R^{\prime}$ such that $R \phi_{1} \downarrow, R^{\prime} \phi_{1} \downarrow$ are $\Sigma_{0}$-messages, we have that: $R \phi_{1} \downarrow=R^{\prime} \phi_{1} \downarrow$ implies $R \phi_{2} \downarrow=R^{\prime} \phi_{2} \downarrow$.

They are in static equivalence w.r.t. $\Sigma_{0}$, written $\phi_{1} \sim_{s} \phi_{2}$ w.r.t. $\Sigma_{0}$, if $\phi_{1} \sqsubseteq_{s} \phi_{2}$ and $\phi_{2} \sqsubseteq_{s} \phi_{1}$.

In the remainder of this article, $\Sigma_{0}$ will be either $\Sigma_{0}^{-}$or $\Sigma_{0}^{+}$, and we sometimes omit to mention it when it is clear from the context.

Example 2.9. Continuing Example 2.7, we consider the two following $\Sigma_{0}^{-}$-frames:

- $\phi_{1}=\phi \uplus\left\{\mathrm{w}_{3} \triangleright n_{b}\right\}$; and

- $\phi_{2}=\phi \uplus\left\{\mathrm{w}_{3} \triangleright k\right\}$. 
We have that $R \phi_{1} \downarrow=\mathrm{w}_{3} \phi_{1} \downarrow$ where $R=\mathrm{fst}\left(\mathrm{fst}\left(\operatorname{radec}\left(\mathrm{w}_{2}, s k_{c}\right)\right)\right)$. This equality does not hold in $\phi_{2}$, hence $\phi_{1}$ and $\phi_{2}$ are not in static equivalence.

Trace equivalence is the active counterpart of static equivalence. Two configurations are traceequivalent if, however the attacker behaves, the resulting sequences of messages observed by the attacker are in static equivalence.

Definition 2.10. A $\Sigma_{0}$-configuration $\mathcal{K}$ is trace included (w.r.t. $\Sigma_{0}$ ) in a $\Sigma_{0}$-configuration $\mathcal{K}^{\prime}$, written $\mathcal{K} \sqsubseteq_{t} \mathcal{K}^{\prime}$ w.r.t. $\Sigma_{0}$, if for every $(\operatorname{tr}, \phi) \in \operatorname{trace}_{\Sigma_{0}}(\mathcal{K})$, there exist $\left(\operatorname{tr}^{\prime}, \phi^{\prime}\right) \in \operatorname{trace}_{\Sigma_{0}}\left(\mathcal{K}^{\prime}\right)$ such that $\operatorname{tr}=\operatorname{tr}^{\prime}$, and $\phi \sqsubseteq_{s} \phi^{\prime}$ w.r.t. $\Sigma_{0}$. They are in trace equivalence, written $\mathcal{K} \approx_{t} \mathcal{K}^{\prime}$ w.r.t. $\Sigma_{0}, \mathcal{K} \sqsubseteq_{t}$ $\mathcal{K}^{\prime}$ and $\mathcal{K}^{\prime} \sqsubseteq_{t} \mathcal{K}$ w.r.t. $\Sigma_{0}$.

Note that two trace-equivalent configurations are necessarily at the same phase.

This notion of trace equivalence slightly differs from the one used in Reference [23], where the frames are required to be in static equivalence $\phi \sim_{s} \phi^{\prime}$ instead of static inclusion $\phi \sqsubseteq_{s} \phi^{\prime}$. Actually, these two notions of equivalence coincide for determinate protocols [19].

Definition 2.11. A configuration $\mathcal{K}$ is determinate if whenever $\mathcal{K} \stackrel{\text { tr }}{\Longrightarrow} \mathcal{K}_{1}$ and $\mathcal{K} \stackrel{\text { tr }}{\Longrightarrow} \mathcal{K}_{2}$ for some tr, $\mathcal{K}_{1}=\left(\mathcal{P}_{1} ; \phi_{1} ; \sigma_{1} ; i_{1}\right)$, and $\mathcal{K}_{2}=\left(\mathcal{P}_{2} ; \phi_{2} ; \sigma_{2} ; i_{2}\right)$. We have that $\phi_{1} \sim_{s} \phi_{2}$.

Determinate protocols encompass the class of simple protocols as given, e.g., in Reference [21]. Intuitively, simple protocols are protocols such that each copy of a replicated process has its own channel reflecting the fact that due to IP addresses and session identifiers, an attacker can identify which process and which session he is sending messages to (or receiving messages from).

Trace equivalence can be used to express various privacy properties. A first example is anonymity: A protocol $P$ preserves Alice's anonymity if an attacker cannot distinguish whether she is interacting with Alice or Bob.

$$
P(\text { Alice }) \approx_{t} P(B o b),
$$

where $P($ Alice $)$ represents a configuration where Alice is present.

Vote privacy is slightly more complicated. An attacker may know whether Alice voted or not. However, she should not be able to distinguish the scenario where Alice votes 0 and Bob votes 1 , from the converse scenario where Alice votes 1 and Bob votes 0 . This can be (informally) expressed through the following equivalence [32]:

$$
\operatorname{Voter}(\text { Alice }, 0) \mid \operatorname{Voter}(B o b, 1) \approx_{t} \operatorname{Voter}(\text { Alice }, 1) \mid \operatorname{Voter}(B o b, 0) .
$$

Stronger privacy properties such as receipt-freeness and coercion resistance can also be modeled using equivalences [32].

Another example is unlinkability in the context of IoT. For example, it should not be possible to trace Alice, even if she is wearing a biometric passport (or another tag). More precisely, even if an attacker observes (and interacts with) many sessions of Alice and other passport holders, she should not be able to distinguish between a session with Alice and a session with Bob. Assume that $P($ Alice,$r)$ models a session of Alice's passport (with a reader) with some fresh random $r$. Then unlinkability can be (informally) expressed as follows [18]:

$$
\begin{aligned}
& P\left(A_{1}, r_{1}^{1}\right)|\cdots| P\left(A_{1}, r_{1}^{n}\right)|\cdots| P\left(A_{k}, r_{k}^{1}\right)\left|\cdots P\left(A_{k}, r_{k}^{n}\right)\right| 1: P\left(A_{1}, r\right) \\
\approx_{t} \quad & P\left(A_{1}, s_{1}^{1}\right)|\cdots| P\left(A_{1}, s_{1}^{n}\right)|\cdots| P\left(A_{k}, s_{k}^{1}\right)\left|\cdots P\left(A_{k}, s_{k}^{n}\right)\right| 1: P\left(A_{2}, s\right) .
\end{aligned}
$$

Finally, nonce or key secrecy can be modeled as a real-or-random game. Once the attacker is done interacting with the protocol $P$ that establishes a secret $s$, then she is given either $s$ or a fresh 
random $r$ and she should try to distinguish between these two scenarios. This is often called strong secrecy.

$$
P(s)\left|1: \operatorname{out}(c, s) \approx_{t} P(s)\right| 1: \text { out }(c, r)
$$

The use of a phase is necessary to avoid false attacks. Indeed, an attacker could then try to reuse the received (real or random) secret and re-inject it in the true protocol. This real-or-random property corresponds to a stronger modeling of secrecy than standard secrecy properties modeled as reachability properties. Secrecy stated as a reachability states that the attacker is not able to compute the secret. Strong secrecy guarantees that the attacker should gain absolutely no information on the secret. For example, publishing the hash of a secret $h(s)$ preserves the secrecy of $s$ but not its strong secrecy.

Example 2.12. Continuing Example 2.5, we consider the protocol $\bar{P}_{\mathrm{NSL}}$ that models two roles of $B$ : one played by $B$ responding to $A$, and the other one played by $A$ responding to $C$. As explained above, to model the fact that the nonce $n_{b}$ sent by $B$ for $A$ should remain secret, we require that $n_{b}$ remains indistinguishable from a fresh value. Formally, we add a new phase to the process $P_{B}$. Once the attacker is done interacting with the standard protocol, then $B$ will output either the true nonce $n_{b}$ or a fresh value $k \in \mathcal{N}$. This yields the following two processes.

$$
\begin{aligned}
& P_{B}^{1}=\operatorname{in}\left(c_{B}, \operatorname{raenc}\left(\left\langle\mathrm{a}, y_{1}\right\rangle, \operatorname{pub}\left(s k_{b}\right), y_{2}\right)\right) . \quad P_{B}^{2}=\operatorname{in}\left(c_{B}, \operatorname{raenc}\left(\left\langle\mathrm{a}, y_{1}\right\rangle, \operatorname{pub}\left(s k_{b}\right), y_{2}\right)\right) \text {. } \\
& \operatorname{out}\left(c_{B}, \operatorname{raenc}\left(\left\langle y_{1},\left\langle n_{b}, \mathrm{~b}\right\rangle\right\rangle, \operatorname{pub}\left(s k_{a}\right), r_{2}\right)\right) . \quad \operatorname{out}\left(c_{B}, \operatorname{raenc}\left(\left\langle y_{1},\left\langle n_{b}, \mathrm{~b}\right\rangle\right\rangle, \operatorname{pub}\left(s k_{a}\right), r_{2}\right)\right) \text {. } \\
& \operatorname{in}\left(c_{B}, \operatorname{raenc}\left(n_{b}, \operatorname{pub}\left(s k_{b}\right), y_{3}\right)\right) \text {. } \operatorname{in}\left(c_{B}, \operatorname{raenc}\left(n_{b}, \operatorname{pub}\left(s k_{b}\right), y_{3}\right)\right) \text {. } \\
& 1: \operatorname{out}\left(c_{B}, n_{b}\right) \quad 1: \operatorname{out}\left(c_{B}, k\right)
\end{aligned}
$$

The corresponding overall processes are $\bar{P}_{\mathrm{NSL}}^{1}=P_{B}^{\prime} \mid P_{B}^{1}$ and $\bar{P}_{\mathrm{NSL}}^{2}=P_{B}^{\prime} \mid P_{B}^{2}$, and we consider the initial frame $\phi_{0}$ as given in Example 2.7 and the initial configurations $\mathcal{K}_{1}=\left(\bar{P}_{\mathrm{NSL}}^{1} ; \phi_{0} ; \emptyset ; 0\right)$ and $\mathcal{K}_{2}=\left(\bar{P}_{\mathrm{NSL}}^{2} ; \phi_{0} ; \emptyset ; 0\right)$. In this example, the use of a phase may not be strictly necessary w.r.t. the existence of attacks. But in principle, removing the phase could yield to false attacks.

We can show that $\mathcal{K}_{1} \nsubseteq_{t} \mathcal{K}_{2}$, since $n_{b}$ is not strongly secret due to the attack depicted in Figure 2 . This is exemplified by the trace

$$
\operatorname{tr}=\operatorname{in}\left(c_{B}, R_{1}\right) \cdot \operatorname{out}\left(c_{B}, \mathrm{w}_{1}\right) \cdot \operatorname{in}\left(c_{B}^{\prime}, \mathrm{w}_{1}\right) \cdot \operatorname{out}\left(c_{B}^{\prime}, \mathrm{w}_{2}\right) \cdot \operatorname{in}\left(c_{B}, R_{2}\right) \cdot \operatorname{out}\left(c_{B}, \mathrm{w}_{3}\right),
$$

with $R_{1}=\operatorname{raenc}\left(\langle\mathrm{a}, \mathrm{c}\rangle, \mathrm{w}_{b}, r_{0}\right)$ and $R_{2}=\operatorname{raenc}\left(\mathrm{fst}\left(\mathrm{fst}\left(\operatorname{radec}\left(\mathrm{w}_{2}, s k_{c}\right)\right)\right), \mathrm{w}_{b}, r_{0}^{\prime}\right)$, where $r_{0}, r_{0}^{\prime} \in \Sigma_{0}^{-}$. Indeed, consider now

$$
\phi=\phi_{0} \uplus\left\{\mathrm{w}_{1} \triangleright \operatorname{raenc}\left(\left\langle\mathrm{c},\left\langle n_{b}, \mathrm{~b}\right\rangle\right\rangle, \operatorname{pub}\left(s k_{a}\right), r_{2}\right), \mathrm{w}_{2} \triangleright \operatorname{raenc}\left(\left\langle\left\langle n_{b}, \mathrm{~b}\right\rangle,\left\langle n_{b}^{\prime}, \mathrm{a}\right\rangle\right\rangle, \operatorname{pub}\left(s k_{c}\right), r_{2}^{\prime}\right)\right\}
$$

and $\phi_{1}=\phi_{0} \uplus\left\{\mathrm{w}_{3} \triangleright n_{b}\right\}, \quad \phi_{2}=\phi \uplus\left\{\mathrm{w}_{3} \triangleright k\right\}$. We have $\left(\operatorname{tr}, \phi_{1}\right) \in \operatorname{trace}_{\Sigma_{0}^{-}}\left(\mathcal{K}_{1}\right)$ and $\left(\operatorname{tr}, \phi_{2}\right) \in$ $\operatorname{trace}_{\Sigma_{0}^{-}}\left(\mathcal{K}_{2}\right)$. Now consider the equality $\mathrm{w}_{3}=\mathrm{fst}\left(\mathrm{fst}\left(\operatorname{radec}\left(\mathrm{w}_{2}, s k_{c}\right)\right)\right)$. It holds in $\phi_{1}$ but not in $\phi_{2}$, hence, we have that $\mathcal{K}_{1} \nsubseteq_{t} \mathcal{K}_{2}$.

Consider now a variant $P_{\mathrm{NSL}}^{\prime}$ where the second message $\left\{\left\langle N_{a},\left\langle N_{b}, B\right\rangle\right\rangle\right\}_{\text {pub }(A)}$ is no longer encoded using two nested pairs but using a triple instead; that is, $\left\{\left\langle N_{a}, N_{b}, B\right\rangle^{3}\right\}_{\text {pub }(A)}$. This transformation yields the process

$$
\begin{aligned}
Q_{B}= & \operatorname{in}\left(c_{B}, \operatorname{raenc}\left(\left\langle\mathrm{a}, y_{1}\right\rangle, \operatorname{pub}\left(s k_{b}\right), y_{2}\right)\right), \\
& \operatorname{out}\left(c_{B}, \operatorname{raenc}\left(\left\langle y_{1}, n_{b}, \mathrm{~b}\right\rangle^{3}, \operatorname{pub}\left(s k_{a}\right), r_{2}\right)\right), \\
& \operatorname{in}\left(c_{B}, \operatorname{raenc}\left(n_{b}, \operatorname{pub}\left(s k_{b}\right), y_{3}\right)\right),
\end{aligned}
$$

instead of $P_{B}$. The use of triples rules out the type flaw attack, and the resulting processes are in trace equivalence. 


\section{OUR TYPING RESULTS}

Even when considering finite processes (i.e., processes without replication), the problem of checking trace equivalence is difficult due to several sources of unboundedness. One of them is the arbitrarily large size of messages that can be forged by an attacker. We propose here a simplification result that reduces the search space for attacks. Roughly, if there is an attack, then there is a well-typed attack, for a flexible notion of type that can be adapted depending on the desired result. We establish this result both for trace properties and equivalence properties (trace equivalence). Compared to the initial work of Reference [23], we extend the result from a fixed, simple signature (symmetric encryption) to a large class of cryptographic primitives that encompasses all the standard ones. Moreover, Reference [23] only applies to trace equivalence. Intuitively, proving the small attack property for trace equivalence requires to first show how to reduce the attack on a single trace. This yields a reduction result for reachability properties, such as authentication or confidentiality, which is of independent interest.

\subsection{Typing System}

We consider any type system that is consistent with substitution and unification.

Definition 3.1. A typing system is a pair $(\mathcal{T}, \delta)$ where $\mathcal{T}$ is a set of elements called types and $\delta$ is a function mapping terms $t \in \mathcal{T}_{0}\left(\Sigma_{c}, \Sigma_{0}^{+} \cup \mathcal{N} \cup \mathcal{X}\right)$ to types in $\mathcal{T}$ such that:

- If $t$ is a term of type $\tau$ and $\sigma$ is a well-typed substitution that is a substitution such that $x$ and $\sigma(x)$ have the same type, then $t \sigma$ is of type $\tau$.

- For any unifiable terms $t$ and $t^{\prime}$ with the same type, i.e., $\delta(t)=\delta\left(t^{\prime}\right)$, their most general unifier $m g u\left(t, t^{\prime}\right)$ is well-typed.

Consider a configuration $\mathcal{K}$ and a a typing system $(\mathcal{T}, \delta)$, an execution $\mathcal{K} \stackrel{\operatorname{tr}}{\Longrightarrow}(\mathcal{P} ; \phi ; \sigma ; i)$ is well-typed if $\sigma$ is a well-typed substitution.

Some interesting typing systems are structure-preserving typing systems; that is, typing systems that preserve the structure of terms. They are defined as follows:

Definition 3.2. A structure-preserving typing system is a pair $\left(\mathcal{T}_{\text {init }}, \delta\right)$ where $\mathcal{T}_{\text {init }}$ is a set of elements called initial types, and $\delta$ is a function mapping data in $\Sigma_{0}^{+} \uplus \mathcal{N} \uplus \mathcal{X}$ to types $\tau$ generated using the following grammar:

$$
\tau, \tau_{1}, \tau_{2}=\tau_{0} \mid \mathrm{f}\left(\tau_{1}, \ldots, \tau_{n}\right) \text { with } \mathrm{f} \in \Sigma_{\mathrm{c}} \text { and } \tau_{0} \in \mathcal{T}_{\text {init }} .
$$

Then, $\delta$ is extended to constructor terms as follows:

$$
\delta\left(\mathrm{f}\left(t_{1}, \ldots, t_{n}\right)\right)=\mathrm{f}\left(\delta\left(t_{1}\right), \ldots, \delta\left(t_{n}\right)\right) \text { with } \mathrm{f} \in \Sigma_{\mathrm{c}} .
$$

The following lemma proves that structure-preserving typing systems are typing systems as defined in Definition 3.1:

Lemma 3.3. Let $\left(\mathcal{T}_{\text {init }}, \delta\right)$ be a structure-preserving typing system. Then $\left(\mathcal{T}\left(\Sigma, \mathcal{T}_{\text {init }}\right), \delta\right)$ is a typing system as in Definition 3.1.

Proof. We have to prove the two following items:

(1) If $t$ is a term and $\sigma$ is a well-typed substitution, then $\delta(t \sigma)=\delta(t)$.

(2) For any unifiable terms $t$ and $t^{\prime}$ with the same type, i.e., $\delta(t)=\delta\left(t^{\prime}\right)$, their most general unifier $m g u\left(t, t^{\prime}\right)$ is well-typed.

We prove item (1) by induction on $t$. If $t$ is a name or a constant, then $t \sigma=t$ and the result trivially holds. If $t$ is a variable, then $\delta(t \sigma)=\delta(t)$, as $\sigma$ is well-typed. Now, if $t=\mathrm{f}\left(t_{1}, \ldots, t_{n}\right)$, 
then $\delta(t \sigma)=\mathrm{f}\left(\delta\left(t_{1} \sigma\right), \ldots, \delta\left(t_{n} \sigma\right)\right)$, as $\left(\mathcal{T}_{\text {init }}, \delta\right)$ is structure-preserving. By induction hypothesis, we have that $\delta\left(t_{i} \sigma\right)=\delta\left(t_{i}\right)$ for $i \in\{1, \ldots, n\}$, and we get that $\delta(t \sigma)=\mathrm{f}\left(\delta\left(t_{1}\right), \ldots, \delta\left(t_{n}\right)\right)$. As $\delta$ is structure-preserving, we have that $\delta(t)=\mathrm{f}\left(\delta\left(t_{1}\right), \ldots, \delta\left(t_{n}\right)\right)$, and this allows us to conclude.

We now prove item (2). Given a set $\Gamma$ of well-typed equations, we denote \#vars $(\Gamma)$ the number of variables occurring in $\Gamma$, and $|\Gamma|$ its size, i.e., $\sum_{t=t^{\prime} \in \Gamma}\left(|t|+\left|t^{\prime}\right|\right)$ where $|t|$ denotes the number of symbols occurring in $t$. Our measure $\|\Gamma\|$ is determined by these two elements, in lexicographic order. We prove the result by induction on $\|\Gamma\|$ relying on this measure.

Base case: $\|\Gamma\|=(0,0)$, i.e., $\Gamma=\emptyset$, and thus the result trivially holds.

Induction step: $\Gamma=\Gamma^{\prime} \uplus\left\{t=t^{\prime}\right\}$. We distinguish several cases:

- In case $t$ or $t^{\prime}$ is a variable. We assume w.l.o.g. that $t$ is a variable $x$. In such a case, let $\sigma=\left\{x \mapsto t^{\prime}\right\}$. We have that $\sigma$ is well-typed. Moreover, applying our induction hypothesis on $\Gamma^{\prime}$, we deduce that $m g u\left(\Gamma^{\prime}\right)$ is well-typed, and thus $\left\{x \mapsto t^{\prime} m g u\left(\Gamma^{\prime}\right)\right\}$ is well-typed, and $m g u(\Gamma)=m g u\left(\Gamma^{\prime}\right) \uplus\left\{x \mapsto t^{\prime} m g u\left(\Gamma^{\prime}\right)\right\}$ is well-typed.

- Otherwise, assume that $t$ is an atom (but not a variable). In such a case, we have that $t^{\prime}$ is also an atom (and not a variable due to the previous case). Therefore, since $t$ and $t^{\prime}$ are unifiable, we have that $t=t^{\prime}$, and $m g u(\Gamma)=m g u\left(\Gamma^{\prime}\right)$. Since $\left\|\Gamma^{\prime}\right\|<\|\Gamma\|$, we have that $m g u\left(\Gamma^{\prime}\right)$ is well-typed by induction hypothesis, and this allows us to conclude.

- Now, we assume that $t=\mathrm{f}\left(t_{1}, \ldots, t_{k}\right)$. In such a case, we have that $t^{\prime}=\mathrm{f}\left(t_{1}^{\prime}, \ldots, t_{k}^{\prime}\right)$, since $t^{\prime}$ is not a variable and we know that $t$ and $t^{\prime}$ are unifiable. We have that $m g u(\Gamma)=m g u\left(\Gamma^{\prime \prime}\right)$ where $\Gamma^{\prime \prime}=\Gamma^{\prime} \uplus\left\{t_{1}=t_{1}^{\prime}, \ldots, t_{k}=t_{k}^{\prime}\right\}$. Note that $\Gamma^{\prime \prime}$ is a set of well-typed equations, $\left\|\Gamma^{\prime \prime}\right\|<$ $\|\Gamma\|$ and thus $m g u\left(\Gamma^{\prime \prime}\right)$ is well-typed by induction hypothesis.

This concludes the proof.

We further assume the existence of an infinite number of constants in $\Sigma_{0}$ (resp. $\Sigma_{\text {fresh }}^{\text {atom }}, \Sigma_{\text {fresh }}^{\text {bitstring }}$ ) of any type.

Example 3.4. Let us continue our running example, with the processes $\bar{P}_{\mathrm{NSL}}^{1}$ and $\bar{P}_{\mathrm{NSL}}^{2}$, as defined in Example 2.12. We consider the structure-preserving typing system generated from the set $\mathcal{T}_{\mathrm{NSL}}=$ $\left\{\tau_{\mathrm{a}}, \tau_{\mathrm{b}}, \tau_{\mathrm{c}}, \tau_{n a}, \tau_{n b}, \tau_{r}, \tau_{s k}, \tau_{k}\right\}$ of initial types, and the function $\delta_{\mathrm{NSL}}$ that associates the expected type to each constant/name $\left(\delta_{\mathrm{NSL}}(a)=\tau_{a}, \delta_{\mathrm{NSL}}(b)=\tau_{b}\right.$, etc.), and the following type to the variables:

$$
\delta_{\mathrm{NSL}}\left(y_{1}\right)=\delta_{\mathrm{NSL}}\left(y_{1}^{\prime}\right)=\tau_{n a}, \text { and } \delta_{\mathrm{NSL}}\left(y_{2}\right)=\delta_{\mathrm{NSL}}\left(y_{3}\right)=\delta_{\mathrm{NSL}}\left(y_{2}^{\prime}\right)=\delta_{\mathrm{NSL}}\left(y_{3}^{\prime}\right)=\tau_{r} .
$$

The goal of our main results is to be able to consider only execution traces that comply with a given type, like this one.

\subsection{Type Compliance}

Our main assumption on the typing of protocols is that any two unifiable encrypted subterms are of the same type. The goal of this part is to state this hypothesis formally. For this, we need to define the notion of encrypted subterms.

Among the constructor symbols in $\Sigma_{\mathrm{c}}$, we distinguish those that are transparent. They intuitively correspond to constructors that can be freely opened by the attacker, such as pairs, tuples, or lists. A constructor symbol $\mathrm{f}$ of arity $n$ is transparent if there exists a term $\mathrm{f}\left(R_{1}^{\mathrm{f}}, \ldots, R_{n}^{\mathrm{f}}\right) \in \mathcal{T}(\Sigma, \square)$ such that for any term $t \in \mathcal{T}_{0}\left(\Sigma, \Sigma_{0}^{+} \uplus \mathcal{N} \uplus \mathcal{X}\right)$ such that $\operatorname{root}(t)=\mathrm{f}$, we have that $\mathrm{f}\left(R_{1}^{\mathrm{f}}, \ldots, R_{n}^{\mathrm{f}}\right)\{\square \rightarrow$ $t\} \downarrow=t$. We denote $C_{\mathrm{f}}$ such a term, and write $C_{\mathrm{f}}[t]$ the term obtained by replacing each occurrence of the hole with $t$. 
We write $E S t(t)$ for the set of encrypted subterms of $t$, i.e., the set of subterms that are not headed by a transparent function.

$$
E S t(t)=\left\{u \in S t(t) \mid u \text { is of the form } \mathrm{f}\left(u_{1}, \ldots, u_{n}\right) \text { and } \mathrm{f} \text { is not transparent }\right\}
$$

Example 3.5. Going back to the signature $\Sigma^{\mathrm{ex}}$ introduced in Example 2.1, the symbols \langle\rangle and \langle\rangle$^{3}$ are transparent: the contexts $C_{\langle\rangle}=\langle$fst $(\square)$, snd $(\square)\rangle$ and $C_{\langle\rangle^{3}}=\left\langle\operatorname{proj}_{1}^{3}(\square), \operatorname{proj}_{2}^{3}(\square), \operatorname{proj}_{3}^{3}(\square)\right\rangle^{3}$ satisfy the requirements.

A configuration $\mathcal{K}=(\mathcal{P} ; \phi ; \emptyset ; i)$ is type-compliant if two unifiable encrypted subterms occurring in $\mathcal{K}$ (i.e., either in $\mathcal{P}$ or in $\phi$ ) have the same type. Formally, we use the definition given in the preliminary result [23], which is similar to the one originally introduced by B. Blanchet and A. Podelski in Reference [15].

Definition 3.6. A configuration $\mathcal{K}$ is type-compliant w.r.t. a typing system $\left(\mathcal{T}_{\text {init }}, \delta\right)$ if for every $t, t^{\prime} \in \operatorname{ESt}(\mathcal{K})$, we have that: $t$ and $t^{\prime}$ unifiable implies that $\delta(t)=\delta\left(t^{\prime}\right)$.

Example 3.7. Continuing our running example with $\mathcal{K}_{\mathrm{NSL}}=\left(P_{\mathrm{NSL}} ; \phi_{0} ; \emptyset ; 0\right)$, we have that $\mathcal{K}_{\mathrm{NSL}}$ is not type-compliant w.r.t. the typing system given in Example 3.4. Indeed, the encrypted subterms are:

- $t_{1}=\operatorname{raenc}\left(\left\langle\mathrm{a}, y_{1}\right\rangle, \operatorname{pub}\left(s k_{b}\right), y_{2}\right)$ and $t_{1}^{\prime}=\operatorname{raenc}\left(\left\langle\mathrm{c}, y_{1}^{\prime}\right\rangle, \operatorname{pub}\left(s k_{a}\right), y_{2}^{\prime}\right)$;

- $t_{2}=\operatorname{raenc}\left(\left\langle y_{1},\left\langle n_{b}, \mathrm{~b}\right\rangle\right\rangle, \operatorname{pub}\left(s k_{a}\right), r_{2}\right)$ and $t_{2}^{\prime}=\operatorname{raenc}\left(\left\langle y_{1}^{\prime},\left\langle n_{b}^{\prime}, \mathrm{a}\right\rangle\right\rangle, \operatorname{pub}\left(s k_{c}\right), r_{2}^{\prime}\right)$;

- $t_{3}=\operatorname{raenc}\left(n_{b}, \operatorname{pub}\left(s k_{b}\right), y_{3}\right)$ and $t_{3}^{\prime}=\operatorname{raenc}\left(n_{b}^{\prime}, \operatorname{pub}\left(s k_{a}\right), y_{3}^{\prime}\right)$;

- $t_{A}=\operatorname{pub}\left(s k_{a}\right)$ and $t_{B}=\operatorname{pub}\left(s k_{b}\right)$.

Actually, we have that $t_{2}$ and $t_{1}^{\prime}$ are unifiable with $\sigma=\left\{y_{1} \rightarrow \mathrm{c}, y_{1}^{\prime} \rightarrow\left\langle n_{b}, \mathrm{~b}\right\rangle, y_{2}^{\prime} \rightarrow r_{2}\right\}$, but we have that:

- $\delta_{\mathrm{NSL}}\left(t_{2}\right)=\operatorname{raenc}\left(\left\langle\tau_{n a},\left\langle\tau_{n b}, \tau_{\mathrm{b}}\right\rangle\right\rangle, \operatorname{pub}\left(\tau_{s k}\right), \tau_{r}\right)$, whereas

- $\delta_{\mathrm{NSL}}\left(t_{1}^{\prime}\right)=\operatorname{raenc}\left(\left\langle\tau_{\mathrm{c}}, \tau_{n a}\right\rangle, \operatorname{pub}\left(\tau_{s k}\right), \tau_{r}\right)$.

Actually, $\mathcal{K}_{\mathrm{NSL}}$ is type-compliant when considering a typing system such that $\delta_{\mathrm{NSL}}\left(y_{1}^{\prime}\right)=$ $\left\langle\tau_{n b}, \tau_{b}\right\rangle, \delta_{\mathrm{NSL}}\left(y_{1}\right)=\tau_{c}$, and keeping the other elements as defined in the typing system introduced in Example 3.4. Note that, w.r.t. this typing system, the attack trace $\operatorname{tr}$ given in Example 2.7 is well-typed.

Consider now the variant $P_{\mathrm{NSL}}^{\prime}$, as sketched in Example 2.12, where a triple is used instead of two pairs; that is, replacing messages $t_{2}$ and $t_{2}^{\prime}$ by $s_{2}=\operatorname{raenc}\left(\left\langle y_{1}, n_{b}, \mathrm{~b}\right\rangle^{3}, \operatorname{pub}\left(s k_{a}\right), r_{2}\right)$ and $s_{2}^{\prime}=$ raenc $\left(\left\langle y_{1}^{\prime}, n_{b}^{\prime}, \mathrm{a}\right\rangle^{3}, \operatorname{pub}\left(s k_{c}\right), r_{2}^{\prime}\right)$. Then the corresponding configuration $\mathcal{K}_{\mathrm{NSL}}^{\prime}=\left(P_{\mathrm{NSL}}^{\prime} ; \phi_{0} ; \emptyset ; 0\right)$ is type-compliant w.r.t. the typing system given in Example 3.4.

\subsection{Reduction Results}

Our main result consists in showing that whenever there is an attack, then there is an attack that is well-typed. This holds for reachability properties as well as equivalence properties.

3.3.1 Reachability. We first prove that for any execution trace, there is a well-typed execution that follows the same sequence of input and output, on the same channels. Formally, we define $\overline{\operatorname{tr}}$ obtained from $\operatorname{tr}$ by replacing any action in $(c, R)$ by in $\left(c,{ }_{-}\right)$, any out $(c, \mathrm{w})$ by out $\left(c,{ }_{-}\right)$, while phase $i^{\prime}$ is left unchanged. Intuitively, $\overline{\operatorname{tr}}$ only remembers the type of actions, and on which channel.

TheOREM 3.8. Let $\mathcal{K}_{P}$ be a $\Sigma_{0}^{-}$-configuration type-compliant w.r.t. $\left(\mathcal{T}_{0}, \delta_{0}\right)$. If $\mathcal{K}_{P} \stackrel{\operatorname{tr}}{\Longrightarrow}(\mathcal{P} ; \phi ; \sigma ; i)$ w.r.t. $\Sigma_{0}^{-}$, then there exists a well-typed execution $\mathcal{K}_{P} \stackrel{\operatorname{tr}^{\prime}}{\Longrightarrow}\left(\mathcal{P} ; \phi^{\prime} ; \sigma^{\prime} ; i\right)$ w.r.t. $\Sigma_{0}^{+}$such that $\overline{\operatorname{tr}^{\prime}}=\overline{\operatorname{tr}}$. 
Conversely, if $\mathcal{K}_{P} \stackrel{\operatorname{tr}^{\prime}}{\Longrightarrow}\left(\mathcal{P} ; \phi^{\prime} ; \sigma^{\prime} ; i\right)$ is a well-typed execution w.r.t. $\Sigma_{0}^{+}$, then there exists $\mathcal{K}_{P} \stackrel{\operatorname{tr}}{\Longrightarrow}$ $(\mathcal{P} ; \phi ; \sigma ; i)$ w.r.t. $\Sigma_{0}^{-}$such that $\overline{\mathrm{tr}}=\overline{\mathrm{tr}^{\prime}}$.

This shows that for any property that can be expressed as a reachability property, it is sufficient to consider well-typed attacks. For example, secrecy of a data $s$ can easily be encoded by adding a witness process of the form $\operatorname{in}(c, s)$.out $\left(c_{\text {secret-violated }}, s\right)$. Then the secret of $s$ is preserved if and

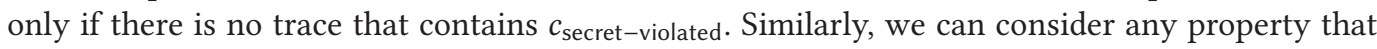
expresses that some state should never be reached. The second part of Theorem 3.8 can be easily established by replacing constants from $\Sigma_{\text {fresh }}$ (used in the witness) by "fresh" constants from $\Sigma_{0}^{-}$ preserving their sort. The resulting trace is still a valid execution trace. The first and main part of this theorem is proved in Section 4.

3.3.2 Equivalence. Similarly to reachability, we show that whenever two processes are not in trace equivalence, then there is a well-typed witness of non-equivalence. Actually, we prove this result for the notion of trace inclusion given in Definition 2.10. Formally, assume given two $\Sigma_{0}^{-}-$ configurations $\mathcal{K}_{P}$ and $\mathcal{K}_{Q}$ such that $\mathcal{K}_{P} \nsubseteq_{t} \mathcal{K}_{Q}$ w.r.t. $\Sigma_{0}^{-}$. A witness of non-inclusion is a trace $(\operatorname{tr}, \phi) \in \operatorname{trace}_{\Sigma_{0}^{-}}\left(\mathcal{K}_{P}\right)$ such that:

- either there is no $\psi$ such that $(\operatorname{tr}, \psi) \in \operatorname{trace}_{\Sigma_{0}^{-}}\left(\mathcal{K}_{Q}\right)$;

- or $\phi \nsubseteq_{s} \psi$ w.r.t. $\Sigma_{0}^{-}$for any $\psi$ such that $(\operatorname{tr}, \psi) \in \operatorname{trace}_{\Sigma_{0}^{-}}\left(\mathcal{K}_{Q}\right)$.

Note that when a configuration is determinate, once the sequence tr is fixed, there is a unique frame reachable through tr up to static equivalence.

Theorem 3.9. Let $\mathcal{K}_{P}$ be a $\Sigma_{0}^{-}$-configuration type-compliant w.r.t. $\left(\mathcal{T}_{0}, \delta_{0}\right)$ and $\mathcal{K}_{Q}$ be another $\Sigma_{0}^{-}$-configuration. We have that $\mathcal{K}_{P} \ddagger_{t} \mathcal{K}_{Q}$ w.r.t. $\Sigma_{0}^{-}$with witness $(\operatorname{tr}, \phi)$ if, and only if, there exists a witness $\left(\operatorname{tr}^{\prime}, \phi^{\prime}\right) \in$ trace $_{\Sigma_{0}^{+}}\left(\mathcal{K}_{P}\right)$ of this non-inclusion such that its underlying execution $\mathcal{K}_{P} \stackrel{\operatorname{tr}^{\prime}}{\Longrightarrow}$ $\left(\mathcal{P} ; \phi^{\prime} ; \sigma ; i\right)$ w.r.t. $\Sigma_{0}^{+}$is well-typed and $\overline{\mathrm{tr}}=\overline{\mathrm{tr}^{\prime}}$.

The main part of the theorem is proved in Section 5. Regarding the fact that the existence of a witness w.r.t. $\Sigma_{0}^{+}$can be turned into a witness w.r.t. $\Sigma_{0}^{-}$, the proof can be done as in the reachability case. This result can be easily established by replacing any symbol from $\Sigma_{\text {fresh }}$ by a symbol of $\Sigma_{0}^{-}$ preserving sorts.

\section{TYPING RESULT FOR REACHABILITY}

The goal of this section is to provide the main ingredients of the proof of Theorem 3.8. Some of the lemmas are also useful for the proof of Theorem 3.9 on equivalence.

We first define a notion of simple recipes and we explain how we can restrict ourselves to simple recipes (see Section 4.1). Second, our proof crucially relies on a crafted measure on recipes that we introduce in Section 4.2. A witness of reachability, minimal w.r.t. this measure, will be shown to satisfy some good properties, used to derive a well-typed trace.

\subsection{Some Preliminaries}

We introduce the notion of forced normal form, denoted $u \downarrow$. This is the normal form obtained when applying rewrite rules as soon as the destructor and the constructor match, for example decrypting a message even with a wrong key. Formally, we define the forced rewriting system associated to a set $\mathcal{R}$ of rewriting rules. 
Definition 4.1. Given a rewriting rule of the form $\ell_{\text {des }} \rightarrow r_{\text {des }}$ as defined in Section 2.1, its associated forced rewriting rule is $\ell_{\text {des }}^{\prime} \rightarrow r_{\text {des }}$ where $\ell_{\text {des }}^{\prime}$ is obtained from $\ell_{\text {des }}$ by keeping only the path to $r_{\text {des }}$ in $\ell_{\text {des }}$. Formally, $\ell_{\text {des }}^{\prime}$ is defined as follows:

(1) $\ell_{\text {des }}^{\prime}=\operatorname{des}\left(x_{1}, \ldots, x_{n}\right)$ when $r_{\text {des }}$ is a ground term;

(2) otherwise denoting $p_{0}$ the unique position of $\ell_{\text {des }}$ such that $\left.\ell_{\text {des }}\right|_{p_{0}}=r_{\text {des }}$ and letting $p_{0}=$ 1. $p_{0}^{\prime}$, we have that $\ell_{\text {des }}^{\prime}$ is the linear term such that:

- for any position $p^{\prime}$ prefix of $p_{0}$, we have that $\operatorname{root}\left(\ell_{\text {des }}^{\prime} \mid p^{\prime}\right)=\operatorname{root}\left(\left.\ell_{\operatorname{des}}\right|_{p^{\prime}}\right)$;

- $\left.\ell_{\text {des }}^{\prime}\right|_{p_{0}}=r_{\text {des }}$;

- for any other position $p^{\prime}$ of $\ell_{\text {des }}^{\prime}$, we have that $\left.\ell_{\text {des }}^{\prime}\right|_{p^{\prime}}$ is a variable.

We may note that the forced rewriting system associated to a rewriting system as defined in Section 2.1 is well-defined. In particular, given a rewriting rule $\operatorname{des}\left(t_{1}, \ldots, t_{n}\right) \rightarrow r_{\text {des }}$ such that $r_{\text {des }}$ is a non-ground term, there exists a unique position $p_{0}^{\prime}$ in $t_{1}$ such that $\left.t_{1}\right|_{p_{0}^{\prime}}=r_{\text {des }}$. This comes from the fact that $r_{\text {des }} \in S t\left(t_{1}\right)$, and the variable occurring in $r_{\text {des }}$ has a unique occurrence in $t_{1}$, which is a linear term.

Example 4.2. Going back to our running example, we have that the forced rewriting system $\mathcal{R}_{f}^{\mathrm{ex}}$ associated to $\mathcal{R}^{\mathrm{ex}}$ is:

$$
\operatorname{radec}\left(\operatorname{raenc}\left(x, y_{1}, z\right), y_{2}\right) \rightarrow x \quad \operatorname{fst}(\langle x, y\rangle) \rightarrow x \quad \operatorname{snd}(\langle x, y\rangle \rightarrow y .
$$

Regarding symmetric encryption and signature as introduced in Example 2.4, we get:

$$
\operatorname{sdec}\left(\operatorname{senc}\left(x, y_{1}\right), y_{2}\right) \rightarrow x \quad \operatorname{getmsg}(\operatorname{sign}(x, y)) \rightarrow x \quad \operatorname{check}\left(x_{1}, x_{2}\right) \rightarrow \text { ok. }
$$

Then, given a set $\mathcal{R}_{f}$ of rewriting rules, a term $u$ can be rewritten to $v$ using $\mathcal{R}_{f}$ if there is a position $p$ in $u$, and a rewriting rule $g\left(t_{1}, \ldots, t_{n}\right) \rightarrow t$ in $\mathcal{R}_{f}$ such that $\left.u\right|_{p}=\mathrm{g}\left(t_{1}, \ldots, t_{n}\right) \theta$ for some substitution $\theta$, and $v=u[t \theta]_{p}$. As usual, we denote $\rightarrow^{*}$, the reflexive-transitive closure of $\rightarrow$. We may note that such a rewriting system is confluent as it terminates and has no critical pair. As usual, the normal form of a term $u$ is denoted $u \downarrow$.

The forced rewriting system allows more rewriting steps than the original one. We will apply it on recipes to simplify them and avoid detours. The following lemma ensures that the term deduced (in a given frame $\phi$ ) through the recipe $R$ would be the same as the one deduced relying on $R \downarrow$ as soon as we know that $R \phi \downarrow$ is a message.

LEMmA 4.3. Let $\phi$ be a $\Sigma_{0}$-frame, $R$ a $\Sigma_{0}$-recipe such that $R \phi \downarrow$ is a $\Sigma_{0}$-message, and $R^{\prime}$ be such that $R \rightarrow R^{\prime}$. We have that $R^{\prime}$ is a $\Sigma_{0}$-recipe, and $R^{\prime} \phi \downarrow=R \phi \downarrow$.

In our development, we will consider recipes that have a simple form: they are built using constructor symbols on top of recipes that necessarily extract a subterm of the frame (roughly a recipe made of destructors).

Definition 4.4. A $\Sigma_{0}$-recipe $R$ is a subterm $\Sigma_{0}$-recipe if for any $\Sigma_{0}$-frame $\phi$ such that $R \phi \downarrow$ is a $\Sigma_{0}$-message, we have that $R \phi \downarrow \in S t(\phi)$. We say that $R$ is a simple $\Sigma_{0}$-recipe if $R=C\left[R_{1}, \ldots, R_{k}\right]$ for some context $C$ built using symbols from $\Sigma_{\mathrm{c}} \uplus \Sigma_{0}$, and each $R_{i}$ is a subterm $\Sigma_{0}$-recipe such that $\operatorname{root}\left(R_{i}\right) \notin \Sigma_{\mathrm{c}}$.

Simple recipes can be obtained through normalization w.r.t. our forced rewriting system.

LEMMA 4.5. Let $\theta$ be a substitution with $\operatorname{dom}(\theta) \subseteq \Sigma_{\text {fresh }}$ and whose image contains $\Sigma_{0}^{-}$-recipes. Let $R$ be a $\Sigma_{0}^{+}$-recipe in normal form w.r.t. $\rightarrow$ such that $(R \theta) \phi \downarrow$ is a $\Sigma_{0}^{+}$-message for some $\Sigma_{0}^{-}$-frame $\phi$. We have that $R^{\prime}$ is a simple $\Sigma_{0}^{+}$-recipe for any $R^{\prime} \in S t(R)$. 


\subsection{Our Measure}

One key step of the proof is to design a measure that reflects how to transform a trace into a welltyped one. Our transformation will proceed by modifying the recipes used by the attacker to forge messages: Instead of sending arbitrarily large messages, he should send only small, well-typed messages. The transformation depends on the trace and the frame $\phi_{S}$ under consideration, and therefore our measure is parameterized by $\phi_{S}$. Finally, the measure of a recipe will be determined by three elements, in lexicographic order.

- First, the size of the term computed by $R$, that is $R \phi_{S} \downarrow$;

- Second, the recipe $R$ should be headed by as much constructor terms as possible (In particular, we will prefer the recipe $\langle$ fst $(w)$, snd $(w)\rangle$ over w itself);

- Finally, the size of $R$ itself.

The rest of this section is devoted to the definition of our measure and the establishment of a couple of its properties.

Given a term $t \in \mathcal{T}\left(\Sigma, \Sigma_{0}^{+} \uplus \mathcal{N}\right)$, we denote $\operatorname{Multi}(t)$, the multiset of elements from $\Sigma \uplus \Sigma_{0}^{+} \uplus \mathcal{N}$ defined as follows:

- $\operatorname{Multi}(a)=\{a\}$ when $a \in \Sigma_{0}^{+} \uplus \mathcal{N}$, and

- Multi $\left(f\left(t_{1}, \ldots, t_{n}\right)\right)=\{\mathrm{f}\} \uplus \operatorname{Multi}\left(t_{1}\right) \uplus \ldots \operatorname{Multi}\left(t_{n}\right)$ when $\mathrm{f} \in \Sigma$.

Given a set $D$ of data and a term $t \in \mathcal{T}(\Sigma, D)$, the size of $t$, denoted $|t|$, is the number of function symbols occurring in it. The hat of $t$ is the constructor context $R$ (i.e., a term built on $\Sigma_{\mathrm{c}}$ with some holes) such that $t=R\left[t_{1}, \ldots, t_{n}\right]$ with $\operatorname{root}\left(t_{1}\right), \ldots, \operatorname{root}\left(t_{n}\right) \notin \Sigma_{\mathrm{c}}$. We denote it hat $(t)$.

Given a $\Sigma_{0}^{+}$-frame $\phi_{S}$ together with an ordering $\prec$ on $\Sigma_{\text {fresh }}$ (typically the one corresponding to the order of appearance of these constants in the underlying trace $\operatorname{tr}_{S}$ associated to $\phi_{S}$ ), the measure $\mu \phi_{S}$ associated to a $\Sigma_{0}^{+}$- recipe $R$ is defined as follows (using the lexicographic ordering):

(1) $\mu_{\phi_{S}}^{1}(R)=\operatorname{Multi}\left(R \phi_{S} \downarrow\right)$ where elements of the multisets are ordered as follows $\left(c_{\min } \in \Sigma_{0}^{-}\right)$:

$$
c_{\min }<\Sigma_{0}^{-} \backslash\left\{c_{\min }\right\} \uplus \Sigma_{\mathrm{c}}<\Sigma_{\text {fresh }}<\Sigma_{\mathrm{d}},
$$

and for elements in $\sum_{\text {fresh }}$, we have that $c<c^{\prime}$ when $c<c^{\prime}$.

(2) $\mu_{\phi_{S}}^{2}(R)=\left|R \phi_{S} \downarrow\right|-\mid$ hat $(R) \mid$.

(3) $\mu^{3}(R)=|R|$.

Note that $c_{\min } \in \Sigma_{0}^{-}$is the minimal $\Sigma_{0}^{-}$-recipe according to this measure.

We start by establishing some properties regarding this measure. First, we may note that the measure $\mu_{\phi_{S}}^{2}$ remains positive, since, intuitively, the hat of a term can never disappear by rewriting. (This result is formally stated and proved in Appendix A.2-see Lemma A.1).

Then we can show that if a recipe $R_{2}$ is greater than a recipe $R_{1}$ and does not yield a message, then $R_{0}\left[R_{2}\right]$ is greater than $R_{0}\left[R_{1}\right]$. This technical lemma will be used very often in our proofs, when reasoning about some reduction that failed inside a bigger term.

LEMmA 4.6. Let $<$ be an ordering on $\Sigma_{\mathrm{fresh}}$, $\phi_{S}$ be a $\Sigma_{0}^{+}$-frame, $R_{1}, R_{2}$ be two $\Sigma_{0}^{+}$-recipes such that $R_{2} \phi_{S} \downarrow$ is not a $\Sigma_{0}^{+}$-message, and $\mu_{\phi_{S}}^{1}\left(R_{1}\right)<\mu_{\phi_{S}}^{1}\left(R_{2}\right)$. Let $R_{0}$ be a $\Sigma_{0}^{+}$-recipe, and p a position in $R_{0}$. We have that:

$$
\mu_{\phi_{S}}^{1}\left(R_{0}\left[R_{1}\right]_{p}\right)<\mu_{\phi_{S}}^{1}\left(R_{0}\left[R_{2}\right]_{p}\right) .
$$

The proof of Lemma 4.6 is given in Appendix A.2. It relies on the fact that $R_{0}\left[R_{1}\right]$ may only reduce more than $R_{0}\left[R_{2}\right]$.

The measure decreases when applying forced reduction. 
Lemma 4.7. Let $<$ be an ordering on $\Sigma_{\text {fresh }}$, $\phi_{S}$ be a $\Sigma_{0}^{+}$-frame, and $R, R^{\prime}$ be two $\Sigma_{0}^{+}$-recipes such that $R \rightarrow R^{\prime}$. We have that $\mu_{\phi_{S}}\left(R^{\prime}\right)<\mu_{\phi_{S}}(R)$.

The proof of Lemma 4.7 is given in Appendix A.2. Intuitively, if $R \rightarrow R^{\prime}$, then we consider the instantiated rule $\ell_{\text {des }} \theta \rightarrow r_{\text {des }} \theta$ that has been applied. Either $\ell_{\text {des }} \theta \phi_{S} \downarrow$ is a message. Then both $R$ and $R^{\prime}$ yield the same term, which is $R \phi_{S} \downarrow=R^{\prime} \phi_{S} \downarrow$. The first two items of the measure are unchanged, and we conclude thanks to the third item, since $R^{\prime}$ is smaller than $R$. In case $\ell_{\text {des }} \theta \phi_{S} \downarrow$ is not a message, we conclude by Lemma 4.6.

\subsection{Completeness}

We are now ready to prove the core part of Theorem 3.8.

THEOREM 3.8. Let $\mathcal{K}_{P}$ be a $\Sigma_{0}^{-}$-configuration type-compliant w.r.t. $\left(\mathcal{T}_{0}, \delta_{0}\right)$. If $\mathcal{K}_{P} \stackrel{\operatorname{tr}}{\Longrightarrow}(\mathcal{P} ; \phi ; \sigma ; i)$ w.r.t. $\Sigma_{0}^{-}$, then there exists a well-typed execution $\mathcal{K}_{P} \stackrel{\operatorname{tr}^{\prime}}{\Longrightarrow}\left(\mathcal{P} ; \phi^{\prime} ; \sigma^{\prime} ; i\right)$ w.r.t. $\Sigma_{0}^{+}$such that $\overline{\operatorname{tr}^{\prime}}=\overline{\operatorname{tr}}$.

Conversely, if $\mathcal{K}_{P} \stackrel{\operatorname{tr}^{\prime}}{\Longrightarrow}\left(\mathcal{P} ; \phi^{\prime} ; \sigma^{\prime} ; i\right)$ is a well-typed execution w.r.t. $\Sigma_{0}^{+}$, then there exists $\mathcal{K}_{P} \stackrel{\operatorname{tr}}{\Longrightarrow}$ $(\mathcal{P} ; \phi ; \sigma ; i)$ w.r.t. $\Sigma_{0}^{-}$such that $\overline{\mathrm{tr}}=\overline{\mathrm{tr}^{\prime}}$.

Given a trace $\mathcal{K}_{P} \stackrel{\operatorname{tr}}{\Longrightarrow}(\mathcal{P} ; \phi ; \sigma ; i)$, we need to build a well-typed trace $\mathcal{K}_{P} \stackrel{\operatorname{tr}}{\Longrightarrow}\left(\mathcal{P} ; \phi_{S} ; \sigma_{S} ; i\right)$ such that $\overline{\operatorname{tr}_{S}}=\overline{\operatorname{tr}}$. This is done inductively in the number of execution steps, by modifying the recipes used by the adversary: Given a recipe $R$, some parts of $R$ will be replaced by fresh constants from $\Sigma_{\text {fresh. }}$. Roughly, we will show that $\operatorname{tr}$ is actually an instance of $\operatorname{tr}_{S}$ in which the fresh constants of $\operatorname{tr}_{S}$ are replaced by recipes over the current frame $\phi_{S}$.

We first note that once the recipes are fixed (by $\theta$ in the following lemma), the underlying terms-computed by the recipes-are entirely determined.

Lemma 4.8. Let $\phi_{S}$ be a $\Sigma_{0}^{+}$-frame together with $<$a total ordering on $\operatorname{dom}\left(\phi_{S}\right)$. Let $\theta$ be a substitution such that $\operatorname{dom}(\theta) \subseteq \Sigma_{\text {fresh }}$, and for any $c \in \Sigma_{\text {fresh }}$ occurring in $\phi_{S}$, we have that $c \in \operatorname{dom}(\theta)$ and $c \theta \in \mathcal{T}\left(\Sigma, \Sigma_{0}^{-} \uplus \operatorname{dom}\left(\phi_{S}\right)\right)$. Moreover, we assume that in case $c \in \Sigma_{\text {fresh }}$ occurs in $\mathrm{w} \phi_{S}$, then $\mathrm{w}^{\prime} \prec \mathrm{w}$ for any $\mathrm{w}^{\prime} \in \operatorname{vars}(c \theta)$. We consider the substitution $\lambda$ whose domain is $\operatorname{dom}(\theta)$, and such that:

$$
c \lambda=(c \theta)\left(\phi_{S} \lambda\right) \downarrow \text { for any } c \in \operatorname{dom}(\lambda) .
$$

The substitution $\lambda$ is well-defined. Moreover, if $(c \theta) \phi_{S} \downarrow$ is a $\Sigma_{0}^{+}$-message for each $c \in \operatorname{dom}(\theta)$, and $(c \theta) \phi_{S} \downarrow$ is an atomic $\Sigma_{0}^{+}$-message when $c \in \Sigma_{\text {fresh }}^{\text {atom }}$, then $c \lambda$ is a $\Sigma_{0}^{-}$-message for each $c \in \operatorname{dom}(\lambda)$, and $c \lambda$ is an atomic $\Sigma_{0}^{-}$-message when $c \in \sum_{\text {fresh }}^{\text {atom }}$.

We call $\lambda$ the first-order substitution associated to $\theta$ through $\phi_{S}$.

The fact that $\lambda$ is well-defined comes from the recursive application of the recipes defined by $\theta$, thanks to the order on the variables. This lemma is formally proved in Appendix A.3.

The relation $c \lambda=(c \theta)\left(\phi_{S} \lambda\right) \downarrow$ established by Lemma 4.8 can be generalized to arbitrary recipes $R$.

Lemma 4.9. Let $\phi_{S}$ be a $\Sigma_{0}^{+}$-frame together with $<$a total ordering on dom $\left(\phi_{S}\right)$. Let $\theta$ be a substitution such that $\operatorname{dom}(\theta) \subseteq \Sigma_{\text {fresh }}$, and for any $c \in \sum_{\text {fresh }}$ occurring in $\phi_{S}$ we have that $c \in \operatorname{dom}(\theta)$ and $c \theta \in \mathcal{T}\left(\Sigma, \Sigma_{0}^{-} \uplus \operatorname{dom}\left(\phi_{S}\right)\right)$. Moreover, we assume that in case $c \in \sum_{\text {fresh }}$ occurs in $\mathrm{w} \phi_{S}$, then $\mathrm{w}^{\prime} \prec \mathrm{w}$ for any $\mathrm{w}^{\prime} \in \operatorname{vars}(c \theta)$. Let $\lambda$ be the first-order substitution associated to $\theta$ through $\phi_{S}$.

Assume that for any $c \in \operatorname{dom}(\lambda)$, we have that $c \lambda$ is a $\Sigma_{0}^{-}$-message. Moreover, $c \lambda$ is an atomic $\Sigma_{0}^{-}$ message when $c \in \Sigma_{\text {fresh }}^{\text {atom }}$. Let $R \in \mathcal{T}\left(\Sigma, \Sigma_{0}^{-} \uplus \operatorname{dom}(\theta) \uplus \operatorname{dom}\left(\phi_{S}\right)\right)$ such that $R \phi_{S} \downarrow$ is a $\Sigma_{0}^{+}$-message. We have that $(R \theta)\left(\phi_{S} \lambda\right) \downarrow=\left(R \phi_{S} \downarrow\right) \lambda$.

The proof follows from an induction on $R$. The base case is ensured by Lemma 4.8. A formal proof is given in Appendix A.3. 
We also note that execution traces do not introduce new encrypted subterms: They are all instances of the initial encrypted subterms.

Lemma 4.10. Let $\mathcal{K}_{0}=\left(\mathcal{P}_{0} ; \phi_{0} ; \emptyset ; 0\right)$ be an initial $\Sigma_{0}$-configuration and $\mathcal{K}=(\mathcal{P} ; \phi ; \sigma ; i)$ be a $\Sigma_{0}$ configuration such that $\mathcal{K}_{0} \stackrel{\operatorname{tr}}{\Longrightarrow} \mathcal{K}$ for some $\operatorname{tr}$ w.r.t. $\Sigma_{0}$.

(1) We have that $\operatorname{ESt}(\mathcal{K} \sigma) \subseteq \operatorname{ESt}\left(\mathcal{K}_{0} \sigma\right)$.

(2) Moreover, in case $\sigma$ is an mgu between pairs of terms occurring in $\operatorname{ESt}\left(\mathcal{K}_{0}\right)$, then we have that $\operatorname{ESt}(\mathcal{K} \sigma) \subseteq \operatorname{ESt}\left(\mathcal{K}_{0}\right) \sigma$.

The first property follows from the definition: The processes of $\mathcal{K}$ are included in those of $\mathcal{K}_{0}$, and the output terms stored in the frame $\phi$ of $\mathcal{K}$ appear initially in the processes of $\mathcal{K}_{0}$. The second property comes from the fact that unification does not create new a encrypted subterm. A formal proof is given in Appendix A.3.

Given $\mathcal{K}_{0}=\left(\mathcal{P}_{0} ; \phi_{0} ; \emptyset ; \emptyset\right)$ is an initial $\Sigma_{0}^{-}$-configuration and $\left(\operatorname{tr}_{S}, \phi_{S}\right) \in$ trace $_{\Sigma_{0}^{+}}\left(\mathcal{K}_{0}\right)$, we consider the order $\prec$ induced by $\operatorname{tr}_{S}$. It is defined on the subset of $\mathcal{W} \uplus \Sigma_{\text {fresh }}$ that correspond to elements that occur in $\operatorname{tr}_{S}$ and $\phi_{S}$ as follows:

$$
u \prec v \Leftrightarrow\left\{\begin{array}{l}
\text { either } u \in \operatorname{dom}\left(\phi_{0}\right) \text { and } v \notin \operatorname{dom}\left(\phi_{0}\right), \\
\text { or } u \text { occurs in } \operatorname{tr}_{S} \text { before the first occurrence of } v \text { in } \operatorname{tr}_{S} .
\end{array}\right.
$$

We are now ready to state how we transform a trace $(\operatorname{tr}, \phi)$ into a well-typed trace $\left(\operatorname{tr}_{S}, \phi_{S}\right)$.

Proposition 4.11. Let $\mathcal{K}_{P}=\left(\mathcal{P}_{0} ; \phi_{0} ; \emptyset ; i_{0}\right)$ be an initial $\Sigma_{0}^{-}$-configuration, and $(\operatorname{tr}, \phi) \in$ $\operatorname{trace}_{\Sigma_{0}^{-}}\left(\mathcal{K}_{P}\right)$ with underlying substitution $\sigma$. Then, there exists $\left(\operatorname{tr}_{S}, \phi_{S}\right) \in \operatorname{trace}_{\Sigma_{0}^{+}}\left(\mathcal{K}_{P}\right)$ with underlying substitution $\sigma_{S}$ with $\operatorname{dom}\left(\sigma_{S}\right)=\operatorname{dom}(\sigma)$ such that $\sigma_{S}=m g u(\Gamma) \rho$, as well as two substitutions $\lambda$ and $\theta$ such that:

- $\Gamma=\left\{(u, v) \mid u, v \in E S t\left(\mathcal{K}_{P}\right)\right.$ such that $\left.u \sigma=v \sigma\right\}$.

- $\rho$ is a bijective renaming from variables in $\operatorname{dom}(\sigma) \backslash \operatorname{dom}(m g u(\Gamma))$ to constants in $\sum_{\text {fresh }}$ such that $x \rho \in \Sigma_{\text {fresh }}^{\text {atom }}$ if, and only if, $x \sigma$ is an atomic $\Sigma_{0}^{-}$-message.

- $\operatorname{dom}(\theta) \subseteq \sum_{\text {fresh }}$, for any $c \in \sum_{\text {fresh }}$ occurring in $\operatorname{tr}_{S}$, we have that $c \in \operatorname{dom}(\theta), c \theta \in \mathcal{T}\left(\Sigma, \Sigma_{0}^{-} \uplus\right.$ $\operatorname{dom}\left(\phi_{S}\right)$ ), and $\mathrm{w}^{\prime}<c$ for any $\mathrm{w}^{\prime} \in \operatorname{vars}(c \theta)$ (where $<$ is the ordering induced by $\operatorname{tr}_{S}$ ).

- for any $c \in \operatorname{dom}(\theta),(c \theta) \phi_{S} \downarrow$ is a $\Sigma_{0}^{+}$-message, and it is an atom when $c \in \sum_{\text {fresh }}^{\text {atom }}$.

- $\lambda$ is the first-order substitution associated to $\theta$ through $\phi_{S}$.

- $\phi=\phi_{S} \lambda, \sigma=\sigma_{S} \lambda$, and $\left(\operatorname{tr}_{S} \theta\right) \phi \downarrow=\operatorname{tr} \phi \downarrow$.

We say that $(\rho, \lambda, \theta)$ is a well-typed concretization of $\left(\operatorname{tr}_{S}, \phi_{S}\right)$ w.r.t. $(\operatorname{tr}, \phi)$ in the context $\mathcal{K}_{P}$ when $\rho, \lambda, \theta$ satisfy the six conditions listed above.

The fact that $\left(\operatorname{tr}_{S}, \phi_{S}\right)$ is well-typed is ensured by $\sigma_{S}=m g u(\Gamma) \rho$. Indeed, since $\Gamma$ is a set of unifiable encrypted subterms of the protocol, we know that $m g u(\Gamma)$ is well-typed (by assumption on the protocol). Hence, $\sigma_{S}$ is well-typed. Therefore, Theorem 3.8 is a direct consequence of Proposition 4.11 (the details are provided in Appendix A.3).

The proof of Proposition 4.11 is the key step for proving the existence of a well-typed trace. We provide here a detailed sketch of proof, while the full proof can be found in Appendix A.3. We omit here the subtleties between $\Sigma_{0}^{+}$and $\Sigma_{0}^{-}$. Proposition 4.11 is proved by induction. Consider an execution trace

$$
\mathcal{K}_{P} \stackrel{\operatorname{tr}}{\longrightarrow}^{*}\left(\mathcal{P} ; \phi^{-} ; \sigma^{-} ; i^{-}\right) \stackrel{\alpha}{\longrightarrow}(\mathcal{P} ; \phi ; \sigma ; i) .
$$

By induction, there exist $\phi_{S}^{-}$, and $\sigma_{S}^{-}$such that

$$
\mathcal{K}_{P} \stackrel{\operatorname{tr}_{S}^{-}}{\longrightarrow}\left(\mathcal{P} ; \phi_{S}^{-} ; \sigma_{S}^{-} ; i^{-}\right)
$$


with corresponding $\lambda^{-}$, and $\theta^{-}$as specified in the proposition. We consider the transition $\alpha$.

- The case of a phase is immediate: $\left(\operatorname{tr}_{S}^{-}, \phi_{S}^{-}\right)$can be easily extended.

- The case of an output, i.e., $\alpha=\operatorname{out}(c, \mathrm{w})$ with a corresponding process out $(c, u) . P$ ready to emit, is relatively simple. We simply need to guarantee that $u \sigma_{S}^{-}$is a message, which follows from the fact that $u \sigma^{-}$is a message and $\sigma^{-}=\sigma_{S}^{-} \lambda^{-}$.

- The difficult case is the input case: $\alpha=$ in $(c, R)$, with a corresponding process in $(c, u) . P$ ready to receive. We have that $R \phi^{-} \downarrow=u \sigma$. We are looking for $R_{S}$ such that

$$
\left(R_{S} \theta^{-}\right) \phi^{-} \downarrow=u \sigma .
$$

Such an $R_{S}$ exists, since we could take $R$. We consider the minimal $R_{S}$, w.r.t. our measure, that satisfies this property.

Step 1. We first prove that $R_{S} \phi_{S} \downarrow$ is a message: if this is not the case, we show that this contradicts the minimality of $R_{S}$ w.r.t. the measure.

Step 2. We then show that $R_{S}=C\left[R_{1}, \ldots, R_{n}\right]$ where $C$ is a context of constructors and $R_{i} \phi_{S} \downarrow$ are encrypted subterms. Indeed, if $R_{i} \phi_{S} \downarrow$ was headed by a transparent function, we could push it into the context $C$ and obtain a smaller $R_{S}$ (w.r.t. item 2 of the measure). In other words, we show that $R_{S}$ is a simple recipe.

Step 3. $R_{S}$ still does not satisfy the requirements of Proposition 4.11. It could still be a "big" recipe, which does not satisfy the relation

$$
R_{S} \phi_{S} \downarrow=u \sigma_{S}
$$

needed to pass the input action. Intuitively, we build $\overline{R_{S}}$ from $R_{S}$ by "cutting" the parts that go beyond $u \sigma_{S}$. By Lemma 4.9, we have that $\left(R_{S} \phi_{S} \downarrow\right) \lambda=u \sigma_{S} \lambda$. Assume that $R_{S} \phi_{S} \downarrow \neq u \sigma_{S}$. We know that there exists a position $p$ defined both in $R_{S} \phi_{S} \downarrow$ and $u \sigma_{S}$ where the terms differ. Moreover, since $\left(R_{S} \phi_{S} \downarrow\right) \lambda=u \sigma_{S} \lambda$, we actually have

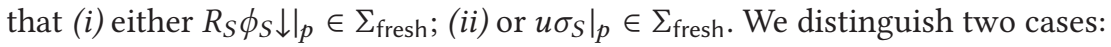

- either $p$ is a position in the context $C$, then we simply cut this part from the context $C$.

- or $p$ in under the context $C$ (inside some $\left.R_{i} \phi_{S} \downarrow\right)$. Then, $R_{i} \phi_{S} \downarrow$ is an encrypted subterm of $\phi_{S}$, which is equal to an encrypted subterm of $u \sigma_{S}$. By Lemma 4.10, they are subterms of $\Gamma$ and therefore, thanks to the application of the mgu, they are equal (thus, there is no need to "cut").

These three steps are highlighted in the detailed proof.

Example 4.12. To illustrate Step 3 of the proof sketched above, consider $\mathcal{P}=\left\{\operatorname{in}\left(c,\left\langle x_{1}, x_{2}\right\rangle\right) . P\right\}$ (here $u=\left\langle x_{1}, x_{2}\right\rangle$ ), and we assume that $x_{i} \sigma_{S}=c_{i}$ with $c_{i} \in \Sigma_{0}^{+}$(for $\left.i \in\{1,2\}\right)$. We consider the recipe $R=\left\langle\operatorname{fst}\left(\left\langle a_{1}, a_{2}\right\rangle\right)\right.$, senc $\left.\left(a_{1}, a_{2}\right)\right\rangle$, with $a_{1}, a_{2}$ in $\Sigma_{0}^{-}$. We assume that this is the first input, and thus $\theta^{-}$is the empty substitution. Clearly, we will not choose $R_{S}=R$, since $R$ is not minimal according to our measure. We will start with $R_{S}=\left\langle a_{1}\right.$, senc $\left.\left(a_{1}, a_{2}\right)\right\rangle$. We have that $R_{S} \phi_{S} \downarrow=\left\langle a_{1}, \operatorname{senc}\left(a_{1}, a_{2}\right)\right\rangle$ so it is a message, and $R_{S}$ is a simple recipe: this is consistent with Step 2. However, we have that $\left\langle x_{1}, x_{2}\right\rangle \sigma_{S}=\left\langle c_{1}, c_{2}\right\rangle$, and thus according to Step $3, R_{S}$ is too "big," and we will consider $\overline{R_{S}}=\left\langle c_{1}, c_{2}\right\rangle$ with $\theta^{+}=\left\{c_{1} \triangleright a_{1}, c_{2} \triangleright \operatorname{senc}\left(a_{1}, a_{2}\right)\right\}$.

\section{EQUIVALENCE}

We now show how to extract a well-typed witness of non-trace inclusion $\mathcal{K}_{P} \nsubseteq_{t} \mathcal{K}_{Q}$ from an arbitrary witness. Compared with the proof for reachability, the proof starts similarly: from a trace $(\operatorname{tr}, \phi) \in \mathcal{K}_{P}$, we build a well-typed trace $\left(\operatorname{tr}_{S}, \phi_{S}\right) \in \mathcal{K}_{P}$. However, an additional work is needed to 
show that such a $\operatorname{tr}_{S}$ is indeed a witness of non-trace inclusion, i.e., either there is no $\psi_{S}$ such that $\left(\operatorname{tr}_{S}, \psi_{S}\right)$ in $\mathcal{K}_{Q}$ or $\phi_{S} \nsubseteq_{S} \psi_{S}$ for any $\psi_{S}$ such that $\left(\operatorname{tr}_{S}, \psi_{S}\right) \in \operatorname{trace}_{\Sigma_{0}^{-}}\left(\mathcal{K}_{Q}\right)$.

\subsection{Some Preliminaries}

We introduce a slightly different notion of static inclusion where the attacker is also given the ability to check whether a message is atomic, i.e., a name in $\mathcal{N}$, a constant in $\Sigma_{0}^{-} \uplus \Sigma_{\text {fresh }}^{\text {atom }}$, or a constant in $\Sigma$ of sort atom. This new equivalence actually coincides with the original one.

Definition 5.1. Let $\phi_{1}$ and $\phi_{2}$ be two $\Sigma_{0}$-frames. We write $\phi_{1} \complement_{s}^{\text {atom }} \phi_{2}$ w.r.t. $\Sigma_{0}$ when $\operatorname{dom}\left(\phi_{1}\right)=$ $\operatorname{dom}\left(\phi_{2}\right)$, and:

- for any $\Sigma_{0}$-recipe $R$, we have that $R \phi_{1} \downarrow$ is a $\Sigma_{0}$-message that implies that $R \phi_{2} \downarrow$ is a $\Sigma_{0^{-}}$ message;

- for any $\Sigma_{0}$-recipe $R$, we have that $R \phi_{1} \downarrow$ is an atomic $\Sigma_{0}$-message, implies that $R \phi_{2} \downarrow$ is an atomic $\Sigma_{0}$-message; and

- for any $\Sigma_{0}$-recipes $R, R^{\prime}$ such that $R \phi_{1} \downarrow, R^{\prime} \phi_{1} \downarrow$ are $\Sigma_{0}$-messages, we have that: $R \phi_{1} \downarrow=R^{\prime} \phi_{1} \downarrow$ implies $R \phi_{2} \downarrow=R^{\prime} \phi_{2} \downarrow$.

Example 5.2. To illustrate the definition above and its difference with the original definition of static equivalence, we consider $\Sigma^{\mathrm{sign}}$ as described in Example 2.4. Let $\phi=\{\mathrm{w} \triangleright n\}$ and $\psi=\{\mathrm{w} \triangleright \operatorname{hash}(n)\}$ where $n \in \mathcal{N}$.

We have that $\phi \nsubseteq_{s}^{\text {atom }} \psi$. Indeed, we have that $w \phi \downarrow$ is an atomic message, whereas w $\psi \downarrow$ is not. Such a test is not possible when considering $\sqsubseteq_{s}$. However, relying on a non-linear rule of our rewriting system, here $\operatorname{check}(\operatorname{sign}(x, y), \mathrm{vk}(y)) \rightarrow$ ok, we can consider another test that will witness this non-inclusion, namely $\operatorname{check}(\operatorname{sign}(\mathrm{w}, \mathrm{w}), \mathrm{vk}(\mathrm{w}))=\mathrm{ok}$. Indeed, such a test holds in $\phi$ but not in $\psi$.

More generally, we can show that $\complement_{s}^{\text {atom }}$ coincides with $\sqsubseteq_{s}$.

Lemma 5.3. Let $\phi_{1}$ and $\phi_{2}$ be two $\Sigma_{0}$-frames. We have that $\phi_{1} \sqsubseteq_{s} \phi_{2}$ if, and only if, $\phi_{1} \sqsubseteq_{s}^{\text {atom }} \phi_{2}$.

Proof. The proof of the lemma relies on the fact that the attacker may always emulate the atomicity test using one of the non-linear rules, as illustrated in Example 5.2. In case $\phi_{1} \sqsubseteq_{s}^{\text {atom }} \phi_{2}$, it is easy to see that $\phi_{1} \sqsubseteq_{s} \phi_{2}$. Therefore, we consider the other implication. Let $\phi_{1}$ and $\phi_{2}$ be two $\Sigma_{0}$-frames such that $\phi_{1} \sqsubseteq_{s} \phi_{2}$; we have to establish that $\phi_{1} \complement_{s}^{\text {atom }} \phi_{2}$. Let $R$ be a $\Sigma_{0}$-recipe such that $R \phi_{1} \downarrow$ is an atomic $\Sigma_{0}$-message. We have to show that $R \phi_{2} \downarrow$ is an atomic $\Sigma_{0}$-message. By hypothesis, we know that there exists $\ell_{\text {des }} \rightarrow r_{\text {des }} \in \mathcal{R}$ with $\ell_{\text {des }}$ non-linear, i.e., several occurrences of a variable $x$ with at least one occurring at an atomic position. Let $\sigma$ be the substitution with $\operatorname{dom}(\sigma)=\operatorname{vars}\left(\ell_{\mathrm{des}}\right)$, and $\operatorname{img}(\sigma)=\{R\}$, and $R^{\prime}=\ell_{\mathrm{des}} \sigma$. We have that $R^{\prime}$ is a $\Sigma_{0}$-recipe such that $R^{\prime} \phi_{1} \downarrow$ is a $\Sigma_{0}$-message. Indeed, the rewriting rule applies, since the same atomic message occurs at each atomic position.

Relying on our hypothesis $\phi_{1} \sqsubseteq_{s} \phi_{2}$, we know that both $R \phi_{2} \downarrow$ and $R^{\prime} \phi_{2} \downarrow$ are $\Sigma_{0}$-message. In particular, we have that $\left(\ell_{\text {des }} \sigma\right) \phi_{2} \downarrow$ reduces using $\ell_{\text {des }} \rightarrow r_{\text {des }}$ meaning that atomic messages occur at each atomic position, and thus $R \phi_{2} \downarrow$ is an atomic $\Sigma_{0}$-message.

Note that this lemma crucially relies on our assumption that our set of rewriting rules contains at least one non-linear rule.

Our measure. We extend our measure to tests, as considered for checking static inclusion. Formally, a test $T$ is either a single recipe $R$, or a pair $R, R^{\prime}$ of two recipes. In such a case, i.e., when $T$ is a pair $R, R^{\prime}$, we define:

$$
\mu_{\phi_{S}}(T)=\left(\mu_{\phi_{S}}^{1}(R) \uplus \mu_{\phi_{S}}^{1}\left(R^{\prime}\right), \mu_{\phi_{S}}^{2}(R)+\mu_{\phi_{S}}^{2}\left(R^{\prime}\right), \mu^{3}(R)+\mu^{3}\left(R^{\prime}\right)\right) .
$$




\subsection{Completeness}

The equivalence counterpart of Proposition 4.11 is the following proposition, which states that we can transform a witness of non-trace inclusion into a well-typed one.

Proposition 5.4. Let $\mathcal{K}_{P}$ and $\mathcal{K}_{Q}$ be two initial $\Sigma_{0}^{-}$-configurations such that $\mathcal{K}_{P} \nsubseteq_{t} \mathcal{K}_{Q}$ w.r.t. $\Sigma_{0}^{-}$. Let $(\operatorname{tr}, \phi) \in \operatorname{trace}_{\Sigma_{0}^{-}}\left(\mathcal{K}_{P}\right)$ with underlying substitution $\sigma$ be a witness of non-inclusion. Then, there exists $\left(\operatorname{tr}_{S}, \phi_{S}\right) \in$ trace $_{\Sigma_{0}^{+}}\left(\mathcal{K}_{P}\right)$ a witness of this non-inclusion with underlying substitution $\sigma_{S}$ such that $\sigma_{S}=m g u(\Gamma) \rho$, as well as two substitutions $\lambda_{P}$ and $\theta$ such that $\left(\rho, \lambda_{P}, \theta\right)$ is a well-typed concretization of $\left(\operatorname{tr}_{S}, \phi_{S}\right)$ w.r.t. $(\operatorname{tr}, \phi)$ in the context $\mathcal{K}_{P}$, as defined in Proposition 4.11.

Exactly like for the reachability case, the fact that $\left(\operatorname{tr}_{S}, \phi_{S}\right)$ is well-typed is ensured by $\sigma_{S}=$ $m g u(\Gamma) \rho$. Indeed, since $\Gamma$ is a set of unifiable encrypted subterms of $\mathcal{K}_{P}$, we know that $m g u(\Gamma)$ is well-typed (by assumption on the protocol). Hence, $\sigma_{S}$ is well-typed. Therefore, Theorem 3.9 is a direct consequence of Proposition 5.4 (the details are provided in Appendix B).

We provide here a detailed sketch of proof of Proposition 5.4, leaving the full proof in Appendix B. Consider $(\operatorname{tr}, \phi) \in \operatorname{trace}_{\Sigma_{0}^{-}}\left(\mathcal{K}_{P}\right)$ with underlying substitution $\sigma$ be a witness of non-inclusion. We explain how to build a well-typed witness of non-inclusion. Thanks to Proposition 4.11, there exists a well-typed trace $\left(\operatorname{tr}_{S}, \phi_{S}\right) \in \operatorname{trace}_{\Sigma_{0}^{+}}\left(\mathcal{K}_{P}\right)$ with underlying substitution $\sigma_{S}$ such that $\sigma_{S}=m g u(\Gamma) \rho$, and $\lambda_{P}$ and $\theta$ satisfying the conditions of Proposition 4.11. We would like to show that $\left(\operatorname{tr}_{S}, \phi_{S}\right)$ is a witness of non-inclusion. There must exist a trace $\left(\operatorname{tr}_{S}, \psi_{S}\right) \in \operatorname{trace}_{\Sigma_{0}^{+}}\left(\mathcal{K}_{Q}\right)$ such that $\phi_{S} \sqsubseteq_{s} \psi_{S}$; otherwise, we can already conclude that $\left(\operatorname{tr}_{S}, \phi_{S}\right)$ is a witness of non-inclusion. Then the first step of the proof consists in showing that we can instantiate $\left(\operatorname{tr}_{S}, \psi_{S}\right)$ by $\theta$ and $\lambda_{Q}$ such that $\psi=\psi_{S} \lambda_{Q}, \operatorname{tr} \psi \downarrow=\left(\operatorname{tr}_{S} \theta\right) \psi \downarrow$, and $(\operatorname{tr}, \psi) \in \operatorname{trace}_{\Sigma_{0}^{-}}\left(\mathcal{K}_{Q}\right)$. We also show that $\psi$ satisfies the assumptions of Lemmas 4.8 and 4.9 .

In a second step of the proof, we show that $\phi_{S} \sqsubseteq_{s} \psi_{S}$ implies $\phi \sqsubseteq_{s} \psi$, hence a contradiction. More specifically, we show by induction on $\mu_{\phi_{S}}(T)$, that:

for any test $T$, if $T \theta$ holds for $\phi$, then it holds for $\psi$.

This allows us to conclude that any test $T$ that holds in $\phi$ also holds in $\psi$, since we may simply consider tests without constants in $\operatorname{dom}(\theta)$. Hence, $\phi \sqsubseteq_{s} \psi$.

We consider all the tests defined by the static inclusion $\bigsqcup_{s}^{\text {atom }}$. In particular, the attacker can test directly whether a message is an atom or not, which avoids to consider a "big" recipe of the form $\operatorname{check}(\operatorname{sign}(R, R), \mathrm{vk}(R))=$ ok instead of simply $R$. We show $\left(^{*}\right)$ by induction on our measure. Therefore, let us assume that $\left(^{*}\right)$ holds for any test $T^{\prime}$ such that $\mu_{\phi_{S}}\left(T^{\prime}\right)<\mu_{\phi_{S}}(T)$. We consider the three possible cases for $T$.

- Either $T$ checks whether the term induced by $R$ is a message. We need to show that $(R \theta) \phi \downarrow$ is a message that implies $(R \theta) \psi \downarrow$ is a message. If $R \phi_{S} \downarrow$ is a message, then so is $R \psi_{S} \downarrow$ by static inclusion, and we can rather easily conclude that $(R \theta) \psi \downarrow$ is a message. If $R \phi_{S} \downarrow$ is not a message, similarly to the reachability case, we can build smaller tests, apply the induction hypothesis, and reconstruct $R$. An additional difficulty comes from the fact that we now also need to transfer the properties on $\psi$.

- Or $T$ checks whether the term induced by $R$ is atomic. We need to show that $(R \theta) \phi \downarrow$ is atomic implies $(R \theta) \psi \downarrow$ is atomic. Thanks to the previous case, we already know that $(R \theta) \psi \downarrow$ is a message. Actually, $R \phi_{S} \downarrow$ must be atomic, because $(R \theta) \phi \downarrow=R \phi_{S} \downarrow \lambda_{P}$ is atomic and due to the constraints on $\lambda_{P}$. We deduce that $R \psi_{S} \downarrow$ is atomic by static inclusion, and thus $(R \theta) \psi \downarrow=$ $R \psi_{S} \downarrow \lambda_{Q}$ is atomic, since $\lambda_{Q}$ preserves atomicity.

- Or $T$ is an equality test $R=R^{\prime}$. If $R$ and $R^{\prime}$ are headed by the same constructor symbol, we may simply open each recipe and apply the induction hypothesis. Therefore, we may 
assume that $R=C\left[R_{1}, \ldots, R_{n}\right]$ and $R^{\prime}$ is headed by a destructor. As for the reachability case, we can show that the $R_{i}$ as well as $R^{\prime}$ are subterm recipes yielding an encrypted subterm. We have that $R \phi_{S} \downarrow$ and $R^{\prime} \phi_{S} \downarrow$ are messages. Either $R \phi_{S} \downarrow=R^{\prime} \phi_{S} \downarrow$. Then this holds for $\psi_{S}$ as well and therefore for $\psi$. Or $R \phi_{S} \downarrow \neq R^{\prime} \phi_{S} \downarrow$ and yet $\left(R \phi_{S}\right) \downarrow \lambda_{P}=\left(R^{\prime} \phi_{S}\right) \downarrow \lambda_{P}$. Let us consider a leaf position $p$ on which the two terms differ, i.e., $p$ exits in both $R \phi_{S} \downarrow$ and $R^{\prime} \phi_{S}$, but $R \phi_{S} \downarrow_{p} \neq\left. R^{\prime} \phi_{S} \downarrow\right|_{p}$. We assume that $p$ is a leaf of $R^{\prime} \phi_{S} \downarrow$, and since $\lambda_{P}$ makes these two terms equal, we know that $R^{\prime} \phi_{S} \downarrow=c \in \sum_{\text {fresh }}$ (the case where $p$ is a leaf of $R \phi_{S}$ is actually simpler). - either $p$ belongs to one of the $R_{i} \phi_{S} \downarrow$. Then, as for the reachability case, $R_{i} \phi_{S} \downarrow$ is an encrypted subterm of $\phi_{S}$, which is equal to an encrypted subterm of $R^{\prime} \phi_{S} \downarrow$, and thus an encrypted subterm of $\phi_{S}$, since $R^{\prime}$ is a subterm recipe. By Lemma 4.10, they are subterms of $\Gamma$ and therefore, thanks to the application of the mgu, they are equal.

- or $p$ belongs to the context $C$, then we build $\bar{C}$ from $C$ by replacing $\left.C\right|_{p}$ by $c$ for all such $c$. Consider the corresponding recipe $\bar{R}=\bar{C}\left[R_{1}, \ldots, R_{n}\right]$. We show that the equality $\bar{R}=R^{\prime}$ holds in $\phi_{S}$, since we have removed all the differences. Therefore, we have that $\bar{R}=R^{\prime}$ holds in $\psi_{S}$. Moreover, the equality $\bar{R}=R$ holds relying on our induction hypothesis.

\section{DEALING WITH REPLICATED PROCESSES}

In this section, we consider the case of processes with replication; that is, processes that can be executed an arbitrary number of times. We first extend our process algebra (syntax and semantics). Then, we explain how the type-compliance hypothesis can be effectively checked on replicated processes. Finally, we show how to extend the results stated in Section 3 to this new setting.

\subsection{Syntax and Semantics}

When considering replicated processes, it becomes useful to add a contruct to generate fresh names. Therefore, in addition to the replication operator, we consider two additional instructions allowing us to generate fresh basic names and fresh channel names. For this purpose, we introduce the set $C h_{\text {fresh }}$ of fresh channels. Those channel names-as every channel name we have considered so far-are public. Then, we extend our process algebra as follows:

$$
P, Q:=\ldots \mid \text { new } n . P \mid \text { new } c^{\prime} \text {.out }\left(c, c^{\prime}\right) . P \mid ! P,
$$

where $n \in \mathcal{N}, c \in C h \uplus C h_{\text {fresh }}$ and $c^{\prime} \in C h_{\text {fresh }}$.

We extend our semantics with the following 3 rules:

$$
\begin{array}{rllr}
(i: \text { new } n . P \uplus \mathcal{P} ; \phi ; \sigma ; i) & \stackrel{\tau}{\longrightarrow} & (i: P\{m / n\} ; \phi ; \sigma ; i) & \text { with } m \in \mathcal{N} \text { fresh. } \\
\left(i: \text { new } c^{\prime} \text {.out }\left(c, c^{\prime}\right) . P \uplus \mathcal{P} ; \phi ; \sigma ; i\right) & \stackrel{\text { out }\left(c, c^{\prime \prime}\right)}{\longrightarrow} & \left(i: P\left\{c^{\prime \prime} / c^{\prime}\right\} \uplus \mathcal{P} ; \phi ; \sigma ; i\right) & \text { with } c^{\prime \prime} \text { a fresh channel. } \\
(i: ! P \uplus \mathcal{P} ; \phi ; \sigma ; i) & \stackrel{\tau}{\longrightarrow} & \left(i: P^{\prime} \uplus i: ! P \uplus \mathcal{P} ; \phi ; \sigma ; i\right) & \text { with } P^{\prime} \text { a copy of } P \\
& & & \text { where variables bound in the inputs are } \alpha \text {-renamed. }
\end{array}
$$

The construction new $n . P$ generated a fresh name $m$ and replaces $n$ by $m$ in $P$. The public channel creation instruction new $c^{\prime}$.out $\left(c, c^{\prime}\right) . P$ creates a fresh channel $c^{\prime \prime}$, and replaces $c^{\prime}$ by $c^{\prime \prime}$ in $P$. The channel name $c^{\prime \prime}$ is not added into the frame. Actually, all the channel names are assumed to be public and the label out $\left(c, c^{\prime \prime}\right)$ on the arrow makes this instruction observable. Finally, $! P$ allows us to launch a new copy of $P$, and thus consider processes that can be executed an arbitrary number of times.

First, we may note that our typing result stated in Section 3 still apply when extending our process algebra with the two constructions allowing one to generate fresh basic and channel names. We did not include these instructions in our initial model, since they are mostly useless in a model without replication. In the remaining, we admit Theorems 3.8 and 3.9 for this simple extension, 
and we show how to add the replication operator. For this very simple extension, the main point is to assume that the typing system $(\mathcal{T}, \delta)$ under study respects the name binding; that is:

$$
(i \text { : new } n . P \uplus \mathcal{P} ; \phi ; \sigma ; i) \stackrel{\tau}{\longrightarrow}(i: P\{m / n\} \uplus \mathcal{P} ; \phi ; \sigma ; i) \quad \text { with } m \in \mathcal{N} \text { fresh and } \delta(n)=\delta(m)
$$

Now, we go back to our replication operator. We say that a process $P^{\prime}$ is a finite version of $P$ if $P^{\prime}$ is $P$, except that each subprocess of the form $! Q$ occurring in $P$ is replaced with $\left(Q_{1}|\cdots| Q_{n}\right)$ (for some $n \geq 0$ ) where each $Q_{i}$ is a copy of $Q$ (in which bound variables are $\alpha$-renamed). In presence of nested replications, the transformation may be applied several times until obtaining a process without replication. This notion naturally extends to multisets of processes and to configurations. Intuitively, a finite version represents a version of the process that can only be executed a fixed number of times. We define the $k$-unfolding $\operatorname{unfold}_{k}(P)$ of the replicated process $P$ as a finite version of $P$ such that each replication has been unfolded exactly $k$ times. For instance, we have that unfold ${ }_{1}(P)$ is the process obtained from $P$ by simply removing the ! operator, whereas unfold ${ }_{0}(P)$ is the process obtained from $P$ by removing parts of the process under a replication.

\subsection{Type Compliance}

We now consider a process $P$ in our extended syntax (i.e., with replication), and a typing system $(\mathcal{T}, \delta)$. We assume that $\delta(m)=\delta(n)$ whenever $m \in \mathcal{N}$ instantiates the name $n$, and $\delta(x)=\delta(y)$ when $x$ and $y$ are an $\alpha$-renaming of the same variable due to replication. We now establish the following result:

If unfold ${ }_{2}(\mathcal{P})$ is type compliant w.r.t. $(\mathcal{T}, \delta)$, then any finite version of $\mathcal{P}$ is type compliant w.r.t. $(\mathcal{T}, \delta)$, and we say that $\mathcal{P}$ is type-compliant w.r.t. $(\mathcal{T}, \delta)$.

Sketch of Proof. Let $\mathcal{P}^{\prime}$ be a finite version of $\mathcal{P}$, and $t_{1}, t_{2} \in E S t\left(\mathcal{P}^{\prime}\right)$ be two unifiable terms. We have to prove that $\delta\left(t_{1}\right)=\delta\left(t_{2}\right)$. Without loss of generality, we may assume that we are in one of the two following cases:

(1) In $\mathcal{P}$, the term $t_{1}$ occurs in a prefix of the process where the term $t_{2}$ occurs.

(2) There is a $\left(Q_{1}|\cdots| Q_{n}\right)$ in $\mathcal{P}^{\prime}$ such that $t_{1}$ occurs in $Q_{1}$ and $t_{2}$ occurs in $Q_{2}$.

In the first case, let $Q \in \mathcal{P}$ be the process where $t_{2}$ occurs. We have that $t_{1}$ also occurs in $Q$. So both $t_{1}$ and $t_{2}$ occur in $Q$, so $t_{1}$ and $t_{2}$ occur in unfold $(Q)$, and thus in $\operatorname{unfold}_{2}(\mathcal{P})$. As unfold $(\mathcal{P})$ is type-compliant, we have $\delta\left(t_{1}\right)=\delta\left(t_{2}\right)$.

In the second case, we distinguish two cases:

- either $\left(Q_{1}|\cdots| Q_{n}\right)$ is a subprocess of $\mathcal{P}$ and thus $t_{1}, t_{2}$ occur in $\mathcal{P}$ and thus in $\operatorname{unfold}_{2}(\mathcal{P})$. By hypothesis, we thus have $\delta\left(t_{1}\right)=\delta\left(t_{2}\right)$, and this allows us to conclude.

- or $\left(Q_{1}|\cdots| Q_{n}\right)$ is coming from a !Q, and up to a (well-typed) $\alpha$-renaming of variables, $\left(Q_{1} \mid\right.$ $\left.Q_{2}\right)=$ unfold $_{2}(! Q)$. Therefore, we have that $t_{1}$ and $t_{2}$ are encrypted subterms of unfold $2(! Q)$, and thus of unfold ${ }_{2}(\mathcal{P})$. We get $\delta\left(t_{1}\right)=\delta\left(t_{2}\right)$, and we conclude.

\subsection{Typing Result for Reachability}

Now, we explain how to derive a typing result for reachability for processes with replication. More precisely, we establish the following statement:

Let $\mathcal{K}_{P}$ be a $\Sigma_{0}^{-}$-configuration type-compliant w.r.t. $\left(\mathcal{T}_{0}, \delta_{0}\right)$. If $\mathcal{K}_{P} \stackrel{\operatorname{tr}^{\prime}}{\Longrightarrow}(\mathcal{P} ; \phi ; \sigma ; i)$ w.r.t. $\Sigma_{0}^{-}$, then there exists a well-typed execution $\mathcal{K}_{P} \stackrel{\operatorname{tr}^{\prime}}{\Longrightarrow}\left(\mathcal{P} ; \phi^{\prime} ; \sigma^{\prime} ; i\right)$ w.r.t. $\Sigma_{0}^{+}$ such that $\overline{\operatorname{tr}^{\prime}}=\overline{\operatorname{tr}}$. 
Remember that $\overline{\operatorname{tr}}$ is obtained from tr by replacing any action in $(c, R)$ by in $\left(c,{ }_{-}\right)$, any out $(c, \mathrm{w})$ by out $\left(c,{ }_{-}\right)$, while keeping the other actions (i.e., phase $i$ and out $\left.\left(c, c^{\prime \prime}\right)\right)$ unchanged.

First, we make the observation that every attack is an attack against a finite execution (Fact 1). Then, we establish that any execution of a finite version is an execution of the original process (Fact 2). These properties correspond to expected behaviors when considering finite versions of a process.

FACT 1. If there is a trace $(\operatorname{tr}, \phi)$ of $\mathcal{P}$ of length at most $\ell$, then $(\operatorname{tr}, \phi)$ is a trace of $\operatorname{unfold}_{\ell}(\mathcal{P})$.

Sketch of Proof. We proceed by induction on $\ell$. When $\ell=0$, the result is obvious. Now, assume that $\operatorname{tr}=\operatorname{tr}_{0} . \alpha$ for some observable action $\alpha$. Since the induction hypothesis applies on $\operatorname{tr}_{0}$, we know that the execution corresponding to $\operatorname{tr}_{0}$ can be done starting from unfold $\boldsymbol{l}_{\ell-1}(\mathcal{P})$. Then either $\operatorname{tr}$ is a trace of unfold $\boldsymbol{d}_{\ell}(\mathcal{P})$, or there exists a replication in $\mathcal{P}$ and some extra unfoldings are necessary to execute $\alpha$. Actually, one supplementary unfolding will be enough. Hence, the result.

FACT 2. Let $\mathcal{P}^{\prime}$ be a finite version of some process $\mathcal{P}$ with replication. If $(\operatorname{tr}, \phi)$ is a trace of $\mathcal{P}^{\prime}$, then it is also a trace of $\mathcal{P}$.

Sketch of Proof. To mimic the execution tr starting from $\mathcal{P}$ (with replication), we will simply unfold the replications when needed, inserting some $\tau$ (unobservable actions) in the trace tr.

Now, we are able to establish our typing result for reachability in presence of replicated processes. Assuming we have a trace tr starting from some initial configuration $\mathcal{K}_{P}=(\mathcal{P} ; \emptyset ; \emptyset ; 0)$. Fact 1 tells us that this trace can be performed starting from $\mathcal{K}_{P}^{\prime}=\left(\mathcal{P}^{\prime} ; \emptyset ; \emptyset ; 0\right)$ where $\mathcal{P}^{\prime}=$ unfold $_{\ell}(\mathcal{P})$. Now, Theorem 3.8 (more precisely its simple extension discussed in Section 6.1) applies. Note that type-compliance is satisfied by any finite version of $\mathcal{P}$ and thus in particular unfold $_{\ell}(\mathcal{P})$ is type-compliant. Therefore, there exists a well-typed trace $\operatorname{tr}^{\prime}$ of $\mathcal{K}_{P}^{\prime}$ such that $\overline{\operatorname{tr}^{\prime}}=\overline{\operatorname{tr}}$, and Fact 2 allows us to ensure that the trace $\operatorname{tr}^{\prime}$ can be performed starting from the initial configuration $\mathcal{K}_{P}$. This proves the result.

\subsection{Equivalence Result}

In this section, we explain how to establish the typing result regarding equivalence. We aim at establishing the following statement.

Let $\mathcal{K}_{P}$ be a $\Sigma_{0}^{-}$-configuration type-compliant w.r.t. $\left(\mathcal{T}_{0}, \delta_{0}\right)$ and $\mathcal{K}_{Q}$ a $\Sigma_{0}^{-}$configuration. If $\mathcal{K}_{P} \nsubseteq_{t} \mathcal{K}_{Q}$ w.r.t. $\Sigma_{0}^{-}$, then there exists a witness $(\operatorname{tr}, \phi) \in$ trace $_{\Sigma_{0}^{+}}\left(\mathcal{K}_{P}\right)$ of this non-inclusion such that its underlying execution $\mathcal{K}_{P} \stackrel{\text { tr }}{\Longrightarrow}$ $(\mathcal{P} ; \phi ; \sigma ; i)$ w.r.t. $\Sigma_{0}^{+}$is well-typed.

Sketch of Proof. Let $(\operatorname{tr}, \phi)$ be a witness of $\mathcal{K}_{P} \nsubseteq_{t} \mathcal{K}_{Q}$ w.r.t. $\Sigma_{0}^{-}$and we denote $\ell$ the length of tr. Fact 1 tells us that there is a finite version $\mathcal{K}_{P}^{\prime}$ of $\mathcal{K}_{P}$ (the $\ell^{\text {th }}$ unfolding of the process is actually a good candidate) such that $(\operatorname{tr}, \phi)$ is a trace of $\mathcal{K}_{P}^{\prime}$. If there was a $\psi$ such that $(\operatorname{tr}, \psi)$ is a trace of unfold ${ }_{\ell}\left(\mathcal{K}_{Q}\right)$, then by Fact 2 , ( $\left.\operatorname{tr}, \psi\right)$ would be a trace of $\mathcal{K}_{Q}$, and thus $\phi \nsubseteq_{s} \psi$. So the trace $(\operatorname{tr}, \phi)$ is a trace of non-inclusion for $\mathcal{K}_{P}^{\prime} \nsubseteq$ unfold $\ell\left(\mathcal{K}_{Q}\right)$.

By Theorem 3.9, there is a well-typed trace $\left(\operatorname{tr}^{\prime}, \phi^{\prime}\right)$ of this non-inclusion (because $\mathcal{K}_{P}^{\prime}$ is type compliant and $\mathcal{K}_{P}^{\prime}$ and unfold ${ }_{\ell}\left(\mathcal{K}_{Q}\right)$ are processes without replication). By Fact $2,\left(\operatorname{tr}^{\prime}, \phi^{\prime}\right)$ is a trace of $\mathcal{K}_{P}$. Now, it only remains to prove that $\operatorname{tr}^{\prime}$ is a trace of non-inclusion for $\mathcal{K}_{P} \nsubseteq_{t} \mathcal{K}_{Q}$. Thanks to Fact 1 and since $\operatorname{tr}$ and $\operatorname{tr}^{\prime}$ have the same length $\left(\mathrm{as} \overline{\operatorname{tr}^{\prime}}=\overline{\operatorname{tr}}\right)$ if there is a $\psi^{\prime}$ such that $\left(\operatorname{tr}^{\prime}, \psi^{\prime}\right)$ of $\mathcal{K}_{Q}$, then $\left(\operatorname{tr}^{\prime}, \psi^{\prime}\right)$ is also a trace of $\operatorname{unfold}\left(\mathcal{K}_{Q}\right)$. As $\left(\operatorname{tr}^{\prime}, \phi^{\prime}\right)$ is a witness of non-inclusion for 
$\mathcal{K}_{P}^{\prime} \nsubseteq$ unfold $_{\ell}\left(\mathcal{K}_{Q}\right)$, we have that $\phi^{\prime} \nsubseteq_{s} \psi^{\prime}$. This allows us to conclude that $\left(\operatorname{tr}^{\prime}, \phi^{\prime}\right)$ is indeed a witness of non-inclusion for $\mathcal{K}_{P} \nsubseteq_{t} \mathcal{K}_{Q}$.

\section{EXAMPLES}

We review several protocols of the literature and identify whether our main results can be applied; that is, we identify when the protocols satisfy the type-compliance condition. Our small attack result is particularly interesting when it is established w.r.t. a structure-preserving typing system. Indeed, in such a case, the typing result allows one to bound the size of the messages involved in the smallest witness. Therefore, in this section, we aim at establishing type-compliance w.r.t. a structure-preserving typing system. We first discuss which scenario is considered.

\subsection{Complete Scenario}

We explain which scenario and which security property we consider, illustrated on the NSL protocol. As presented in Example 2.5 and Example 2.12, we may need to consider a rich scenario when searching for attacks. In the case of a 2-agent protocol, like the Needham-Schroeder-Lowe protocol, we wish to instantiate the roles $P_{A}$ and $P_{B}$ with different combinations of honest and dishonest agents. We consider $\mathrm{a}$ and $\mathrm{b}$ to be two honest agents, whereas $\mathrm{c}$ is dishonest, and denote by $P_{A}\left(z_{A}, z_{B}\right)$ (resp. $\left.P_{B}\left(z_{B}, z_{A}\right)\right)$ the role $P_{A}\left(\right.$ resp. $\left.P_{B}\right)$ played by agents $z_{A}$ and $z_{B}$. Then, we consider the following:

$$
P_{\mathrm{NSL}}^{\mathrm{semi}}=P_{A}(\mathrm{a}, \mathrm{b})\left|P_{B}(\mathrm{~b}, \mathrm{a})\right| P_{A}(\mathrm{a}, \mathrm{c})\left|P_{B}(\mathrm{~b}, \mathrm{c})\right| P_{A}(\mathrm{~b}, \mathrm{a})\left|P_{B}(\mathrm{a}, \mathrm{b})\right| P_{A}(\mathrm{~b}, \mathrm{c}) \mid P_{B}(\mathrm{a}, \mathrm{c}) .
$$

$P_{\mathrm{NSL}}^{\text {semi }}$ considers all possible combinations of a, b, c within $P_{A}$ and $P_{B}$, except that we exclude the processes $P_{A}(\mathrm{c}, \mathrm{a})$ and $P_{A}(\mathrm{c}, \mathrm{b})$ as well as $P_{B}(\mathrm{c}, \mathrm{b})$ and $P_{B}(\mathrm{c}, \mathrm{a})$. Indeed, these four processes describe the behavior of a dishonest agent $\mathrm{c}$, which is already included within the semantics of our model. $P_{\mathrm{NSL}}^{\mathrm{semi}}$ models the possible two-way interactions between honest agents, and between honest agents willing to establish a session with an attacker. Note that processes $P_{A}(\mathrm{a}, \mathrm{b}), P_{B}(\mathrm{a}, \mathrm{b})$, and $P_{B}(\mathrm{a}, \mathrm{c})$ correspond respectively to $P_{A}, P_{B}$, and $P_{B}^{\prime}$ in Example 2.5. $P_{\mathrm{NSL}}^{\mathrm{semi}}$ models a complete scenario with one session only of each possible instantiation of a role. As explained in Section 6, type-compliance and well-typedness can be easily lifted to protocols with replication. In a slightly different setting, Reference [23] has established that type compliance is guaranteed for an unbounded number of sessions as soon as a protocol is type-compliant when 2 sessions are considered for each role and each possible choice of agents. For this reason, we consider a complete protocol $P_{\mathrm{NSL}}^{\text {complete }}=P_{\mathrm{NSL}}^{\text {semi }}$ $P_{\mathrm{NSL}}^{\prime s e m i}$ where $P_{\mathrm{NS}}^{\prime \text { semi }}$ is a copy of $P_{\mathrm{NSL}}^{\text {semi }}$ alpha-renamed to ensure names and variables are all distinct in $P_{\mathrm{NSL}}^{\mathrm{complete}}$, and the same type is given to names and variables that have been alpha-renamed. This scenario is complete in the sense that, according to the approach of Reference [23], a richer scenario derived from this one by adding more copies of a process will still be type-compliant according to our definition (note that no more encrypted subterms will be introduced).

For the security property, we consider the secrecy of a key or a nonce, where secrecy is encoded as a combination of key usability and "which key-concealing." For NSL, we consider secrecy of the nonce $n_{b}$ as received by $P_{A}(\mathrm{a}, \mathrm{b})$. To that end, we consider two variants of $P_{A}(\mathrm{a}, \mathrm{b})$ : in the first one, we add a final action out $\left(c_{A}, \operatorname{senc}\left(m_{1}, n_{b}\right)\right)$, whereas we add out $\left(c_{A}, \operatorname{senc}\left(m_{2}, k\right)\right)$ in the second, where $k$ is a fresh nonce, and $m_{1}$ and $m_{2}$ are two publicly known constants. However, for NSL, the resulting protocols are not type-compliant. Indeed, there is an encrypted subterm aenc $\left(x_{1}, \operatorname{pub}\left(s k_{b}\right), r_{3}\right)$ in $P_{A}$ (Example 2.5) that can be unified with another encrypted subterm from the protocol. Note that the scenario we consider is richer than the scenario from Example 3.7, which explains why the typing system considered is not enough to make the entire protocol typecompliant. To ensure type-compliance, we need here to consider a tagged version of the protocol 


\begin{tabular}{|l|c|c|}
\hline Needham-Schroeder-Lowe & $\begin{array}{c}\text { Type-compliance } \\
\text { with tuples }\end{array}$ & $\begin{array}{c}\text { Type-compliance } \\
\text { with tagging }\end{array}$ \\
Wide Mouth Frog & $\times$ & $\checkmark$ \\
Denning Sacco with shared keys & $\checkmark$ & $\checkmark$ \\
Needham-Schroeder with shared keys & $\checkmark$ & $\checkmark$ \\
Yahalom-Lowe & $\checkmark$ & $\checkmark$ \\
Yahalom-Paulson & $\times$ & $\checkmark$ \\
Otway-Rees & $\checkmark$ & $\checkmark$ \\
Denning Sacco with signature & $\checkmark$ & $\checkmark$ \\
Passive authentication & $\checkmark$ & $\checkmark$ \\
Active authentication & $\checkmark$ & $\checkmark$ \\
\hline
\end{tabular}

Fig. 3. Type-compliance of protocols for Sections 7.2 and 7.3.

where a public constant is appended to each plaintext in the specification. The informal specification is given below.

$$
\begin{array}{ll}
A \rightarrow B: & \left\{1, N_{a}, A\right\}_{\operatorname{pub}(B)}, \\
B \rightarrow A: & \left\{2, N_{a}, N_{b}, B\right\}_{\operatorname{pub}(A)}, \\
A \rightarrow B: & \left\{3, N_{b}\right\}_{\operatorname{pub}(B)}
\end{array}
$$

\subsection{Review of Key-exchange Protocols}

We consider several protocols from the literature and investigate their type-compliance for the complete scenario. As well as for the Needham-Schroeder-Lowe protocol, the security property we consider is the secrecy of secret (nonce or session key) exchanged between two honest agents a and b, encoded as a combination of key usability and "which key-concealing." Tuples are encoded directly, without using nested pairs.

The complete scenario for a 3-party protocol with a trusted server $S$ is somewhat more complex than for a 2-party protocol. We need to consider a server $S$ willing to establish a session between all pairs of agents in $\{a, b, c\}$, in addition of the usual interactions between $a, b$, and c. Each of the protocols is thus modeled as 14 processes, leading to 28 processes after duplication.

Type-compliance of the protocols has been verified automatically. In some cases, we needed to tag the protocol to ensure type compliance, as for the case of the NSL protocol. Our findings are summarized in Figure 3, which describes which protocol is type-compliant, with or without tags. We discuss below each protocol individually.

Wide Mouth Frog. The Wide Mouth Frog protocol can be informally described as follows.

$$
\begin{array}{ll}
A \rightarrow S: & A,\left\{B, K_{a b}\right\}_{K_{a s}}, \\
S \rightarrow B: & \left\{A, K_{a b}\right\}_{K_{b s}} .
\end{array}
$$

Here, we verify the strong secrecy of $K_{a b}$ as received by $B$. We consider a structure-preserving typing system that associates the type agent to agents a, b, c, and $S$, the type session to negotiated keys between the agents such as $K_{a b}$, and the type longterm to long-term keys $K_{a s}, K_{b s}$, and $K_{c s}$. The constants $m_{1}$ and $m_{2}$ occurring in the security property (as for the NSL protocol) are typed with a type constant. This protocol is type-compliant, without requiring any additional tagging, as every encrypted subterm is of the form senc(〈agent, session〉, longterm), except for the terms that come from the encoding of the security property, which use a constant as plaintext and thus cannot be unified with other ciphertext. 
Denning Sacco with shared keys. The Denning Sacco protocol can be informally described as follows.

$$
\begin{array}{ll}
A \rightarrow S: & A, B, \\
S \rightarrow A: & \left\{B, K_{a b},\left\{K_{a b}, A\right\}_{K_{b s}}\right\}_{K_{a s}}, \\
A \rightarrow B: & \left\{K_{a b}, A\right\}_{K_{b s} .}
\end{array}
$$

We consider the same typing system as for the Wide Mouth Frog protocol. This protocol is typecompliant, without requiring any additional tagging. The difference in the arity of the plaintexts in the protocol specification ensures all possible unifications between encrypted subterms occur between encrypted subterms of the same type.

Needham-Schroeder with shared keys. The Needham-Schroeder symmetric key protocol can be informally described as follows.

$$
\begin{array}{ll}
A \rightarrow S: & A, B, N_{a}, \\
S \rightarrow A: & \left\{B, N_{a}, K_{a b},\left\{A, K_{a b}\right\} K_{b s}\right\}_{K_{a s}}, \\
A \rightarrow B: & \left\{A, K_{a b}\right\}_{K_{b s}}, \\
B \rightarrow A: & \left\{\text { req, } N_{b}\right\}_{K_{a b}}, \\
A \rightarrow B: & \left\{\text { rep, } N_{b}\right\}_{K_{a b}} .
\end{array}
$$

This protocol is type-compliant, without requiring any additional tagging, using the same structure-preserving typing system as before.

Yahalom-Lowe. The Yahalom-Lowe protocol can be informally described as follows:

$$
\begin{array}{ll}
A \rightarrow B: & A, N_{a}, \\
B \rightarrow S: & \left\{A, N_{a}, N_{b}\right\}_{K_{b s}}, \\
S \rightarrow A: & \left\{B, K_{a b}, N_{a}, N_{b}\right\}_{K_{a s}}, \\
S \rightarrow B: & \left\{A, K_{a b}\right\}_{K_{b s},}, \\
A \rightarrow B: & \left\{A, B, S, N_{b}\right\}_{K_{a b}} .
\end{array}
$$

This protocol is type-compliant, without requiring any additional tagging, using the same structure-preserving typing system as before. Note that type-compliance depends on the encoding of tuples: When considered as nested pairs, type-compliance is not guaranteed, as more encrypted subterms become unifiable.

Yahalom-Paulson. The Yahalom-Paulson protocol can be informally described as follows:

$$
\begin{array}{ll}
A \rightarrow B: & A, N_{a}, \\
B \rightarrow S: & B, N_{b},\left\{A, N_{a}\right\}_{K_{b s}}, \\
S \rightarrow A: & N_{b},\left\{B, K_{a b}, N_{a}\right\}_{K_{a s}},\left\{A, B, K_{a b}, N_{b}\right\}_{K_{b s}}, \\
A \rightarrow B: & \left\{A, B, K_{a b}, N_{b}\right\}_{K_{b s}},\left\{N_{b}\right\}_{K_{a b}} .
\end{array}
$$

This protocol is not type-compliant. Indeed its formal specification contains a term $\operatorname{senc}\left(x_{n b}, x_{K a b}\right)$, in the encrypted subterms of $A$, which can be unified with any other encrypted subterm from the protocol, such as subterms encrypted with long-term keys $K_{a s}$ and $K_{b s}$. To ensure type-compliance with a non-trivial typing system, we consider a tagged version of the protocol:

$$
\begin{array}{ll}
A \rightarrow B: & A, N_{a}, \\
B \rightarrow S: & B, N_{b},\left\{1, A, N_{a}\right\}_{K_{b s}}, \\
S \rightarrow A: & N_{b},\left\{2, B, K_{a b}, N_{a}\right\}_{K_{a s}},\left\{3, A, B, K_{a b}, N_{b}\right\}_{K_{b s}}, \\
A \rightarrow B: & \left\{3, A, B, K_{a b}, N_{b}\right\}_{K_{b s}},\left\{4, N_{b}\right\}_{K_{a b}} .
\end{array}
$$


Otway-Rees. The Otway-Rees protocol can be informally described as follows:

$$
\begin{array}{ll}
A \rightarrow B: & M, A, B,\left\{N_{a}, M, A, B\right\} K_{a s}, \\
B \rightarrow S: & M, A, B,\left\{N_{a}, M, A, B\right\} K_{a s},\left\{N_{b}, M, A, B\right\} K_{b s}, \\
S \rightarrow B: & M,\left\{N_{a}, K_{a b}\right\} K_{a s},\left\{N_{b}, K_{a b}\right\} K_{b s}, \\
B \rightarrow A: & M,\left\{N_{a}, K_{a b}\right\} K_{a s} .
\end{array}
$$

This protocol is type-compliant, without requiring any additional tagging, using the same structure-preserving typing system as before. As for the Yahalom-Lowe protocol, type-compliance relies here on the direct encoding of tuples, and would not hold with nested pairs. Actually, we can also consider a different typing system that consists of typing the variables used to model ciphertext forwarding using a constant. This does not correspond to the expected type in a normal execution, but we can show that the protocol is type-compliant. This yields an interesting property w.r.t. attack search. Namely, we deduce that it is useless to instantiate these variables with complex terms when looking for an attack.

Denning Sacco with signature. This protocol, presented in Reference [13], can be seen as a simplified version of the Denning Sacco protocol. It can be can be informally described as follows:

$$
\begin{array}{ll}
A \rightarrow B: & A, B,\{\operatorname{sign}(\langle A, B, k\rangle, s k A)\}_{\mathrm{pub}(B)}, \\
B \rightarrow A: & \{m\}_{k} .
\end{array}
$$

This protocol is type-compliant, without requiring any additional tagging, using the same structure-preserving typing system as before.

\subsection{E-passport Protocols}

We also consider two authentication protocols used in the e-passport application; namely, the passive authentication (PA) protocol and the active authentication (AA) protocol. These protocols enable a reader (agent $R$ in the following informal specifications) to authenticate a passport (agent $P$ ). The two agents already possess sessions keys, thanks to a prior execution of a keyexchange protocol. For this reason, the security property we want to verify here is a variant of unlinkability: We want to model the inability for an attacker to distinguish between a scenario involving Passport 1 and two sessions of Passport 2, and a scenario involving two copies of Passport 1 and only one session of Passport 2. This models the inability for the attacker to link two sessions of a same passport together. We then investigate the type-compliance of the resulting configurations and summarize the results in Figure 3. Similarly to the key-exchange protocols, we consider a version of the protocol where each session has been duplicated, to ensure all possible unifications are observed.

Passive authentication. This protocol can be informally described as follows:

$$
\begin{array}{ll}
R \rightarrow P: & \{\text { read }\}_{k s e n c}, \operatorname{mac}\left(\{\text { read }\}_{k s e n c}, k m a c\right), \\
P \rightarrow R: & \{\text { data }\}_{k s e n c}, \operatorname{mac}\left(\{\text { data }\}_{k s e n c}, k m a c\right),
\end{array}
$$

where $\operatorname{data}_{P}=\left\langle d g_{P}, \operatorname{sign}\left(\operatorname{hash}\left(d g_{P}\right), s k D S\right)\right.$, hash $\left.\left(d g_{P}\right)\right\rangle$. This protocol is type-compliant, without requiring any additional tagging, using the same structure-preserving typing system as before. Type-compliance arises here from the fact that encrypted subterms in the formal specification of the protocol use a fixed key, the session key shared between the reader and the passport, preventing unification between messages other than the honest execution of the protocol.

Active authentication. This protocol can be informally described as follows:

$$
\begin{array}{ll}
R \rightarrow P: & \{\text { init }, r\}_{k s e n c}, \operatorname{mac}\left(\{\text { init }, r\}_{k s e n c}, k m a c\right), \\
P \rightarrow R: & \{\operatorname{sign}(\langle n, r\rangle, s k P)\}_{k s e n c}, \operatorname{mac}\left(\{\operatorname{sign}(\langle n, r\rangle, s k P)\}_{k s e n c}, k m a c\right) .
\end{array}
$$


This protocol is type-compliant, without requiring any additional tagging, using the same structure-preserving typing system as before, and following the same argument as for the PA protocol.

Unfortunately, the BAC protocol is out of the scope of our result, since our syntax does not allow one to model else branches.

\subsection{E-voting Protocols}

Helios [3] is an e-voting protocol that has been used in several real-life elections. We consider here a simplified version of this protocol (e.g., the authentication mechanism is replaced by a digital signature) whose informal description is given below:

$$
\begin{array}{ll}
V_{i} \rightarrow S: & \operatorname{sign}\left(\operatorname{raenc}\left(v_{i}, \operatorname{pub}(s k s), r_{i}\right), s k i\right) \\
S \rightarrow V_{1}, \ldots, V_{n}: & v_{i_{1}}, \ldots, v_{i_{n}} .
\end{array}
$$

First, the voter $V_{i}$ casts her vote $v_{i} \in\{$ yes, no $\}$. This is achieved by sending to the server a randomized encryption of $v_{i}$ with the public key pub $(s k s)$ of the server $S$. Then, after receiving the ballot, the server outputs the tally. The server simply sends the valid votes received during the voting phase in some random order.

As explained in Section 2.4, vote privacy can be modeled through the following equivalence:

$$
\begin{array}{r}
\left(\left\{P_{S}, P_{V}\left(c_{a}, s k a, \text { yes, } r_{a}\right), P_{V}\left(c_{b}, s k b, \text { no, } r_{b}\right)\right\} ; \phi_{0} ; \emptyset ; 0\right) \\
\approx_{t} \\
\left(\left\{P_{S}, P_{V}\left(c_{a}, s k a, \text { no, } r_{a}\right), P_{V}\left(c_{b}, s k b, \text { yes, } r_{b}\right)\right\} ; \phi_{0} ; \emptyset ; 0\right) .
\end{array}
$$

This corresponds to a simple scenario with two honest voters, where

- $P_{S}=\operatorname{in}\left(c, \operatorname{sign}\left(\operatorname{raenc}\left(x_{1}, \operatorname{pub}(s k s), z_{1}\right), s k a\right)\right)$. $\operatorname{in}\left(c, \operatorname{sign}\left(\operatorname{raenc}\left(x_{2}, \operatorname{pub}(s k s), z_{2}\right), s k b\right)\right)$. (out $\left.\left(c, x_{1}\right) \mid \operatorname{out}\left(c, x_{2}\right)\right)$

- $P_{V}(c, s k, v, r)=\operatorname{out}(c, \operatorname{sign}(\operatorname{raenc}(v, \operatorname{pub}(s k s), r), s k))$;

- $\phi_{0}=\left\{\mathrm{w}_{1} \triangleright \operatorname{pub}(s k s), \mathrm{w}_{2} \triangleright \operatorname{vk}(s k a), \mathrm{w}_{3} \triangleright \operatorname{vk}(s k b)\right\}$.

Type-compliance w.r.t. to a structure-preserving typing system can be obtained by simply giving the same initial type to $r_{a}, r_{b}, z_{1}$, and $z_{2}$, and we have to also give the same type to yes, no, $x_{1}$, and $x_{2}$. Thus, our typing result applies. In particular, if there is a witness of non-equivalence, then there is a well-typed witness of non-equivalence. Note that our protocol is not determinate due to the two outputs that occur in parallel on channel $c$. This means that our typing result holds for our notion of equivalence $\approx_{t}$ only (with static inclusion), we cannot conclude for trace equivalence with static equivalence.

We may want to consider a richer scenario involving a dishonest voter $s k c$. The modelling will be similar. We need to modify the server role to allow three ballots to be received. The dishonest voter does not need to be modeled. It is sufficient to add $s k c$ to the knowledge of the attacker (i.e., in $\phi_{0}$ ). Type-compliance holds with a similar typing system. Actually, this simplified version of the Helios protocol does not guarantee vote privacy (hence, the corresponding equivalence does not hold). This is due to the fact that we do not model that the server is supposed to discard duplicated ciphertexts. The dishonest voter with key $s k c$ can intercept the ciphertext of voter $A$, sign it, and send it to the server. The result of the election will be different on both sides of the equivalence, and this is observable by the attacker. The corresponds to the replay attack described in Reference [30].

To model a server performing such a weeding phase (where duplicated ballots are discarded), a non-trivial else branch is needed. This is out of the scope of our result. 


\section{CONCLUSION}

We have established a simplification result for both reachability and equivalence properties: If there is an attack, then there is a well-typed attack, which reduces the search space. Our result holds for a large class of cryptographic primitives, which encompasses asymmetric encryption, signatures, and hashes, and for a flexible notion of typing system, provided the underlying protocol is type-compliant: Any two unifiable encrypted subterms have the same type. Note that instead of considering the usual notion of trace equivalence, we study a weaker equivalence, which appears to coincide with the original one for determinate processes. As future work, we plan to explore how to lift our simplification result to standard trace equivalence. The difficulty is that it requires to transfer equalities both ways (from $P$ to $Q$ and conversely) for each trace inclusion. In our proof, those equalities are preserved through type compliance on the left side. Thus, to handle standard trace equivalence, we would first need to relate the typing systems of both protocols under study.

Another natural next step would be to extend results that rely on the previous small attack result [23], limited to symmetric encryption. For example, we could probably establish a novel decidability result for protocols with nonces, for an unbounded number of sessions, for a large class of cryptographic primitives, generalizing the approach of Reference [24]. Similarly, we plan to extend the tool SAT-Equiv [28], which (efficiently) decides trace equivalence for a bounded number of sessions. SAT-Equiv is currently limited to symmetric encryption, as it relies on Reference [23]. The extension to asymmetric encryption, signatures, and hashes will require further (provably correct) optimizations to preserve the efficiency of the tool.

Finally, typing results can potentially be used for composition results. Given two secure protocols $P$ and $Q$, they can be safely composed if, intuitively, the execution of $P$ does not interfere with the execution of $Q$ (and conversely). Such composition results have been established for trace properties [4, 25, 29] as well as equivalence properties [5]. We could probably use our typing result to establish composition for equivalence properties and a larger class of primitives and protocols, assuming that protocols have disjoint types that can be typically enforced by tagging.

\section{APPENDICES}

\section{A PROOFS OF SECTION 4}

\section{A.1 Some Preliminaries}

Lemma 4.3. Let $\phi$ be a $\Sigma_{0}$-frame, $R$ a $\Sigma_{0}$-recipe such that $R \phi \downarrow$ is a $\Sigma_{0}$-message, and $R^{\prime}$ be such that $R \rightarrow R^{\prime}$. We have that $R^{\prime}$ is a $\Sigma_{0}$-recipe, and $R^{\prime} \phi \downarrow=R \phi \downarrow$.

Proof. Since $R \rightarrow R^{\prime}$, we know that there exists a position $p$ in $R$, a rewriting rule $\ell^{\prime} \rightarrow r$ associated to $\ell \rightarrow r \in \mathcal{R}$, and a substitution $\sigma$ such that $\left.R\right|_{p}=\ell^{\prime} \sigma$, and $R^{\prime}=R[r \sigma]_{p}$. Since $R \phi \downarrow$ is a $\Sigma_{0}$-message, we know that $\left(\ell^{\prime} \sigma\right) \phi \downarrow$ is a $\Sigma_{0}$-message. Let $t_{1}^{\prime}, \ldots, t_{k}^{\prime}$ and des $\in \Sigma_{\mathrm{d}}$ be such that $\ell^{\prime}=\operatorname{des}\left(t_{1}^{\prime}, \ldots, t_{n}^{\prime}\right)$. We have that $\left(\ell^{\prime} \sigma\right) \phi=\operatorname{des}\left(\left(t_{1}^{\prime} \sigma\right) \phi, \ldots,\left(t_{k}^{\prime} \sigma\right) \phi\right)$. As $\left(\ell^{\prime} \sigma\right) \phi \downarrow$ is a $\Sigma_{0^{-}}$ message, it means that $\ell \rightarrow r$ applies, as it is the only rule reducing des. Hence, we have that $\operatorname{des}\left(\left(t_{1}^{\prime} \sigma\right) \phi \downarrow, \ldots,\left(t_{k}^{\prime} \sigma\right) \phi \downarrow\right) \rightarrow(r \sigma) \phi \downarrow$. We deduce that $\left(\ell^{\prime} \sigma\right) \phi \downarrow=(r \sigma) \phi \downarrow$, and therefore, we have that $R \phi \downarrow=R^{\prime} \phi \downarrow$.

Lemma 4.5. Let $\theta$ be a substitution with $\operatorname{dom}(\theta) \subseteq \Sigma_{\text {fresh }}$ and whose image contains $\Sigma_{0}^{-}$-recipes. Let $R$ be a $\Sigma_{0}^{+}$-recipe in normal form w.r.t. $\rightarrow$ such that $(R \theta) \phi \downarrow$ is a $\Sigma_{0}^{+}$-message for some $\Sigma_{0}^{-}$-frame $\phi$. We have that $R^{\prime}$ is a simple $\Sigma_{0}^{+}$-recipe for any $R^{\prime} \in S t(R)$.

Proof. We prove this result by structural induction on $R$.

Base case: $R \in \mathcal{W} \cup \Sigma_{0}^{+}$. In both cases, the result holds, since $R$ is a simple $\Sigma_{0}^{+}$-recipe. 
Induction case: We have that $R=\mathrm{f}\left(R_{1}, \ldots, R_{k}\right)$ for some $\mathrm{f} \in \Sigma$, and we know that $R_{1}, \ldots, R_{k}$ are in normal form w.r.t. $\downarrow$.

- Case $\mathrm{f} \in \Sigma_{\mathrm{c}}$. We have that $(R \theta) \phi \downarrow=\mathrm{f}\left(\left(R_{1} \theta\right) \phi \downarrow, \ldots,\left(R_{k} \theta\right) \phi \downarrow\right)$ is a $\Sigma_{0}^{+}$-message for some $\Sigma_{0}^{-}$ frame $\phi$, and thus $\left(R_{i} \theta\right) \phi \downarrow$ is a $\Sigma_{0}^{+}$-message for $i \in\{1, \ldots, k\}$. Applying our induction hypothesis on $R_{i}(1 \leq i \leq k)$, we easily conclude.

- Case $\mathrm{f}=\operatorname{des} \in \Sigma_{\mathrm{d}}$. Let $\ell_{\mathrm{des}} \rightarrow r_{\mathrm{des}}$ be the rule in $\mathcal{R}$ such that $\operatorname{root}\left(\ell_{\mathrm{des}}\right)=$ des, and $\ell_{\mathrm{des}}^{\prime} \rightarrow r_{\mathrm{des}}$ its associated forced rewriting rule. If $r_{\text {des }} \in \mathcal{T}_{0}\left(\Sigma_{\mathrm{c}}, \emptyset\right)$, then we have that $R \rightarrow r_{\text {des }}$, which is impossible, as $R$ is in normal form w.r.t. $\rightarrow$. Thus, there is a unique position $p_{0}$ of $\ell_{\text {des }}$ such that $\left.\ell_{\text {des }}\right|_{p_{0}}=r_{\text {des }}$ and $p_{0}=1 . p_{0}^{\prime}$. We know that each $R_{i}$ is in normal form w.r.t. $\rightarrow$, and we have $\left(R_{i} \theta\right) \phi \downarrow$ is a $\Sigma_{0}^{+}$-message $(1 \leq i \leq k)$, as $(R \theta) \phi \downarrow$ is a $\Sigma_{0}^{+}$-message. Thus, our induction hypothesis applies, and we deduce that any subterm of $R_{i}$ is a simple $\Sigma_{0}^{+}$-recipe $(1 \leq i \leq k)$. We first assume that $R_{1}$ is a subterm $\Sigma_{0}^{+}$-recipe. Let $\psi$ be $\Sigma_{0}^{+}$-frame such that $R \psi \downarrow$ is a $\Sigma_{0}^{+}$message. We have that $R \psi \downarrow \in S t\left(R_{1} \psi \downarrow\right) \subseteq S t(\psi)$. Thus, $R$ is a subterm $\Sigma_{0}^{+}$-recipe, and thus a simple $\Sigma_{0}^{+}$-recipe.

Otherwise, we have that $R_{1}=C\left[R_{1}^{\prime}, \ldots, R_{k^{\prime}}^{\prime}\right]$ for some context built using symbols from $\Sigma_{\mathrm{c}} \uplus \Sigma_{0}^{+}$, and each $R_{j}^{\prime}\left(1 \leq j \leq k^{\prime}\right)$ is a subterm $\Sigma_{0}^{+}$-recipe such that $\operatorname{root}\left(R_{j}^{\prime}\right) \notin \Sigma_{\mathrm{c}}$. We have that $R=\operatorname{des}\left(C\left[R_{1}^{\prime}, \ldots, R_{k^{\prime}}^{\prime}\right], R_{2}, \ldots, R_{k}\right)$, and $p_{0}$ does not correspond to a position of the context $C$, since $R$ is in normal form w.r.t. $\rightarrow$. Let $p^{\prime}$ be the longest prefix of $p_{0}^{\prime}$ that corresponds to a position of $C$. We have that $\left.C\left[R_{1}^{\prime}, \ldots, R_{k^{\prime}}^{\prime}\right]\right|_{p^{\prime}}=R_{j}^{\prime}$ for some $j$. Let $\psi$ be $\Sigma_{0}^{+}$-frame such that $R \psi \downarrow$ is a $\Sigma_{0}^{+}$-message. We have that $R \psi \downarrow \in S t\left(R_{j}^{\prime} \psi \downarrow\right) \subseteq S t(\psi)$, as $R_{j}^{\prime}$ is a subterm $\Sigma_{0}^{+}$-recipe. Thus, $R$ is a subterm $\Sigma_{0}^{+}$-recipe, and thus a simple $\Sigma_{0}^{+}$-recipe.

This allows us to conclude.

\section{A.2 Our Measure}

Lemma A.1. Let $\phi_{S}$ be a $\Sigma_{0}^{+}$-frame, and $R$ be a $\Sigma_{0}^{+}$-recipe. We have that $\mu_{\phi_{S}}^{2}(R) \geq 0$.

Proof. Let $R=R_{0}\left[R_{1}, \ldots, R_{n}\right]$ where $R_{0}$ is the hat of $R$. Since $R_{0}$ only contains constructors, we have that $R \phi_{S} \downarrow=R_{0}\left[R_{1} \phi_{S} \downarrow, \ldots, R_{n} \phi_{S} \downarrow\right]$. Hence, we have that $\left|R \phi_{S} \downarrow\right| \geq\left|R_{0}\right|=$ hat $(R)$, which implies $\mu_{\phi_{S}}^{2}(R) \geq 0$.

Lemma A.2. Let $<$ be an ordering on $\sum_{\mathrm{fresh}}, \ell_{\mathrm{des}} \rightarrow r_{\mathrm{des}}$ be a rewriting rule from $\mathcal{R}$ (as defined in Section 2.1), and $\sigma$ be a substitution such that $\operatorname{img}(\sigma) \subseteq \mathcal{T}\left(\Sigma, \Sigma_{0}^{+} \uplus \mathcal{N}\right)$.

We have that Multi $\left(r_{\mathrm{des}} \sigma\right)<\operatorname{Multi}\left(\ell_{\mathrm{des}} \sigma\right)$. This result also holds when considering the forced rewriting associated to a rewriting rule in $\mathcal{R}$.

Proof. First, we consider the case where $r_{\text {des }} \in \mathcal{T}_{0}\left(\Sigma_{\mathrm{c}}, \emptyset\right)$. In such a case, we have

$$
\operatorname{Multi}\left(r_{\text {des }} \sigma\right)=\operatorname{Multi}\left(r_{\text {des }}\right)<\{\text { des }\}<\operatorname{Multi}\left(\ell_{\text {des }} \sigma\right) \text {. }
$$

Now, we consider the case where $r_{\mathrm{des}} \in \operatorname{St}\left(\ell_{\mathrm{des}}\right)$. We have that:

$$
\operatorname{Multi}\left(r_{\mathrm{des}} \sigma\right)<\{\text { des }\} \uplus \operatorname{Multi}\left(r_{\mathrm{des}} \sigma\right) \leq \operatorname{Multi}\left(\ell_{\mathrm{des}} \sigma\right) \text {. }
$$

Thus, in both cases, we have that $\operatorname{Multi}\left(r_{\mathrm{des}} \sigma\right)<\operatorname{Multi}\left(\ell_{\mathrm{des}} \sigma\right)$. The proof regarding the case of a forced rewriting rule can be done in similar way.

Lemma A.3. Let $<$ be an ordering on $\Sigma_{\text {fresh }}, f \in \Sigma$ of arity $k$, and $t_{1}, \ldots, t_{k} \in \mathcal{T}(\Sigma, D)$ for some set $D$ of data. We have that Multi $\left(f\left(t_{1}, \ldots, t_{k}\right) \downarrow\right) \leq \operatorname{Multi}\left(f\left(t_{1} \downarrow, \ldots, t_{k} \downarrow\right)\right)$, and similarly for $\downarrow$.

Proof. First, we consider the case where $\mathrm{f}\left(t_{1}, \ldots, t_{k}\right) \downarrow=\mathrm{f}\left(t_{1} \downarrow, \ldots, t_{k} \downarrow\right)$. In such a situation, the result trivially holds. Thus, we know that $\mathrm{f}=\operatorname{des} \in \Sigma_{\mathrm{d}}$, and $\operatorname{des}\left(t_{1}, \ldots, t_{k}\right) \downarrow \neq \operatorname{des}\left(t_{1} \downarrow, \ldots, t_{k} \downarrow\right)$. It 
means that there exists a substitution $\sigma$ such that:

$$
\operatorname{des}\left(t_{1} \downarrow, \ldots, t_{k} \downarrow\right)=\ell_{\text {des }} \sigma \text { and } \operatorname{des}\left(t_{1}, \ldots, t_{k}\right) \downarrow=r_{\text {des }} \sigma .
$$

Thanks to Lemma A.2, we know that $\operatorname{Multi}\left(\operatorname{des}\left(t_{1}, \ldots, t_{k}\right) \downarrow<\operatorname{Multi}\left(\operatorname{des}\left(t_{1} \downarrow, \ldots, t_{k} \downarrow\right)\right)\right.$. This concludes the proof. A similar reasoning allows us to conclude regarding $\downarrow$.

Lemma 4.6. Let $<$ be an ordering on $\Sigma_{\text {fresh }}$, $\phi_{S}$ be a $\Sigma_{0}^{+}$-frame, $R_{1}, R_{2}$ be two $\Sigma_{0}^{+}$-recipes such that $R_{2} \phi_{S} \downarrow$ is not a $\Sigma_{0}^{+}$-message, and $\mu_{\phi_{S}}^{1}\left(R_{1}\right)<\mu_{\phi_{S}}^{1}\left(R_{2}\right)$. Let $R_{0}$ be a $\Sigma_{0}^{+}$-recipe, and p a position in $R_{0}$. We have that:

$$
\mu_{\phi_{S}}^{1}\left(R_{0}\left[R_{1}\right]_{p}\right)<\mu_{\phi_{S}}^{1}\left(R_{0}\left[R_{2}\right]_{p}\right) .
$$

Proof. We first establish the following claim by induction on the length of $p$ ( $\square$ is not a message):

$$
\operatorname{Multi}\left(R_{0}\left[R_{1}\right]_{p} \phi_{S} \downarrow\right) \leq\left(\operatorname{Multi}\left(R_{0} \phi_{S}[\square]_{p} \downarrow\right) \backslash\{\square\}\right) \uplus \operatorname{Multi}\left(R_{1} \phi_{S} \downarrow\right) .
$$

Base case: $p=\epsilon$. We have Multi $\left(R_{0} \phi_{S}[\square]_{p} \downarrow\right)=\{\square\}$. So,

$$
\operatorname{Multi}\left(R_{0}\left[R_{1}\right]_{p} \phi_{S} \downarrow\right)=\operatorname{Multi}\left(R_{1} \phi_{S} \downarrow\right)=\left(\operatorname{Multi}\left(R_{0} \phi_{S}[\square]_{p} \downarrow\right) \backslash\{\square\}\right) \uplus \operatorname{Multi}\left(R_{1} \phi_{S} \downarrow\right) .
$$

Inductive case: $p=j \cdot p^{\prime}$, and $R_{0}=\mathrm{f}\left(R_{1}^{\prime}, \ldots, R_{k}^{\prime}\right)$ for some $\Sigma_{0}^{+}$-recipes $R_{1}^{\prime}, \ldots, R_{k}^{\prime}$. Thanks to Lemma A.3, we obtain Multi $\left(R_{0}\left[R_{1}\right]_{p} \phi_{S} \downarrow\right) \leq \operatorname{Multi}\left(f\left(R_{1}^{\prime} \phi_{S} \downarrow, \ldots, R_{j}^{\prime}\left[R_{1}\right]_{p^{\prime}} \phi_{S} \downarrow, \ldots, R_{k}^{\prime} \phi_{S} \downarrow\right)\right)$, and thus

$$
\operatorname{Multi}\left(R_{0}\left[R_{1}\right]_{p} \phi_{S} \downarrow\right) \leq\{\mathrm{f}\} \uplus\left(\uplus_{i \neq j} \operatorname{Multi}\left(R_{i}^{\prime} \phi_{S} \downarrow\right)\right) \uplus \operatorname{Multi}\left(R_{j}^{\prime}\left[R_{1}\right]_{p^{\prime}} \phi_{S} \downarrow\right) .
$$

By induction hypothesis, we have: $\operatorname{Multi}\left(R_{j}^{\prime}\left[R_{1} \phi_{S} \downarrow\right]_{p^{\prime}}\right) \leq\left(\operatorname{Multi}\left(R_{j}^{\prime} \phi_{S}[\square]_{p^{\prime}} \downarrow\right) \backslash\{\square\}\right) \uplus$ $\operatorname{Multi}\left(R_{1} \phi_{S} \downarrow\right)$. Therefore, we have that:

$$
\operatorname{Multi}\left(R_{0}\left[R_{1}\right]_{p} \phi_{S} \downarrow\right) \leq\{\mathrm{f}\} \uplus\left(\uplus_{i \neq j} \operatorname{Multi}\left(R_{i}^{\prime} \phi_{S} \downarrow\right)\right) \uplus\left(\operatorname{Multi}\left(R_{j}^{\prime} \phi_{S}[\square]_{p^{\prime}} \downarrow\right) \backslash\{\square\}\right) \uplus \operatorname{Multi}\left(R_{1} \phi_{S} \downarrow\right) .
$$

But $R_{j}^{\prime} \phi_{S}[\square]_{p^{\prime}} \downarrow$ is not a message, and thus no reduction can occur above $\square$. Thus:

$$
\operatorname{Multi}\left(R_{0} \phi_{S}[\square]_{p} \downarrow\right)=\{\mathrm{f}\} \uplus\left(\uplus_{i \neq j} \operatorname{Multi}\left(R_{i}^{\prime} \phi_{S} \downarrow\right)\right) \uplus \operatorname{Multi}\left(R_{j}^{\prime}[\square]_{p^{\prime}} \phi_{S} \downarrow\right) .
$$

Hence, we deduce that:

$$
\operatorname{Multi}\left(R_{0} \phi_{S}[\square]_{p} \downarrow\right) \backslash\{\square\}=\{\mathrm{f}\} \uplus\left(\uplus_{i \neq j} \operatorname{Multi}\left(R_{i}^{\prime} \phi_{S} \downarrow\right) \uplus\left(\operatorname{Multi}\left(R_{j}^{\prime}[\square]_{p^{\prime}} \phi_{S} \downarrow\right) \backslash\{\square\}\right)\right.
$$

We deduce:

$$
\operatorname{Multi}\left(R_{0}\left[R_{1}\right]_{p} \phi_{S} \downarrow\right) \leq\left(\operatorname{Multi}\left(R_{0}[\square]_{p} \phi_{S} \downarrow\right) \backslash\{\square\}\right) \uplus \operatorname{Multi}\left(R_{1} \phi_{S} \downarrow\right) .
$$

This proves the claim.

Now, as $R_{2} \phi_{S} \downarrow$ is not a message, $R_{0}\left[R_{2}\right]_{p} \phi_{S} \downarrow=\left(\operatorname{Multi}\left(R_{0} \phi_{S}[\square]_{p} \downarrow\right) \backslash\{\square\}\right) \uplus \operatorname{Multi}\left(R_{2} \phi_{S} \downarrow\right)$. Since $\mu_{\phi_{S}}^{1}\left(R_{1}\right)<\mu_{\phi_{S}}^{1}\left(R_{2}\right)$, relying on our claim, we easily deduce that $\mu_{\phi_{S}}^{1}\left(R_{0}\left[R_{1}\right]_{p}\right)<\mu_{\phi_{S}}^{1}\left(R_{0}\left[R_{2}\right]_{p}\right)$.

Lemma 4.7. Let $<$ be an ordering on $\Sigma_{\text {fresh }}$, $\phi_{S}$ be a $\Sigma_{0}^{+}$-frame, and $R, R^{\prime}$ be two $\Sigma_{0}^{+}$-recipes such that $R \rightarrow R^{\prime}$. We have that $\mu_{\phi_{S}}\left(R^{\prime}\right)<\mu_{\phi_{S}}(R)$.

Proof. We first consider the case where $R \phi_{S} \downarrow=R^{\prime} \phi_{S} \downarrow$. Hence, we have that $\mu_{\phi_{S}}^{1}(R)=\mu_{\phi_{S}}^{1}\left(R^{\prime}\right)$, and we have also that $\mu^{3}(R)<\mu^{3}\left(R^{\prime}\right)$. Since $\left|R \phi_{S} \downarrow\right|=\left|R^{\prime} \phi_{S} \downarrow\right|$, to conclude, it only remains to establish that $\mid$ hat $\left(R^{\prime}\right)|\geq|$ hat $(R) \mid$. We have that $R=R_{0}\left[R_{1}, \ldots, R_{n}\right]$ with $R_{0}=$ hat $(R)$. Since $R \rightarrow R^{\prime}$, we know that $R^{\prime}=R_{0}\left[R_{1}, \ldots, R_{i}^{\prime}, \ldots, R_{n}\right]$ with $R_{i} \rightarrow R_{i}^{\prime}$, and thus $\mid$ hat $\left(R^{\prime}\right)|\geq| R_{0} \mid$.

Now, we consider the case where $R \phi_{S} \downarrow \neq R^{\prime} \phi_{S} \downarrow$. Let $\ell_{\text {des }}^{\prime} \rightarrow r_{\text {des }}$ be the rule applied to rewrite $R$ in $R^{\prime}$. We have that $R=R\left[\ell_{\text {des }}^{\prime} \delta\right]_{p}$ and $R^{\prime}=R\left[r_{\text {des }} \delta\right]_{p}$ for some position $p$, and some substitution $\delta$. Therefore, we have that $R \phi_{S} \downarrow=\left(R \phi_{S}\right)\left[\left(\ell_{\text {des }}^{\prime} \delta\right) \phi_{S} \downarrow\right]_{p} \downarrow$ and $R^{\prime} \phi_{S} \downarrow=\left(R \phi_{S}\right)\left[\left(r_{\mathrm{des}} \delta\right) \phi_{S} \downarrow\right]_{p} \downarrow$. By 
Lemma A.2, and as $\left(\left[\ell_{\mathrm{des}}^{\prime} \delta\right] \phi_{S} \downarrow\right)$ does not reduce (otherwise, we would have that $\left.R \phi_{S} \downarrow=R^{\prime} \phi_{S} \downarrow\right)$ ), we have that $\operatorname{Multi}\left(\left(\ell_{\text {des }}^{\prime} \delta\right) \phi_{S} \downarrow\right)>\operatorname{Multi}\left(\left[r_{\text {des }} \delta\right] \phi_{S} \downarrow\right)$. We deduce that $\mu_{\phi_{S}}^{1}\left(\ell_{\text {des }}^{\prime} \delta\right)>\mu_{\phi_{S}}^{1}\left(r_{\text {des }} \delta\right)$. Then Lemma 4.6 allows to conclude that $\mu_{\phi_{S}}^{1}\left(R^{\prime}\right)<\mu_{\phi_{S}}^{1}(R)$.

\section{A.3 Completeness}

LemmA A.4. Let $\phi_{S}$ be a $\Sigma_{0}^{+}$-frame together with $<$a total ordering on $\operatorname{dom}\left(\phi_{S}\right)$. Let $\theta$ be a substitu-

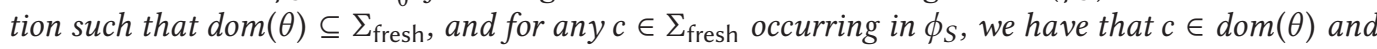
$c \theta \in \mathcal{T}\left(\Sigma, \Sigma_{0}^{-} \uplus \operatorname{dom}\left(\phi_{S}\right)\right)$. Moreover, we assume that in case $c \in \sum_{\text {fresh }}$ occurs in $\mathrm{w} \phi_{S}$, then $\mathrm{w}^{\prime} \prec \mathrm{w}$ for any $\mathrm{w}^{\prime} \in \operatorname{vars}(c \theta)$.

We have that any constant $c^{\prime} \in \sum_{\text {fresh }}$ that occurs in $(c \theta) \phi_{S} \downarrow$ is such that $\operatorname{rank}\left(c^{\prime}\right)<\operatorname{rank}(c)$ where $\left.\operatorname{rank}(c)=\max _{<}\{\mathrm{w} \mid \mathrm{w} \in \operatorname{vars}(c \theta)\}\right)$ when $\{\mathrm{w} \in \operatorname{vars}(c \theta)\} \neq \emptyset$, and $\perp$ otherwise.

Proof. Let $\phi_{S}$ be a $\Sigma_{0}^{+}$-frame as defined in the lemma, and $c \in \operatorname{dom}(\theta)$. Let $c^{\prime} \in \sum_{\text {fresh }}$ be a constant that occurs in $(c \theta) \phi_{S} \downarrow$. In case $\operatorname{rank}(c)=\perp$, then it means that $\operatorname{vars}(c \theta)=\emptyset$, and thus no constant from $\Sigma_{\text {fresh }}$ occurs in $c \theta$. Therefore, we are done. Now, let $w_{i}=\operatorname{rank}(c)$, we have that $\operatorname{vars}(c \theta) \subseteq\left\{\mathrm{w} \mid \mathrm{w} \leq \mathrm{w}_{i}\right\}$, and by hypothesis on $\theta$, we have that $\operatorname{rank}\left(c^{\prime}\right)<\mathrm{w}_{i}$ for any constant $c^{\prime} \in \Sigma_{\text {fresh }}$ occurring in $(c \theta) \phi_{S}$, and thus, we have that $\operatorname{rank}\left(c^{\prime}\right) \prec \operatorname{rank}(c)$.

LeMma 4.8. Let $\phi_{S}$ be a $\Sigma_{0}^{+}$-frame together with $\prec$ a total ordering on dom $\left(\phi_{S}\right)$. Let $\theta$ be a substitution such that $\operatorname{dom}(\theta) \subseteq \Sigma_{\mathrm{fresh}}$, and for any $c \in \Sigma_{\text {fresh }}$ occurring in $\phi_{S}$ we have that $c \in \operatorname{dom}(\theta)$ and $c \theta \in \mathcal{T}\left(\Sigma, \Sigma_{0}^{-} \uplus \operatorname{dom}\left(\phi_{S}\right)\right)$. Moreover, we assume that in case $c \in \Sigma_{\text {fresh }}$ occurs in $\mathrm{w} \phi_{S}$, then $\mathrm{w}^{\prime} \prec \mathrm{w}$ for any $\mathrm{w}^{\prime} \in \operatorname{vars}(c \theta)$. We consider the substitution $\lambda$ whose domain is dom $(\theta)$, and such that:

$$
c \lambda=(c \theta)\left(\phi_{S} \lambda\right) \downarrow \text { for any } c \in \operatorname{dom}(\lambda) .
$$

The substitution $\lambda$ is well-defined. Moreover, if $(c \theta) \phi_{S} \downarrow$ is a $\Sigma_{0}^{+}$-message for each $c \in \operatorname{dom}(\theta)$, and $(c \theta) \phi_{S} \downarrow$ is an atomic $\Sigma_{0}^{+}$-message when $c \in \Sigma_{\text {fresh }}^{\text {atom }}$, then $c \lambda$ is a $\Sigma_{0}^{-}$-message for each $c \in \operatorname{dom}(\lambda)$, and $c \lambda$ is an atomic $\Sigma_{0}^{-}$-message when $c \in \Sigma_{\text {fresh }}^{\text {atom }}$.

We call $\lambda$ the first-order substitution associated to $\theta$ through $\phi_{S}$.

Proof. Let $\operatorname{dom}\left(\phi_{S}\right)=\left\{\mathrm{w}_{1}, \ldots, \mathrm{w}_{n}\right\}$ be such that $\mathrm{w}_{1}<\mathrm{w}_{2}<\cdots<\mathrm{w}_{n}$. We show this result by induction on $\operatorname{rank}(c)$ relying on the order $\prec$.

Base case: $c \in \operatorname{dom}(\theta)$ such that $\operatorname{rank}(c)=\perp$. In such a case, we have that $\operatorname{vars}(c \theta)=\emptyset$, and thus $c \lambda=(c \theta) \downarrow$ is well-defined. Moreover, assuming that $(c \theta) \phi_{S} \downarrow=(c \theta) \downarrow$ is $\Sigma_{0}^{+}$-message, then we know that it is actually a $\Sigma_{0}^{-}$-message, and thus $c \lambda$ is a $\Sigma_{0}^{-}$-message that is atomic in case $(c \theta) \phi_{S} \downarrow$ is atomic.

Inductive step: $c \in \operatorname{dom}(\theta)$ such that $\operatorname{rank}(c)=\mathrm{w}_{i}$. Let $\lambda_{i}$ be the substitution that coincides with $\lambda$ on its domain $\operatorname{dom}\left(\lambda_{i}\right)=\left\{c^{\prime} \mid c^{\prime} \in \operatorname{dom}(\lambda)\right.$ and $\left.\operatorname{rank}\left(c^{\prime}\right)<\mathrm{w}_{i}\right\}$. We have that $\lambda_{i}$ is well-defined, and actually we have that $w_{j} \phi_{S} \lambda_{i}=w_{j} \phi_{S} \lambda$ for any $\mathrm{w}_{j} \leq \mathrm{w}_{i}$. Since $\operatorname{rank}(c)=\mathrm{w}_{i}$, we know that $\operatorname{vars}(c \theta) \subseteq\left\{\mathrm{w}_{1}, \ldots, \mathrm{w}_{i}\right\}$, and thus $c \lambda=(c \theta)\left(\phi_{S} \lambda\right) \downarrow=(c \theta)\left(\phi_{S} \lambda_{i}\right) \downarrow$ is well-defined. Moreover, assuming that $(c \theta) \phi_{S} \downarrow$ is a $\Sigma_{0}^{+}$-message, then we know that any constant $c^{\prime} \in \Sigma_{\text {fresh }}$ occurring in $(c \theta) \phi_{S} \downarrow$ is such that $\operatorname{rank}\left(c^{\prime}\right) \prec \mathrm{w}_{i}$ (thanks to Lemma A.4), and thus $c^{\prime} \lambda$ is a $\Sigma_{0}^{-}$-message, and an atomic one when $c \in \Sigma_{\text {fresh }}^{\text {atom }}$. Thus, we have that $(c \theta) \phi_{S} \downarrow \lambda$ is a $\Sigma_{0}^{-}$-message and an atomic one in case $c \in \sum_{\text {atom }}^{\text {fresh }}$. Actually, we have that $(c \theta) \phi_{S} \downarrow \lambda=(c \theta)\left(\phi_{S} \lambda\right) \downarrow$, which allows us to conclude.

LEMMA 4.9. Let $\phi_{S}$ be a $\Sigma_{0}^{+}$-frame together with $\prec$ a total ordering on dom $\left(\phi_{S}\right)$. Let $\theta$ be a substitution such that $\operatorname{dom}(\theta) \subseteq \Sigma_{\text {fresh }}$, and for any $c \in \Sigma_{\text {fresh }}$ occurring in $\phi_{S}$, we have that $c \in \operatorname{dom}(\theta)$ and $c \theta \in \mathcal{T}\left(\Sigma, \Sigma_{0}^{-} \uplus \operatorname{dom}\left(\phi_{S}\right)\right)$. Moreover, we assume that in case $c \in \sum_{\text {fresh }}$ occurs in $\mathrm{w} \phi_{S}$, then $\mathrm{w}^{\prime}<\mathrm{w}$ for any $\mathrm{w}^{\prime} \in \operatorname{vars}(c \theta)$. Let $\lambda$ be the first-order substitution associated to $\theta$ through $\phi_{S}$. 
Assume that for any $c \in \operatorname{dom}(\lambda)$, we have that $c \lambda$ is a $\Sigma_{0}^{-}$-message. Moreover, $c \lambda$ is an atomic $\Sigma_{0}^{-}$ message when $c \in \Sigma_{\text {fresh }}^{\text {atom }}$. Let $R \in \mathcal{T}\left(\Sigma, \Sigma_{0}^{-} \uplus \operatorname{dom}(\theta) \uplus \operatorname{dom}\left(\phi_{S}\right)\right)$ such that $R \phi_{S} \downarrow$ is a $\Sigma_{0}^{+}$-message. We have that $(R \theta)\left(\phi_{S} \lambda\right) \downarrow=\left(R \phi_{S} \downarrow\right) \lambda$.

Proof. We prove this result by structural induction on $R$.

Base case: $R \in \Sigma_{0}^{-} \uplus \operatorname{dom}(\theta) \uplus \operatorname{dom}\left(\phi_{S}\right)$. In case $R=c_{0} \in \Sigma_{0}^{-}$, then we have that $(c \theta)\left(\phi_{S} \lambda\right) \downarrow=$ $\left.c_{0}=\left(c \phi_{S} \downarrow\right) \lambda\right)$. In case $R=c \in \operatorname{dom}(\theta)$, then we have that $(c \theta)\left(\phi_{S} \lambda\right) \downarrow=c \lambda=\left(c \phi_{S} \downarrow\right) \lambda$, thanks to Lemma 4.8. In case $R=\mathrm{w} \in \operatorname{dom}\left(\phi_{S}\right)$, then we have that $(c \theta)\left(\phi_{S} \lambda\right) \downarrow=\mathrm{w} \phi_{S} \lambda \downarrow=\left(\mathrm{w} \phi_{S} \downarrow\right) \lambda$

Inductive step: $R \mathrm{f}\left(R_{1}, \ldots, R_{k}\right)$. In case $\mathrm{f} \in \Sigma_{\mathrm{c}}$, then we have that:

$$
(R \theta)\left(\phi_{S} \lambda\right) \downarrow=\mathrm{f}\left(\left(R_{1} \theta\right)\left(\phi_{S} \lambda\right) \downarrow, \ldots\left(R_{k} \theta\right)\left(\phi_{S} \lambda\right) \downarrow\right)=\mathrm{f}\left(R_{1} \phi_{S} \downarrow \lambda, \ldots R_{k} \phi_{S} \downarrow \lambda\right)=R \phi_{S} \downarrow \lambda .
$$

In case $\mathrm{f} \in \Sigma_{\mathrm{d}}$, then for each $i \in\{i, \ldots, k\}$, by hypothesis, we have that $R_{i} \phi_{S} \downarrow$ is a $\Sigma_{0}^{+}$-message. Thus, relying on our induction hypothesis, we have that:

$$
(R \theta)\left(\phi_{S} \lambda\right) \downarrow=\mathrm{f}\left(\left(R_{1} \theta\right)\left(\phi_{S} \lambda\right) \downarrow, \ldots,\left(R_{k} \theta\right)\left(\phi_{S} \lambda\right) \downarrow\right) \downarrow=\mathrm{f}\left(\left(R_{1} \phi_{S}\right) \downarrow \lambda, \ldots,\left(R_{k} \phi_{S}\right) \downarrow \lambda\right) \downarrow .
$$

However, we have that $R \phi_{S} \downarrow \lambda=\mathrm{f}\left(R_{1} \phi_{S} \downarrow, \ldots, R_{k} \phi_{S} \downarrow\right) \downarrow \lambda=\mathrm{f}\left(R_{1} \phi_{S} \downarrow, \ldots, R_{k} \phi_{S} \downarrow\right) \lambda \downarrow$ as $c \lambda$ is a $\Sigma_{0}^{-}$message whenever $c \in \operatorname{dom}(\lambda)$ and $c \lambda$ is an atomic $\Sigma_{0}^{-}$-message when $c \in \Sigma_{\text {fresh }}^{\text {atom }}$ by Lemma 4.8. So $R \phi_{S} \downarrow \lambda=\mathrm{f}\left(R_{1} \phi_{S} \downarrow \lambda, \ldots, R_{k} \phi_{S} \downarrow \lambda\right) \downarrow=(R \theta)\left(\phi_{S} \lambda\right) \downarrow$.

LeMma 4.10. Let $\mathcal{K}_{0}=\left(\mathcal{P}_{0} ; \phi_{0} ; \emptyset ; 0\right)$ be an initial $\Sigma_{0}$-configuration and $\mathcal{K}=(\mathcal{P} ; \phi ; \sigma ; i)$ be a $\Sigma_{0}$ configuration such that $\mathcal{K}_{0} \stackrel{\operatorname{tr}}{\Longrightarrow} \mathcal{K}$ for some tr w.r.t. $\Sigma_{0}$.

(1) We have that $\operatorname{ESt}(\mathcal{K} \sigma) \subseteq E \operatorname{St}\left(\mathcal{K}_{0} \sigma\right)$.

(2) Moreover, in case $\sigma$ is an $m g u$ between pairs of terms occurring in $E S t\left(\mathcal{K}_{0}\right)$, then we have that $\operatorname{ESt}(\mathcal{K} \sigma) \subseteq \operatorname{ESt}\left(\mathcal{K}_{0}\right) \sigma$.

Proof. We prove $\operatorname{ESt}\left(\mathcal{K} \sigma^{\prime}\right) \subseteq E S t\left(\mathcal{K}_{0} \sigma^{\prime}\right)$ for any $\sigma^{\prime}$ that coincide on $\sigma$ on $\operatorname{dom}(\sigma)$ by induction on the execution $\mathcal{K}_{0} \stackrel{\operatorname{tr}}{\rightarrow} \mathcal{K}$.

Base case: tr is empty. In such a case, the result is obvious.

Inductive case: $\operatorname{tr}=\operatorname{tr}_{0} \cdot \alpha$. We have that $\mathcal{K}_{0} \stackrel{\operatorname{tr}_{0}}{\longrightarrow} \mathcal{K}_{1} \stackrel{\alpha}{\rightarrow} \mathcal{K}$ where $\mathcal{K}_{1}=\left(\mathcal{P}_{1} ; \phi_{1} ; \sigma_{1} ; i_{1}\right)$, and $E S t\left(\mathcal{K}_{1} \sigma_{1}^{\prime}\right) \subseteq E S t\left(\mathcal{K}_{0} \sigma_{1}^{\prime}\right)$ for any $\sigma_{1}^{\prime}$ that coincides with $\sigma_{1}$ on $\operatorname{dom}\left(\sigma_{1}\right)$. We distinguish three cases depending on $\alpha$ :

- In case $\alpha$ is either a $\tau$ action or a phase $i$ action, then $\sigma=\sigma_{1}$ and $\operatorname{St}(\mathcal{K})=\operatorname{St}\left(\mathcal{K}_{1}\right)$. Therefore, let $\sigma^{\prime}$ be a substitution that coincides with $\sigma=\sigma_{1}$ on $\operatorname{dom}(\sigma)$, we have that $\operatorname{ESt}\left(\mathcal{K} \sigma^{\prime}\right)=$ $\operatorname{ESt}\left(\mathcal{K}_{1} \sigma^{\prime}\right) \subseteq \operatorname{ESt}\left(\mathcal{K}_{0} \sigma^{\prime}\right)$

- In case $\alpha$ is an input, then $\mathcal{P}_{1}=\operatorname{in}(c, u) . P \uplus \mathcal{P}_{1}^{\prime}$ and $\mathcal{P}=P \uplus \mathcal{P}_{1}^{\prime}$ so $\operatorname{St}(\mathcal{P}) \subseteq \operatorname{St}\left(\mathcal{P}_{1}\right)$. Moreover, $\sigma=\sigma_{1} \uplus \tau$ and $\operatorname{dom}(\tau)=\operatorname{vars}\left(u \sigma_{1}\right)$. Hence, we have that $\operatorname{ESt}(\tau) \subseteq \operatorname{ESt}\left(u \sigma_{1} \tau\right)=$ $\operatorname{ESt}(u \sigma)$. We also have $\phi=\phi_{1}$, and thus $\operatorname{ESt}(\phi)=E \operatorname{ESt}\left(\phi_{1}\right)$. Therefore, let $\sigma^{\prime}$ be a substitution that coincides with $\sigma$ on $\operatorname{dom}(\sigma)$. We have that $\operatorname{ESt}\left(\mathcal{K} \sigma^{\prime}\right) \subseteq E \operatorname{St}\left(\mathcal{K}_{1} \sigma^{\prime}\right)$. Note that $\sigma^{\prime}$ coincides with $\sigma_{1}$ on $\operatorname{dom}\left(\sigma_{1}\right)$; thus, thanks to our induction hypothesis, we know that $\operatorname{ESt}\left(\mathcal{K}_{1} \sigma^{\prime}\right) \subseteq \operatorname{ESt}\left(\mathcal{K}_{0} \sigma^{\prime}\right)$

- In case $\alpha$ is an output, then $\phi=\phi_{1} \uplus\left\{\mathrm{w} \triangleright u \sigma_{1}\right\}$ for some $u \in \operatorname{St}\left(\mathcal{K}_{1}\right)$ and $\sigma=\sigma_{1}$. Hence, we have that $\operatorname{ESt}(\phi) \subseteq E S t\left(\mathcal{K}_{1} \sigma_{1}\right)$. Let $\sigma^{\prime}$ be a substitution that coincides with $\sigma=\sigma_{1}$ on $\operatorname{dom}(\sigma)$, we have that $\operatorname{ESt}\left(\mathcal{K} \sigma^{\prime}\right) \subseteq \operatorname{ESt}\left(\mathcal{K}_{1} \sigma^{\prime}\right) \subseteq \operatorname{ESt}\left(\mathcal{K}_{0} \sigma^{\prime}\right)$.

This concludes the proof for the first item.

To prove item 2, we consider $\sigma$ the mgu between pairs of terms occurring in $\operatorname{ESt}\left(\mathcal{K}_{0}\right)$, and we show that $\operatorname{ESt}\left(\mathcal{K}_{0} \sigma\right) \subseteq E \operatorname{Et}\left(\mathcal{K}_{0}\right) \sigma$. Note that this inclusion together with item 1 allows us to 
conclude. Let $t \in \operatorname{ESt}\left(\mathcal{K}_{0} \sigma\right)$ be such that $t \notin E S t\left(\mathcal{K}_{0}\right) \sigma$. Therefore, we have that $t \in \operatorname{ESt}(\operatorname{img}(\sigma))$. Let $\bar{\sigma}=\sigma \delta$ where $\delta$ replaces any occurrence of $t$ in $\operatorname{img}(\sigma)$ by a fresh variable $x$. We have that:

- $u \bar{\sigma}=v \bar{\sigma}$ for any $u=v \in \Gamma$. Indeed, we know that $u \sigma=v \sigma$, and thus $(u \sigma) \delta=(v \sigma) \delta$. Since $t \notin E S t\left(\mathcal{K}_{0}\right) \sigma$, and $u, v \in E S t\left(\mathcal{K}_{0}\right)$, we deduce that $(u \sigma) \delta=u(\sigma \delta)=u \bar{\sigma}$ and similarly $(v \sigma) \delta=v(\sigma \delta)=v \bar{\sigma}$.

- $\bar{\sigma}$ is strictly more general than $\sigma$. Indeed, we have that $\sigma=\bar{\sigma} \tau$ considering $\tau=\{x \mapsto t\}$ and $t$ is an encrypted term, and thus not a variable.

This leads to a contradiction, since $\sigma=m g u(\Gamma)$ and concludes the proof.

Proposition 4.11. Let $\mathcal{K}_{P}=\left(\mathcal{P}_{0} ; \phi_{0} ; \emptyset ; i_{0}\right)$ be an initial $\Sigma_{0}^{-}$-configuration, and $(\operatorname{tr}, \phi) \in$ $\operatorname{trace}_{\Sigma_{0}^{-}}\left(\mathcal{K}_{P}\right)$ with underlying substitution $\sigma$. Then, there exists $\left(\operatorname{tr}_{S}, \phi_{S}\right) \in \operatorname{trace}_{\Sigma_{0}^{+}}\left(\mathcal{K}_{P}\right)$ with underlying substitution $\sigma_{S}$ with $\operatorname{dom}\left(\sigma_{S}\right)=\operatorname{dom}(\sigma)$ such that $\sigma_{S}=m g u(\Gamma) \rho$, as well as two substitutions $\lambda$ and $\theta$ such that:

- $\Gamma=\left\{(u, v) \mid u, v \in \operatorname{ESt}\left(\mathcal{K}_{P}\right)\right.$ such that $\left.u \sigma=v \sigma\right\}$.

- $\rho$ is a bijective renaming from variables in $\operatorname{dom}(\sigma) \backslash \operatorname{dom}(m g u(\Gamma))$ to constants in $\sum_{\text {fresh }}$ such that $x \rho \in \Sigma_{\text {fresh }}^{\text {atom }}$ if, and only if, $x \sigma$ is an atomic $\Sigma_{0}^{-}$-message.

- $\operatorname{dom}(\theta) \subseteq \sum_{\text {fresh }}$, for any $c \in \sum_{\text {fresh }}$ occurring in $\operatorname{tr}_{S}$, we have that $c \in \operatorname{dom}(\theta), c \theta \in \mathcal{T}\left(\Sigma, \Sigma_{0}^{-} \uplus\right.$ $\operatorname{dom}\left(\phi_{S}\right)$ ), and $\mathrm{w}^{\prime}<c$ for any $\mathrm{w}^{\prime} \in \operatorname{vars}(c \theta)$ (where $<$ is the ordering induced by $\operatorname{tr}_{S}$ ).

- for any $c \in \operatorname{dom}(\theta),(c \theta) \phi_{S} \downarrow$ is a $\Sigma_{0}^{+}$-message, and it is an atom when $c \in \sum_{\text {fresh }}^{\text {atom }}$.

- $\lambda$ is the first-order substitution associated to $\theta$ through $\phi_{S}$.

- $\phi=\phi_{S} \lambda, \sigma=\sigma_{S} \lambda$, and $\left(\operatorname{tr}_{S} \theta\right) \phi \downarrow=\operatorname{tr} \phi \downarrow$.

We say that $(\rho, \lambda, \theta)$ is a well-typed concretization of $\left(\operatorname{tr}_{S}, \phi_{S}\right)$ w.r.t. $(\operatorname{tr}, \phi)$ in the context $\mathcal{K}_{P}$ when $\rho, \lambda, \theta$ satisfy the six conditions listed above.

Proof. We know that $\mathcal{K}_{P} \stackrel{\text { tr }}{\Longrightarrow}(\mathcal{P} ; \phi ; \sigma ; i)$. Let $\Gamma=\left\{(u, v) \mid u, v \in E S t\left(\mathcal{K}_{P}\right)\right.$ such that $\left.u \sigma=v \sigma\right\}$, and $\rho$ be a bijective renaming from variables in $\operatorname{dom}(\sigma) \backslash \operatorname{dom}(m g u(\Gamma))$ to constants in $\sum_{\text {fresh }}$ such that $x \rho \in \sum_{\text {fresh }}^{\text {atom }}$ if and only if, $x \sigma$ is an atomic $\Sigma_{0}^{-}$-message. Let $\sigma_{S}$ be such that $\operatorname{dom}\left(\sigma_{S}\right)=\operatorname{dom}(\sigma)$ and $\sigma_{S}=m g u(\Gamma) \rho$. As $m g u(\Gamma)$ is more general than $\sigma$, we have $\sigma=m g u(\Gamma) \lambda_{0}$ for some $\lambda_{0}$. Let $\lambda=\rho^{-1} \lambda_{0}$. We have that $\sigma=\sigma_{S} \lambda$.

We show by induction on the length of a prefix $\mathcal{K}_{P} \stackrel{\operatorname{tr}^{+}}{\Longrightarrow}\left(\mathcal{P}^{+} ; \phi^{+} ; \sigma^{+} ; i^{+}\right)$of this execution trace that there exists $\left(\operatorname{tr}_{S}^{+}, \phi_{S}^{+}\right) \in \operatorname{trace}_{\Sigma_{0}^{+}}\left(\mathcal{K}_{P}\right)$ with underlying substitution $\sigma_{S}^{+}=\left.\sigma_{S}\right|_{\operatorname{dom}\left(\sigma^{+}\right)}$, as well as two substitutions $\theta^{+}$and $\lambda^{+}$such that:

- $\operatorname{dom}\left(\theta^{+}\right) \subseteq \sum_{\text {fresh }}$, for any $c \in \sum_{\text {fresh }}$ occurring in $\operatorname{tr}_{S}^{+}$, we have that $c \in \operatorname{dom}\left(\theta^{+}\right), c \theta^{+} \in$ $\mathcal{T}\left(\Sigma, \Sigma_{0}^{-} \uplus \operatorname{dom}\left(\phi_{S}^{+}\right)\right)$, and $\mathrm{w}^{\prime}<^{+} c$ for any $\mathrm{w}^{\prime} \in \operatorname{vars}\left(c \theta^{+}\right)$(where $<^{+}$is the ordering induced by $\left.\operatorname{tr}_{S}^{+}\right)$.

- for any $c \in \operatorname{dom}\left(\theta^{+}\right),\left(c \theta^{+}\right) \phi_{S}^{+} \downarrow$ is a $\Sigma_{0}^{+}$-message, and it is an atom when $c \in \sum_{\text {fresh }}^{\text {atom }}$.

- $\lambda^{+}$is the first-order substitution associated to $\theta^{+}$through $\phi_{S}^{+}$.

- $\phi^{+}=\phi_{S}^{+} \lambda^{+}, \sigma^{+}=\sigma_{S}^{+} \lambda^{+}$, and $\left(\operatorname{tr}_{S}^{+} \theta^{+}\right) \phi^{+} \downarrow=\operatorname{tr}^{+} \phi^{+} \downarrow$.

Base case: $\operatorname{tr}^{+}$is empty. In such a case, we have that $\operatorname{dom}\left(\sigma^{+}\right)=\emptyset$, and $\phi^{+}=\phi_{0}$. Let $\operatorname{tr}_{S}^{+}=\epsilon$, $\phi_{S}^{+}=\phi_{0}$, and $\sigma_{S}^{+}=\emptyset$. Choosing $\theta^{+}$and $\lambda^{+}$such that $\operatorname{dom}\left(\theta^{+}\right)=\operatorname{dom}\left(\lambda^{+}\right)=\emptyset$, the result trivially holds.

Inductive case: $\operatorname{tr}^{+}=\operatorname{tr}^{-} . \alpha$. In such a case, we have that:

$$
\mathcal{K}_{P}=\left(\mathcal{P}_{0} ; \phi_{0} ; \emptyset ; i_{0}\right) \stackrel{\operatorname{tr}^{-}}{\Longrightarrow}\left(\mathcal{P}^{-} ; \phi^{-} ; \sigma^{-} ; i^{-}\right) \stackrel{\alpha}{\Longrightarrow}\left(\mathcal{P}^{+} ; \phi^{+} ; \sigma^{+} ; i^{+}\right) .
$$


Thanks to our induction hypothesis applied on $\operatorname{tr}^{-}$, we know that there exists $\left(\operatorname{tr}_{S}^{-}, \phi_{S}^{-}\right) \in$ $\operatorname{trace}_{\Sigma_{0}^{+}}\left(\mathcal{K}_{P}\right)$ with underlying substitution $\sigma_{S}^{-}=\left.\sigma_{S}\right|_{d o m\left(\sigma^{-}\right)}$, as well as two substitutions $\theta^{-}$and $\lambda^{-}$such that:

- $\operatorname{dom}\left(\theta^{-}\right) \subseteq \sum_{\text {fresh }}$, for any $c \in \sum_{\text {fresh }}$ occurring in $\operatorname{tr}_{S}^{-}$, we have that $c \in \operatorname{dom}\left(\theta^{-}\right), c \theta^{-} \in$ $\mathcal{T}\left(\Sigma, \Sigma_{0}^{-} \uplus \operatorname{dom}\left(\phi_{S}^{-}\right)\right)$, and $\mathrm{w}^{\prime}<^{-} c$ for any $\mathrm{w}^{\prime} \in \operatorname{vars}\left(c \theta^{-}\right)$(where $<^{-}$is the ordering induced by $\left.\operatorname{tr}_{S}^{-}\right)$.

- for any $c \in \operatorname{dom}\left(\theta^{-}\right),\left(c \theta^{-}\right) \phi_{S}^{-} \downarrow$ is a $\Sigma_{0}^{+}$-message, and it is an atom when $c \in \Sigma_{\text {fresh }}^{\text {atom }}$.

- $\lambda^{-}$is the first-order substitution associated to $\theta^{-}$through $\phi_{S}^{-}$.

- $\phi^{-}=\phi_{S}^{-} \lambda^{-}, \sigma^{-}=\sigma_{S}^{-} \lambda^{-}$, and $\left(\operatorname{tr}_{S}^{-} \theta^{-}\right) \phi^{-} \downarrow=\operatorname{tr}^{-} \phi^{-} \downarrow$.

Let $c \in \Sigma_{\text {fresh }}$ and $\mathrm{w} \in \operatorname{dom}\left(\phi_{S}^{-}\right)$such that $c$ occurs in $\mathrm{w} \phi_{S}$. Then, as $c$ does not occur in the $\Sigma_{0}^{-}$configuration $\mathcal{K}_{P}, c$ must have been introduced in an input before the output of $\mathrm{w}$. So $c<^{-} \mathrm{w}$. Let $\mathrm{w}^{\prime} \in \operatorname{vars}\left(c \theta^{-}\right)$. Then $\mathrm{w}^{\prime}<^{-} c$ by definition of $<^{-}$. So $\mathrm{w}^{\prime}<^{-} \mathrm{w}$ and the order induced by $<^{-}$on $\operatorname{dom}\left(\phi_{S}^{-}\right)$satisfies the condition of Lemma 4.8 and Lemma 4.9. We distinguish three cases depending on $\alpha$.

Case where $\alpha=$ phase $j$ for some integer $j$. In such a case, we have that $\mathcal{P}^{+}=\mathcal{P}^{-}, \phi^{+}=\phi^{-}, \sigma^{+}=$ $\overline{\sigma^{-} \text {, and } i^{+}=j>i^{-} \text {. Let } \mathcal{P}_{S}^{+}=\mathcal{P}_{S}^{-}, \phi_{S}^{+}=\phi_{S}^{-}}, \sigma_{S}^{+}=\sigma_{S}^{-}$, and $i_{S}^{+}=j$. Since $i_{S}^{-}=i^{-}<j$, we have that:

$$
\left(\mathcal{P}_{S}^{-} ; \phi_{S}^{-} ; \sigma_{S}^{-} ; i_{S}^{-}\right) \stackrel{\text { phase } j}{\Longrightarrow}\left(\mathcal{P}_{S}^{+} ; \phi_{S}^{+} ; \sigma_{S}^{+} ; j\right)=\left(\mathcal{P}_{S}^{+} ; \phi_{S}^{+} ; \sigma_{S}^{+} ; i_{S}^{+}\right) \text {. }
$$

Considering $\theta^{+}=\theta^{-}, \lambda^{+}=\lambda^{-}$, and $<^{+}=<^{-}$, it is easy to show that all our requirements are satisfied. Case where $\alpha=\operatorname{out}\left(c, \mathrm{w}_{0}\right)$. In such a case, we have that $\mathcal{P}^{-}=\left\{\right.$out $\left.(c, u) . P_{c}\right\} \uplus Q$ for some $u, P_{c}$, $\overline{\text { and } Q, \mathcal{P}^{+}=\left\{P_{c}\right\} \uplus Q, \phi^{+}}=\phi^{-} \uplus\left\{\mathrm{w}_{0} \triangleright u \sigma^{-}\right\}, \sigma^{+}=\sigma^{-}$, and $i^{+}=i^{-}$. Let $\mathcal{P}_{S}^{+}=\left\{P_{c}\right\} \uplus Q, \phi_{S}^{+}=\phi_{S}^{-} \uplus$ $\left\{\mathrm{w}_{0} \triangleright u \sigma_{S}^{-}\right\}, \sigma_{S}^{+}=\sigma_{S}^{-}$, and $i_{S}^{+}=i_{S}^{-}$. We define $\theta^{+}=\theta^{-}$and $\lambda^{+}=\lambda^{-}$.

First, we need to show that $u \sigma_{S}^{-}$is a $\Sigma_{0}^{+}$-message. Thanks to our induction hypothesis, we know that $u \sigma^{-}=u \sigma_{S}^{-} \lambda^{-}$, and by hypothesis, we have that $u \sigma^{-}$is a $\Sigma_{0}^{-}$-message. Thus, to conclude, we only have to show that $c \lambda^{-} \in \Sigma_{0}^{-}$implies that $c \in \Sigma_{\text {fresh }}^{\text {atom }}$ for any $c \in \Sigma_{\text {fresh }}$ occurring in $u \sigma_{S}^{-}$. Let

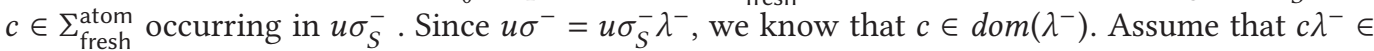
$\Sigma_{0}^{-}$and let $x \in \operatorname{dom}(\sigma) \backslash \operatorname{dom}(m g u(\Gamma))$ be the unique variable such that $\rho(x)=c$. We have that $x m g u(\Gamma)=x$; thus, we deduce that $c \lambda^{-}=((x m g u(\Gamma)) \rho) \lambda^{-}=x \sigma_{S}^{-} \lambda^{-}=x \sigma^{-}$. Hence, we have that $x \sigma \in \Sigma_{0}^{-}$, and thus $\rho(x)=c \in \Sigma_{\text {fresh }}^{\text {atom }}$ by definition of the renaming $\rho$.

Therefore, we have shown that $u \sigma_{S}^{-}$is a $\Sigma_{0}^{+}$-message, and thus we have that:

$$
\left(\mathcal{P}_{S}^{-} ; \phi_{S}^{-} ; \sigma_{S}^{-} ; i_{S}^{-}\right) \stackrel{\operatorname{out}\left(c, \mathrm{w}_{0}\right)}{\Longrightarrow}\left(\mathcal{P}_{S}^{+} ; \phi_{S}^{-} \uplus\left\{\mathrm{w} \triangleright u \sigma_{S}^{-}\right\} ; \sigma_{S}^{-} ; i_{S}^{-}\right)=\left(\mathcal{P}_{S}^{+} ; \phi_{S}^{+} ; \sigma_{S}^{+} ; i_{S}^{+}\right) .
$$

Considering $\theta^{+}=\theta^{-}, \lambda^{+}=\lambda^{-}$, and $<^{+}$the extension of $<^{-}$with $u<^{+} w_{0}$ for any $u \in \mathcal{W} \uplus \Sigma_{\text {fresh }}$ occurring in $\operatorname{tr}_{S}^{-}$or in $\operatorname{dom}\left(\phi_{0}\right)$, it is easy to show that all our requirements are satisfied.

Case where $\alpha=\operatorname{in}(c, R)$. We have that $\mathcal{P}^{-}=\left\{\operatorname{in}(c, u) . P_{c}\right\} \cup Q$ for some $u \in \mathcal{T}_{0}\left(\Sigma_{c}, \Sigma_{0}^{-} \uplus \mathcal{N}\right)$ and

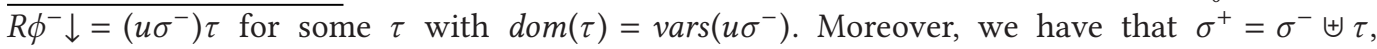
$\left(u \sigma^{-}\right) \tau=u \sigma^{+}, \phi^{+}=\phi^{-}$, and $\mathcal{P}^{+}=\left\{P_{c}\right\} \cup Q$. We consider $R_{S}$ minimal w.r.t. $\mu_{\phi_{S}^{-}}$such that $\left(R_{S} \theta^{-}\right) \phi^{-} \downarrow=\left(u \sigma^{-}\right) \tau=u \sigma^{+}$. Note that such an $R_{S}$ exists, since $R_{S}=R$ is actually a candidate (but not necessary a minimal one). We can assume w.l.o.g. that $R_{S}$ only uses constants from $\Sigma_{\text {fresh }}$ that have been introduced by $\operatorname{tr}_{S}^{-}$, and thus that are in $\operatorname{dom}\left(\theta^{-}\right)$.

Step 1: We prove that $R_{S} \phi_{S}^{-} \downarrow$ is a $\Sigma_{0}^{+}$-message. Assume that $R_{S} \phi_{S}^{-} \downarrow$ is not a $\Sigma_{0}^{+}$-message. We take the smallest subterm $R^{\prime}$ of $R_{S}$ such that $R^{\prime} \phi_{S}^{-} \downarrow$ is not a $\Sigma_{0}^{+}$-message. Let $p$ be such that $\left.R_{S}\right|_{p}=R^{\prime}$. As $R^{\prime} \phi_{S}^{-} \downarrow$ is not a $\Sigma_{0}^{+}$-message, we know that $R^{\prime} \notin \Sigma_{0}^{+} \uplus \operatorname{dom}\left(\phi_{S}^{-}\right)$. Thus, we have 
that $R^{\prime}=\mathrm{f}\left(R_{1}, \ldots, R_{k}\right)$ for some $\mathrm{f} \in \Sigma$. Moreover, by minimality of $R^{\prime}$, we know that $R_{i} \phi_{S}^{-} \downarrow$ is a $\Sigma_{0}^{+}$-message for $1 \leq i \leq k$. We now establish the following claim:

Claim. If $R_{i} \phi_{S}^{-} \downarrow \in \sum_{\text {fresh }}$ for some $i \in\{1, \ldots, k\}$, then $R_{S}$ is not minimal.

Proof. Assume $R_{i} \phi_{S}^{-} \downarrow=c \in \Sigma_{\text {fresh }}$ for some $i \in\{1, \ldots, k\}$. Note that this implies that $c$ occurs in $\phi_{S}^{-}$, and thus $c$ occurs in $\operatorname{tr}_{S}$, and therefore $c \in \operatorname{dom}\left(\theta^{-}\right)$. We consider $R_{i}^{\prime}=c \theta^{-}$. Thanks to Lemma 4.9, we have that $\left(R_{i} \theta^{-}\right) \phi^{-} \downarrow=R_{i} \phi_{S}^{-} \downarrow \lambda^{-}$. We have that $R_{i} \phi_{S}^{-} \downarrow \lambda^{-}=c \lambda^{-}=\left(c \theta^{-}\right)\left(\phi_{S}^{-} \lambda^{-}\right) \downarrow$, since $\lambda^{-}$is the first-order substitution associated to $\theta^{-}$through $\phi_{S}^{-}$. Thus, we have that $\left(R_{i} \theta^{-}\right) \phi^{-} \downarrow=$ $\left(c \theta^{-}\right) \phi^{-} \downarrow=\left(R_{i}^{\prime} \theta^{-}\right) \phi^{\prime} \downarrow$. Let $\overline{R_{S}}=R_{S}\left[\mathrm{f}\left(R_{1}, \ldots, R_{i}^{\prime}, \ldots, R_{k}\right)\right]_{p}$. We have that $\left(\overline{R_{S}} \theta^{-}\right) \phi^{-} \downarrow=\left(R_{S} \theta^{-}\right) \phi^{-} \downarrow$. We have also that $\mu_{\phi_{S}^{-}}^{1}\left(R_{i}^{\prime}\right)<\mu_{\phi_{S}^{-}}^{1}\left(R_{i}\right)$, since $R_{i}^{\prime} \phi_{S}^{-} \downarrow=\left(c \theta^{-}\right) \phi_{S}^{-} \downarrow$ is a $\Sigma_{0}^{+}$-message, and it is an atom when $c \in \Sigma_{\text {fresh }}^{\text {atom }}$, and $R_{i} \phi_{S}^{-} \downarrow=c$. Thus, thanks to Lemma 4.6, we deduce that $\mu_{\phi_{S}^{-}}^{1}\left(\overline{R_{S}}\right)<\mu_{\phi_{S}^{-}}^{1}\left(R_{S}\right)$. Thus, $R_{S}$ is not minimal, and this proves the claim.

We now distinguish two cases depending on whether $\mathrm{f} \in \Sigma_{\mathrm{c}}$ or $\mathrm{f} \in \Sigma_{\mathrm{d}}$.

Case where $\mathrm{f} \in \Sigma_{\mathrm{c}} . R^{\prime} \phi_{S}^{-} \downarrow$ is either not well-shaped or not well-sorted, as it is not a $\Sigma_{0}^{+}$-message. If it is not well-sorted, then for one of its subrecipes $R_{i}, R_{i} \phi_{S}^{-} \downarrow$ is not an atom (it is well-sorted by minimality of $R^{\prime}$ ) while it should be. In particular, $\left(R_{i} \theta^{-}\right) \phi^{-} \downarrow=R_{i} \phi_{S}^{-} \downarrow \lambda^{-}$is an atom. So $R_{i} \phi_{S}^{-} \downarrow$ must be in $\Sigma_{\text {fresh }}$, which will contradict the minimality of $R_{S}$, thanks to our claim. We deduce that $R^{\prime} \phi_{S}^{-} \downarrow$ is well-sorted.

Now, we assume that $R^{\prime} \phi_{S}^{-} \downarrow$ is not well-shaped, we consider the shape of $\mathrm{f}, \operatorname{sh}_{\mathrm{f}}=\mathrm{f}\left(s_{1}, \ldots, s_{k}\right)$ for some $s_{1}, \ldots, s_{k}$. As $R^{\prime} \phi_{S}^{-} \downarrow$ has a bad shape and is an $\mathrm{f}$-term, there must be a $j$ such that $R_{j} \phi_{S}^{-} \downarrow$ is not an instance of $s_{j}$. In particular, $s_{j}$ is not a variable and we have that $s_{j}=s h_{\mathrm{g}}$ for some function symbol g (thanks to the compatibility of the shapes). We know that $R_{j} \phi_{S}^{-} \downarrow$ is a $\Sigma_{0}^{+}$-message, and thus we have that $\left(R_{j} \theta^{-}\right) \phi^{-} \downarrow=\left(R_{j} \phi_{S}^{-} \downarrow\right) \lambda^{-}$(thanks to Lemma 4.9) is an instance of $s_{j}$, as $\left(R \theta^{-}\right) \phi^{-} \downarrow$ is a $\Sigma_{0}^{-}$-message. Relying on our claim, we know that $R_{j} \phi_{S}^{-} \downarrow \notin \Sigma_{\text {fresh }}$, thus $R_{j} \phi_{S}^{-} \downarrow$ is a g-term, and since we know that $R_{j} \phi_{S}^{-} \downarrow$ is a $\Sigma_{0}^{+}$-message, we know that it is an instance of $s_{j}$, yielding to a contradiction.

Case where $\mathrm{f}=\operatorname{des} \in \Sigma_{\mathrm{d}}$. In such a case, we have that $R^{\prime}=\operatorname{des}\left(R_{1}, \ldots, R_{k}\right)$. Let $\ell_{\text {des }}=\operatorname{des}\left(t_{1}\right.$, $\left.t_{2}, \ldots, t_{k}\right)$. We distinguish two subcases:

First, we assume that there is some $i \in\{1, \ldots, k\}$ such that $R_{i} \phi_{S}^{-} \downarrow$ does not unify with $t_{i}$. As $R_{i} \phi_{S}^{-} \downarrow$ is a $\Sigma_{0}^{+}$-message, it has a good shape. Thus, the only way to not unify with the linear term $t_{i}$ whereas $R_{i} \phi_{S}^{-} \downarrow \lambda^{-}=\left(R_{i} \theta^{-}\right) \phi^{-} \downarrow$ (thanks to Lemma 4.9) does, is when $R_{i} \phi_{S}^{-} \downarrow=c$ for some $c \in \sum_{\text {fresh. }}$. Relying on our claim, we obtain a contradiction.

Second, we assume that $R_{i} \phi_{S}^{-} \downarrow$ unifies with $t_{i}$ for each $i \in\{1, \ldots k\}$. In such a case, we know that $\ell_{\text {des }}=\operatorname{des}\left(t_{1}, t_{2}, \ldots, t_{k}\right)$ is a non-linear term and we denote $x$ the non-linear variable occurring in $\ell_{\text {des }}$. Let $I_{0}=\left\{1 \leq i \leq k \mid x\right.$ occurs in $\left.t_{i}\right\}$. We know that $1 \in I_{0}$. For any $i \in I_{0}$, we denote $p_{i}$ the position in $t_{i}$ such that $\left.t_{i}\right|_{p_{i}}=x$.

Since $R^{\prime} \phi_{S}^{-} \downarrow \lambda^{-}$is a $\Sigma_{0}^{-}$-message, we know that $t=\operatorname{des}\left(R_{1} \phi_{S}^{-} \downarrow \lambda^{-}, \ldots, R_{k} \phi_{S}^{-} \downarrow \lambda^{-}\right)$unifies with $\operatorname{des}\left(t_{1}, \ldots, t_{k}\right)$. Therefore, we know that there exists an atomic $\Sigma_{0}^{-}$-message $a$ such that $\left.t\right|_{i . p_{i}}=a$ for any $i \in I_{0}$. We know that $R^{\prime} \phi_{S}^{-} \downarrow$ is not a $\Sigma_{0}^{+}$-message whereas $R_{i} \phi_{S}^{-} \downarrow$ are $\Sigma_{0}^{+}$-message. Thus, we have that $t_{S}=\operatorname{des}\left(R_{1} \phi_{S}^{-} \downarrow, \ldots, R_{k} \phi_{S}^{-} \downarrow\right)$ does not unify with $\operatorname{des}\left(t_{1}, \ldots, t_{k}\right)$. We deduce that for any $i \in I_{0}$, we have that either $\left.t_{S}\right|_{i . p_{i}}=a$, or $\left.t_{S}\right|_{i . p_{i}}=c$ for some $c \in \sum_{\text {fresh }}$ such that $c \lambda^{-}=a$. We distinguish two cases depending on whether $\left.R_{1} \phi_{S}^{-} \downarrow\right|_{p_{1}}=c^{\prime}$ for some $c^{\prime} \in \Sigma_{\text {fresh }}$ or not:

First, we assume that $R_{1} \phi_{S}^{-} \downarrow \|_{p_{1}}=c^{\prime}$ for some $c^{\prime} \in \sum_{\text {fresh. }}$. Let $v_{\text {des }}$ be the substitution such that $x v_{\text {des }}=c^{\prime}$ and $y v_{\text {des }}=c_{\text {min }}$ for any other variable $y \in \operatorname{vars}\left(\ell_{\text {des }}\right)$. Let $\overline{R^{\prime}}=\operatorname{des}\left(R_{1}, t_{2} v_{\text {des }}, \ldots, t_{k} v_{\text {des }}\right)$. Actually, we have that $\overline{R^{\prime}} \phi_{S}^{-} \downarrow$ is a $\Sigma_{0}^{+}$-message, and thanks to Lemma 4.9, we know that $\left(\overline{R^{\prime}} \theta^{-}\right) \phi^{-} \downarrow=$ $\overline{R^{\prime}} \phi_{S}^{-} \downarrow \lambda^{-}$. Since $\overline{R^{\prime}} \phi_{S}^{-} \downarrow$ is a $\Sigma_{0}^{+}$-message, we have that $\mu_{\phi_{S}^{-}}^{1}\left(\overline{R^{\prime}}\right)<\{$ des $\}$, and thus $\mu_{\phi_{S}^{-}}^{1}\left(\overline{R^{\prime}}\right)<\mu_{\phi_{S}^{-}}^{1}\left(R^{\prime}\right)$. 
We also deduce that $\left(\overline{R^{\prime}} \theta^{-}\right) \phi^{-} \downarrow$ is a $\Sigma_{0}^{-}$-message, and we have that $\left(\overline{R^{\prime}} \theta^{-}\right) \phi^{-} \downarrow=\left(R^{\prime} \theta^{-}\right) \phi^{-} \downarrow$, since the $\Sigma_{0}^{+}$-recipes $\overline{R^{\prime}}$ and $R^{\prime}$ coincide on their first argument.

Second, we assume that $R_{1} \phi_{S}^{-} \downarrow_{p_{1}} \notin \sum_{\text {fresh }}$, i.e., $R_{1} \phi_{S}^{-} \downarrow_{p_{1}}=a$. We know that there exists $i_{0} \in I_{0}$ such that $\left.R_{i_{0}} \phi_{S}^{-} \downarrow\right|_{p_{i_{0}}}=c^{\prime}$ for some $c^{\prime} \in \sum_{\text {fresh }}$ (otherwise, $t_{S}$ will reduce), and we have that $c^{\prime} \lambda^{-}=$ $a$. Let $v_{\text {des }}$ be the substitution such that $x v_{\text {des }}=c^{\prime} \theta^{-}$and $y v_{\text {des }}=c_{\text {min }}$ for any other variable $y \in \operatorname{vars}\left(\ell_{\mathrm{des}}\right)$. Let $\overline{R^{\prime}}=\operatorname{des}\left(R_{1}, t_{2} v_{\mathrm{des}}, \ldots, t_{k} v_{\mathrm{des}}\right)$. As $\mu_{\phi_{S}^{-}}^{1}\left(c^{\prime} \theta^{-}\right)<\mu_{\phi_{S}^{-}}^{1}\left(c^{\prime}\right)$, we have that $\mu_{\phi_{S}^{-}}^{1}\left(R_{1}\right) \uplus$ $\mu_{\phi_{S}^{-}}^{1}\left(t_{2} v_{\text {des }}\right) \uplus \ldots \uplus \mu_{\phi_{S}^{-}}^{1}\left(t_{k} v_{\text {des }}\right)<\mu_{\phi_{S}^{-}}^{1}\left(R_{1}\right) \uplus \ldots \uplus \mu_{\phi_{S}^{-}}^{1}\left(R_{k}\right)$. Therefore, relying on Lemma A.3, we deduce that:

$$
\begin{aligned}
\mu_{\phi_{S}^{-}}^{1}\left(\overline{R^{\prime}}\right) & =\operatorname{Multi}\left(\overline{R^{\prime}} \phi_{S}^{-} \downarrow\right) \\
& \leq \operatorname{Multi}\left(\operatorname{des}\left(R_{1} \phi_{S}^{-} \downarrow, t_{2} v_{\text {des }} \phi_{S}^{-} \downarrow, \ldots, t_{k} v_{\text {des }} \phi_{S}^{-} \downarrow\right)\right) \\
& =\{\text { des }\} \uplus \mu_{\phi_{S}^{-}}^{1}\left(R_{1}\right) \uplus \mu_{\phi_{S}^{-}}^{1}\left(t_{2} v_{\text {des }}\right) \uplus \cdots \uplus \mu_{\phi_{S}^{-}}^{1}\left(t_{k} v_{\text {des }}\right) \\
& <\{\text { des }\} \uplus \mu_{\phi_{S}^{-}}^{1}\left(R_{1}\right) \uplus \cdots \uplus \mu_{\phi_{S}^{-}}^{1}\left(R_{k}\right) \\
& =\mu_{\phi_{S}^{-}}^{1}\left(R^{\prime}\right) .
\end{aligned}
$$

Since $R_{1} \phi_{S}^{-} \downarrow$ is a $\Sigma_{0}^{+}$-message, we have that $\operatorname{des}\left(\left(R_{1} \theta^{-}\right) \phi^{-} \downarrow,\left(\left(t_{2} v_{\text {des }}\right) \theta^{-}\right) \phi^{-} \downarrow, \ldots,\left(\left(t_{k} v_{\text {des }}\right) \theta^{-}\right) \phi^{-} \downarrow\right)=$ $\operatorname{des}\left(\left(R_{1} \phi_{S}^{-}\right) \downarrow \lambda^{-},\left(\left(t_{2} v_{\text {des }}\right) \theta^{-}\right) \phi^{-} \downarrow, \ldots,\left(\left(t_{k} v_{\text {des }}\right) \theta^{-}\right) \phi^{-} \downarrow\right)$. Such a term unifies with $\ell_{\text {des }}$, since we have that $\left.R_{1} \phi_{S}^{-} \downarrow \lambda^{-}\right|_{p_{1}}=\left.R_{1} \phi_{S}^{-} \downarrow\right|_{p_{1}} \lambda^{-}=a \lambda^{-}=a$, and for any $i \in I_{0}$, we have that:

$$
\left.\left(\left(t_{i} v_{\mathrm{des}}\right) \theta^{-}\right) \phi^{-} \downarrow\right|_{p_{i}}=\left.\left(t_{i} v_{\mathrm{des}}\right) \phi^{-} \downarrow\right|_{p_{i}}=\left(\left.\left(t_{i} v_{\mathrm{des}}\right)\right|_{p_{i}}\right) \phi^{-} \downarrow=\left(c^{\prime} \theta^{-}\right) \phi^{-} \downarrow=c^{\prime} \lambda^{-}=a .
$$

Thus, we have that $\left(\overline{R^{\prime}} \theta^{-}\right) \phi^{-} \downarrow$ is a $\Sigma_{0}^{-}$-message, and we have that $\left(\bar{R}^{\prime} \theta^{-}\right) \phi^{-} \downarrow=\left(R^{\prime} \theta^{-}\right) \phi^{-} \downarrow$, since the $\Sigma_{0}^{+}$-recipes $\overline{R^{\prime}}$ and $R^{\prime}$ coincide on their first argument.

In both cases, we have seen that $\left(\overline{R^{\prime}} \theta^{-}\right) \phi^{-} \downarrow=\left(R^{\prime} \theta^{-}\right) \phi^{-} \downarrow$, and also that $\mu_{\phi_{S}^{-}}^{1}\left(\overline{R^{\prime}}\right)<\mu_{\phi_{S}^{-}}^{1}\left(R^{\prime}\right)$. Since we know that $R^{\prime} \phi_{S}^{-} \downarrow$ is not a $\Sigma_{0}^{+}$-message, Lemma 4.6 applies, and we obtain that $\mu_{\phi_{S}^{-}}^{1}\left(R_{S}\left[\overline{R^{\prime}}\right]_{p}\right)<$ $\mu_{\phi_{S}^{-}}^{1}\left(R_{S}\left[R^{\prime}\right]_{p}\right)=\mu_{\phi_{S}^{-}}^{1}\left(R_{S}\right)$, and this contradicts the minimality of $R_{S}$.

Step 2: We now prove that $R_{S}$ is a simple $\Sigma_{0}^{+}$-recipe, in normal form w.r.t. $\downarrow$, and of the form $C\left[R_{1}, \ldots, R_{n}\right]$ where for each $i \in\{1, \ldots, n\}$, we have that $R_{i} \phi_{S}^{-} \downarrow$ is either an encrypted term, or a name from $\mathcal{N}$, or a constant from $\Sigma_{0}^{+}$.

Assume $R_{S}$ is not in normal form w.r.t. $\downarrow$. By Lemma 4.7, we know that $\mu_{\phi_{S}^{-}}^{1}\left(R_{S} \downarrow\right)<\mu_{\phi_{S}^{-}}^{1}\left(R_{S}\right)$. Moreover, as $R_{S} \theta^{-} \rightarrow^{*} R_{S} \Downarrow \theta^{-}$, Lemma 4.3 applies: $\left(R_{S} \Downarrow \theta^{-}\right) \phi^{-} \downarrow=\left(R_{S} \theta^{-}\right) \phi^{-} \downarrow$, and this contradicts the minimality of $R_{S}$. Therefore, we know that $R_{S}$ is in normal form w.r.t. $\downarrow$, and thus $R_{S}$ is a simple $\Sigma_{0}^{+}$-recipe, thanks to Lemma 4.5. Therefore, we have that $R_{S}=C\left[R_{1}, \ldots, R_{n}\right]$ where each $R_{i}$ is a subterm recipe with $1 \leq i \leq n$. If there is $i_{0} \in\{1, \ldots, n\}$ such that $\operatorname{root}\left(R_{i_{0}} \phi_{S}^{-} \downarrow\right)=$ $\mathrm{f} \in \Sigma_{\mathrm{c}}$ and $\mathrm{f}$ is a transparent function symbol, then $R_{i_{0}} \phi_{S}^{-} \downarrow=\mathrm{f}\left(C_{1}^{\mathrm{f}}\left[R_{i_{0}}\right], \ldots, C_{k}^{\mathrm{f}}\left[R_{i_{0}}\right]\right) \phi_{S}^{-} \downarrow$. We consider the context $\bar{C}$ such that $\bar{C}\left[R_{1}, \ldots, R_{n}\right]=C\left[R_{1}, \ldots, \mathrm{f}\left(C_{1}^{\mathrm{f}}\left[R_{i_{0}}\right], \ldots, C_{k}^{\mathrm{f}}\left[R_{i_{0}}\right]\right), \ldots, R_{n}\right]$. We have that $R_{S}^{\prime}=\bar{C}\left[R_{1}, \ldots, R_{n}\right]$ is a $\Sigma_{0}^{+}$-recipe such that $R_{S}^{\prime} \phi_{S}^{-} \downarrow=R_{S} \phi_{S}^{-} \downarrow$, and thus $\mu_{\phi_{S}}^{1}\left(R_{S}\right)=\mu_{\phi_{S}}^{1}\left(R_{S}^{\prime}\right)$, and $\left(R_{S}^{\prime} \theta^{-}\right) \phi^{-} \downarrow=R_{S}^{\prime} \phi_{S}^{-} \downarrow \lambda^{-}$by Lemma 4.9, which gives $\left(R_{S}^{\prime} \theta^{-}\right) \phi^{-} \downarrow=R_{S}^{\prime} \phi_{S}^{-} \downarrow \lambda^{-}=R_{S} \phi_{S}^{-} \downarrow \lambda^{-}=$ $\left(R_{S} \theta^{-}\right) \phi^{-} \downarrow$. We have that $\mu_{\phi_{S}}^{2}\left(R_{S}^{\prime}\right)<\mu_{\phi_{S}}^{2}\left(R_{S}\right)$ so this contradicts the minimality of $R_{S}$. Therefore, we deduce that each $R_{i} \phi_{S} \downarrow$ (with $1 \leq i \leq n$ ) is either an encrypted term, a constant from $\Sigma_{0}^{+}$, or a name from $\mathcal{N}$.

Step 3. Let $\mathcal{P}_{S}^{+}=\mathcal{P}^{+}, \phi_{S}^{+}=\phi_{S}^{-}, \sigma_{S}^{+}=\left.\sigma_{S}\right|_{d o m\left(\sigma^{+}\right)}$, and $i_{S}^{+}=i_{S}^{-}$. We are going to show that there exists a $\Sigma_{0}^{+}$-recipe $\overline{R_{S}}$ such that: 


$$
\left(\mathcal{P}_{S}^{-} ; \phi_{S}^{-} ; \sigma_{S}^{-} ; i_{S}^{-}\right) \stackrel{\text { in }\left(c, \overline{R_{S}}\right)}{=}\left(\mathcal{P}_{S}^{+} ; \phi_{S}^{+} ; \sigma_{S}^{+} ; i_{S}^{+}\right)
$$

as well as two substitutions $\theta^{+}$and $\lambda^{+}$that satisfy all our requirements.

If $R_{S} \phi_{S}^{-} \downarrow=u \sigma_{S}^{+}$, then let $\overline{R_{S}}=R_{S}, \theta^{+}=\theta^{-}$, and $\lambda^{+}=\lambda^{-}$. To conclude, it remains to establish that all our requirements are satisfied. In particular, we have to show that $(i)\left(\overline{R_{S}} \theta^{+}\right) \phi^{+} \downarrow=R \phi^{+} \downarrow$, and (ii) $\sigma^{+}=\sigma_{S}^{+} \lambda^{+}$.

(i) We have that $\left(\overline{R_{S}} \theta^{+}\right) \phi^{+} \downarrow=\left(R_{S} \theta^{-}\right) \phi^{-} \downarrow=u \sigma^{+}=R \phi^{-} \downarrow=R \phi^{+} \downarrow$.

(ii) Let $Z=\operatorname{vars}\left(u \sigma^{-}\right)=\operatorname{dom}(\tau)$. We have that $\overline{R_{S}} \phi_{S}^{-} \downarrow=u \sigma_{S}^{+}=u\left(\left.\sigma_{S}^{-} \uplus \sigma_{S}\right|_{Z}\right)$ is a $\Sigma_{0}^{+}$-message, and $\left(R_{S} \theta^{-}\right) \phi^{-} \downarrow=R \phi^{-} \downarrow=u \sigma^{+}=u\left(\left.\sigma^{-} \uplus \sigma\right|_{Z}\right)$. By Lemma 4.9, we know that $\overline{R_{S}} \phi_{S}^{-} \downarrow \lambda^{-}=$ $\left(R_{S} \theta^{-}\right) \phi^{-} \downarrow$, and thus $u\left(\left.\sigma_{S}^{-} \lambda^{-} \uplus \sigma_{S}\right|_{Z} \lambda^{-}\right)=u\left(\left.\sigma^{-} \uplus \sigma\right|_{Z}\right)$. This allows us to conclude that $\left.\sigma_{S}\right|_{Z} \lambda^{-}=\left.\sigma\right|_{Z}$, and thus we have that:

$$
\sigma_{S}^{+} \lambda^{+}=\left(\left.\sigma_{S}^{-} \uplus \sigma_{S}\right|_{Z}\right) \lambda^{-}=\left.\sigma_{S}^{-} \lambda^{-} \uplus \sigma_{S}\right|_{Z} \lambda^{-}=\left.\sigma^{-} \uplus \sigma\right|_{Z}=\sigma^{-} \uplus \tau=\sigma^{+} .
$$

Therefore, from now on, we assume that $R_{S} \phi_{S}^{-} \downarrow \neq u \sigma_{S}^{+}$. Let $A$ be the set of fresh constants that occur until this execution step, i.e., $A=\operatorname{St}\left(\operatorname{img}\left(\sigma_{S}^{+}\right)\right) \cap \sum_{\text {fresh }}$. We define $\lambda^{+}=\left.\lambda\right|_{A}$. As $\sigma=\sigma_{S} \lambda$, we deduce $\sigma^{+}=\sigma_{S}^{+} \lambda^{+}$. Since $R_{S} \phi_{S}^{-} \downarrow$ is a $\Sigma_{0}^{+}$-message, relying on Lemma 4.9, we deduce that $R_{S} \phi_{S}^{-} \downarrow \lambda^{+}=$ $R_{S} \phi_{S}^{-} \downarrow \lambda^{-}=\left(R_{S} \theta^{-}\right) \phi^{-} \downarrow=R \phi^{-} \downarrow=u \sigma^{+}=\left(u \sigma_{S}^{+}\right) \lambda^{+}$. Let $t=R_{S} \phi_{S} \downarrow$ and $v=u \sigma_{S}^{+}$. We have that $t \neq v$ and $t \lambda^{+}=v \lambda^{+}$.

Since $t \neq v$, we know that there exists a position $p$ defined in $t$ and $v$ such that $\operatorname{root}\left(\left.t\right|_{p}\right) \neq$ $\operatorname{root}\left(\left.v\right|_{p}\right)$. Let $p$ be any position defined in $t$ and $v$ such that $\operatorname{root}\left(\left.t\right|_{p}\right) \neq \operatorname{root}\left(\left.v\right|_{p}\right)$. Since $t \lambda^{+}=v \lambda^{+}$ and $\operatorname{dom}\left(\lambda^{+}\right) \subseteq \Sigma_{\text {fresh }}$, we have that $\left.t\right|_{p} \in \Sigma_{\text {fresh }}$ or $\left.v\right|_{p} \in \Sigma_{\text {fresh }}$.

We first assume that there exists such a position $p$ that falls outside the context $C$. More precisely, we have that $p=p^{\prime} \cdot p^{\prime \prime}$ (with $p^{\prime}$ a strict prefix of $p$ ) and $\left.C\left[R_{1}, \ldots, R_{n}\right]\right|_{p^{\prime}}=R_{i_{0}}$ for some $i_{0} \in\{1, \ldots, n\}$. Therefore, since $R_{i_{0}}$ is a subterm-recipe, we know that $\left.t\right|_{p^{\prime}}=R_{i_{0}} \phi_{S}^{-} \downarrow$ is an encrypted subterm of $\phi_{S}^{-}$. Relying on Lemma 4.10 and denoting $\mathcal{K}_{S}^{-}=\left(\mathcal{P}_{S}^{-} ; \phi_{S}^{-} ; \sigma_{S}^{-} ; i_{S}^{-}\right)$, we have that:

- $\left.t\right|_{p^{\prime}} \in \operatorname{ESt}\left(\phi_{S}^{-}\right) \subseteq \operatorname{ESt}\left(\mathcal{K}_{S}^{-} \sigma_{S}^{-}\right) \subseteq \operatorname{ESt}\left(\mathcal{K}_{P} \sigma_{S}^{-}\right)=\operatorname{ESt}\left(\mathcal{K}_{P}\left(\left.m g u(\Gamma)\right|_{\operatorname{dom}\left(\sigma_{S}^{-}\right)} \rho\right)\right) \subseteq \operatorname{ESt}\left(\mathcal{K}_{P}\right) \sigma_{S}^{-}$.

- $\left.v\right|_{p^{\prime}}=\left.u \sigma_{S}^{+}\right|_{p^{\prime}} \in \operatorname{ESt}\left(\mathcal{K}_{P} \sigma_{S}^{+}\right) \subseteq E S t\left(\mathcal{K}_{P}\left(\left.m g u(\Gamma)\right|_{\operatorname{dom}\left(\sigma_{S}^{+}\right)}\right) \rho\right) \subseteq E S t\left(\mathcal{K}_{P}\right) \sigma_{S}^{+}$.

This allows us to conclude that there exist $t^{\prime}, v^{\prime} \in \operatorname{ESt}\left(\mathcal{K}_{0}\right)$ such that $t^{\prime} \sigma_{S}^{-}=\left.t\right|_{p^{\prime}}$, and $v^{\prime} \sigma_{S}^{+}=\left.v\right|_{p^{\prime}}$. Since $\left.t\right|_{p^{\prime}}$ is a $\Sigma_{0}^{+}$-message, we also know that $t^{\prime} \sigma_{S}^{+}=\left.t\right|_{p^{\prime}}$. Since $t \lambda^{+}=v \lambda^{+}$, we know that $\left.t\right|_{p^{\prime}} \lambda^{+}=$ $\left.v\right|_{p^{\prime}} \lambda^{+}$, and thus $\left(t^{\prime} \sigma_{S}^{+}\right) \lambda^{+}=\left(v^{\prime} \sigma_{S}^{+}\right) \lambda^{+}$. We have that $\left(t^{\prime} \sigma_{S}^{+}\right) \lambda^{+}=t^{\prime}\left(\sigma_{S}^{+} \lambda^{+}\right)$and also that $\left(v^{\prime} \sigma_{S}^{+}\right) \lambda^{+}=$ $v^{\prime}\left(\sigma_{S}^{+} \lambda^{+}\right)$. Since we have that $\sigma^{+}=\sigma_{S}^{+} \lambda^{+}$, we deduce that $t^{\prime} \sigma^{+}=v^{\prime} \sigma^{+}$, and thus $t^{\prime} \sigma=v^{\prime} \sigma$. By definition of $\sigma_{S}$, we have that $t^{\prime} \sigma_{S}=v^{\prime} \sigma_{S}$. We know that $t^{\prime} \sigma_{S}^{-}=\left.t\right|_{p^{\prime}}$, and since $\left.t\right|_{p^{\prime}}$ is ground, we deduce that $t^{\prime} \sigma_{S}=\left.t\right|_{p^{\prime}}$. Similarly, we have that $v^{\prime} \sigma_{S}^{+}=\left.v\right|_{p^{\prime}}$, and since $\left.v\right|_{p^{\prime}}$ is ground, we deduce that $v^{\prime} \sigma_{S}=\left.v\right|_{p^{\prime}}$. Thus, we have that $\left.t\right|_{p^{\prime}}=\left.v\right|_{p^{\prime}}$ leading to a contradiction, since we have assumed that $t$ and $v$ differ below the position $p^{\prime}$.

Now, we know that for any position $p$ defined in $t$ and $v$ such that $\operatorname{root}\left(\left.t\right|_{p}\right) \neq \operatorname{root}\left(\left.v\right|_{p}\right)$, we have that $\left.t\right|_{p}$ or $\left.v\right|_{p}$ is in $\sum_{\text {fresh }}$, and $p$ is a position of $C$. If $\left.t\right|_{p}=c \in \Sigma_{\text {fresh }}$, let $R_{S}^{\prime}=R_{S}\left[c \theta^{-}\right]_{p}$, we have that $\mu_{\phi_{S}^{-}}^{1}\left(R_{S}^{\prime}\right)<\mu_{\phi_{S}^{-}}^{1}\left(R_{S}\right)$, since $\mu_{\phi_{S}^{-}}^{1}\left(c \theta^{-}\right)<\mu_{\phi_{S}^{-}}^{1}\left(\left.R_{S}\right|_{p}\right)$ (note that $\left.R_{S}\right|_{p} \phi_{S}^{-} \downarrow=c$, whereas $\left(c \theta^{-}\right) \phi_{S}^{-} \downarrow$ is a $\Sigma_{0}^{+}$-message and it is an atom when $\left.c \in \sum_{\text {fresh }}^{\text {atom }}\right)$. Moreover, we have that $\left(R_{S}^{\prime} \theta^{-}\right) \phi^{-} \downarrow=\left(R_{S} \theta^{-}\right) \phi^{-} \downarrow$. This will contradict the minimality of $R_{S}$. Therefore, we have that $\left.t\right|_{p} \notin \Sigma_{\text {fresh }}$, and thus $\left.v\right|_{p}=c$ for some $c \in \sum_{\text {fresh. }}$.

Let $p_{1}, \ldots, p_{m}$ be the positions such that for each $i \in\{1, \ldots, m\}$, we have that $p_{i}$ is defined in both $t$ and $v$, and $\operatorname{root}\left(\left.t\right|_{p_{i}}\right) \neq \operatorname{root}\left(\left.v\right|_{p_{i}}\right)$. For each $i \in\{1, \ldots, m\}$, we know that $p_{i}$ is a position of $C$ such that $\left.t\right|_{p_{i}} \notin \Sigma_{\text {fresh }}$, and $\left.v\right|_{p_{i}} \in \Sigma_{\text {fresh. }}$. We denote $c_{\text {fresh }}^{i}$ the constant from $\Sigma_{\text {fresh }}$ such that $\left.v\right|_{p_{i}}=c_{\text {fresh }}^{i}$. 
Note that it may happen that $c_{\text {fresh }}^{i}=c_{\text {fresh }}^{j}$ for some $i \neq j$. Let $\bar{C}$ be the context obtained from $C$ by putting $c_{\text {fresh }}^{1}$ at position $p_{1}, c_{\text {fresh }}^{2}$ at position $p_{2}, \ldots$ Let $\overline{R_{S}}=\bar{C}\left[R_{1}, \ldots, R_{n}\right]$. We have that $\overline{R_{S}} \phi_{S}^{-} \downarrow=u \sigma_{S}^{+}$by construction. Let $R_{p_{i}}=\left(\left.C\left[R_{1}, \ldots, R_{n}\right]\right|_{p_{i}}\right) \theta^{-}$for each $i \in\{1, \ldots, m\}$. Thanks to Lemma 4.9, we have that $R_{p_{i}} \phi^{-} \downarrow=R_{p_{i}} \phi_{S}^{-} \downarrow \lambda^{-}$for each $i \in\{1, \ldots, m\}$. As for each $i \in\{1, \ldots, m\}$, we have that $R_{p_{i}} \phi_{S}^{-} \downarrow \lambda^{-}=\left.u \sigma_{S}^{+} \lambda^{+}\right|_{p_{i}}$, we get that $R_{p_{i}} \phi_{S}^{-} \downarrow \lambda^{-}=c_{\text {fresh }}^{i} \lambda^{+}$for each $i \in\{1, \ldots, m\}$. Note that in case $c_{\text {fresh }}^{i}=c_{\text {fresh }}^{j}$, we have that $R_{p_{i}} \phi^{-} \downarrow=R_{p_{j}} \phi^{-} \downarrow$. Let $\theta^{+}=\theta^{-} \uplus\left\{c_{\text {fresh }}^{i} \mapsto R_{p_{i}} \mid c_{\text {fresh }}^{i} \notin \operatorname{dom}\left(\theta^{-}\right)\right\}$. In case we have that $c_{\text {fresh }}^{i}=c_{\text {fresh }}^{j}$ for some $i \neq j$, we choose arbitrarily between $R_{p_{i}}$ and $R_{p_{j}}$.

Now, it remains to establish that all our requirements are satisfied. In particular, we have to show that:

(i) $\sigma_{S}^{+}=\sigma_{S}^{-} \uplus \tau_{S}$ for some $\tau_{S}$ such that $\operatorname{dom}\left(\tau_{S}\right)=\operatorname{vars}\left(u \sigma_{S}^{-}\right)$and $\overline{R_{S}} \phi_{S}^{-} \downarrow=\left(u \sigma_{S}^{-}\right) \tau_{S}$.

(ii) for any $c \in \operatorname{dom}\left(\theta^{+}\right) \backslash \operatorname{dom}\left(\theta^{-}\right),\left(c \theta^{+}\right) \phi_{S}^{-} \downarrow$ is a $\Sigma_{0}^{+}$-message, and it is an atom when $c \in$ $\sum_{\text {fresh }}^{\text {atom }}$

(iii) for any $c \in \operatorname{dom}\left(\theta^{+}\right) \backslash \operatorname{dom}\left(\theta^{-}\right)$, we have that $c \lambda^{+}=\left(c \theta^{+}\right)\left(\phi_{S}^{-} \lambda^{-}\right) \downarrow$.

(iv) $\left(\overline{R_{S}} \theta^{+}\right) \phi^{+} \downarrow=R \phi^{+} \downarrow$.

We prove each item one-by-one.

(i) Let $\tau_{S}=\left.\sigma_{S}\right|_{\operatorname{dom}(\tau)}$. We have that $\operatorname{dom}\left(\tau_{S}\right)=\operatorname{dom}(\tau)=\operatorname{vars}\left(u \sigma^{-}\right)=\operatorname{vars}\left(u \sigma_{S}^{-}\right)$. We have shown that $\overline{R_{S}} \phi_{S}^{-} \downarrow=u \sigma_{S}^{+}$, and thus $\overline{R_{S}} \phi_{S}^{-} \downarrow=u\left(\sigma_{S}^{-} \uplus \tau_{S}\right)=\left(u \sigma_{S}^{-}\right) \tau_{S}$.

(ii) Let $c \in \operatorname{dom}\left(\theta^{+}\right) \backslash \operatorname{dom}\left(\theta^{-}\right)$. We have that $c=c_{\text {fresh }}^{i}$ for some $i \in\{1, \ldots, m\}$, and $\left(c_{\text {fresh }}^{i} \theta^{+}\right) \phi_{S}^{-} \downarrow=R_{p_{i}} \phi_{S}^{-} \downarrow$ is a $\Sigma_{0}^{+}$-message. Assume that $c_{\text {fresh }}^{i} \in \Sigma_{\text {fresh }}^{\text {atom }}$. In such a case, there exists $x_{i} \in \operatorname{dom}(\rho)$ such that $x_{i} \rho=c_{\text {fresh. }}^{i}$. As $x_{i} \rho$ is atomic, we know that $x_{i} \sigma$ is atomic (by definition of $\rho$ ), and we have that $x_{i} \sigma=x_{i} \sigma_{S} \lambda$. Since $\sigma_{S}=m g u(\Gamma) \rho$, we have that $x_{i} \sigma=x_{i} m g u(\Gamma) \rho \lambda$. As $x_{i} \in \operatorname{dom}(\rho), x_{i} \notin \operatorname{dom}(m g u(\Gamma))$ by definition of $\rho$. So $x_{i} \sigma=x_{i} \rho \lambda=c_{\text {fresh }}^{i} \lambda=c_{\text {fresh }}^{i} \lambda^{+}$. Since we have shown that $x_{i} \sigma$ is atomic, we deduce that $c_{\text {fresh }}^{i} \lambda^{+}$is atomic. Thus, since we have shown that $R_{p_{i}} \phi_{S}^{-} \downarrow \lambda^{-}=c_{\text {fresh }}^{i} \lambda^{+}$, we know that $R_{p_{i}} \phi_{S}^{-} \downarrow \lambda^{-}$is atomic, which implies that $R_{p_{i}} \phi_{S}^{-} \downarrow$ is either atomic or a $c \in \sum_{\text {fresh }}$ with $c \in \operatorname{dom}\left(\lambda^{-}\right)$. So, we can assume $c \in \sum_{\text {fresh }}$ with $c \in \operatorname{dom}\left(\lambda^{-}\right)$. There is an $x \in \operatorname{dom}(\rho)$ such that $x \rho=c$. We have $x \sigma=x \sigma_{S} \lambda=x m g u(\Gamma) \rho \lambda$. As $x \in \operatorname{dom}(\rho), x \notin \operatorname{dom}(m g u(\Gamma))$, and $x \sigma=x \rho \lambda=c \lambda=c \lambda^{-}$. As $x \sigma$ is atomic, $x \rho$ is atomic (by definition of $\rho$ ), so $c$ is atomic, and thus $R_{p_{i}} \phi_{S}^{-} \downarrow$ is atomic.

(iii) Let $c \in \operatorname{dom}\left(\theta^{+}\right) \backslash \operatorname{dom}\left(\theta^{-}\right)$. We have that $c=c_{\text {fresh }}^{i}$ for some $i \in\{1, \ldots, m\}$, and $c_{\text {fresh }}^{i} \lambda^{+}=$ $R_{p_{i}} \phi^{-} \downarrow=R_{p_{i}}\left(\phi_{S}^{-} \lambda^{-}\right) \downarrow=\left(c_{\text {fresh }}^{i} \theta^{+}\right)\left(\phi_{S}^{-} \lambda^{-}\right) \downarrow$.

(iv) $\left(\overline{R_{S}} \theta^{+}\right) \phi^{+} \downarrow=\left(R_{S} \theta^{-}\right) \phi^{-} \downarrow=R \phi^{-} \downarrow=R \phi^{+} \downarrow$.

This concludes the case where $\alpha$ is an input, and we get the result.

We can conclude with the proof of our main theorem on reachability properties.

THEOREM 3.8. Let $\mathcal{K}_{P}$ be a $\Sigma_{0}^{-}$-configuration type-compliant w.r.t. $\left(\mathcal{T}_{0}, \delta_{0}\right)$. If $\mathcal{K}_{P} \stackrel{\operatorname{tr}}{\Longrightarrow}(\mathcal{P} ; \phi ; \sigma ; i)$ w.r.t. $\Sigma_{0}^{-}$, then there exists a well-typed execution $\mathcal{K}_{P} \stackrel{\operatorname{tr}^{\prime}}{\Longrightarrow}\left(\mathcal{P} ; \phi^{\prime} ; \sigma^{\prime} ; i\right)$ w.r.t. $\Sigma_{0}^{+}$such that $\overline{\operatorname{tr}^{\prime}}=\overline{\operatorname{tr}}$.

Conversely, if $\mathcal{K}_{P} \stackrel{\operatorname{tr}^{\prime}}{\Longrightarrow}\left(\mathcal{P} ; \phi^{\prime} ; \sigma^{\prime} ; i\right)$ is a well-typed execution w.r.t. $\Sigma_{0}^{+}$, then there exists $\mathcal{K}_{P} \stackrel{\operatorname{tr}}{\Longrightarrow}$ $(\mathcal{P} ; \phi ; \sigma ; i)$ w.r.t. $\Sigma_{0}^{-}$such that $\overline{\mathrm{tr}}=\overline{\mathrm{tr}^{\prime}}$.

Proof. As explained in Section 3.3.1, the converse part is obvious. Thus, we now prove the direct part. Let $\mathcal{K}_{P}$ be a $\Sigma_{0}^{-}$-configuration type-compliant w.r.t. $\left(\mathcal{T}_{0}, \delta_{0}\right)$. Assume $\mathcal{K}_{P} \stackrel{\operatorname{tr}}{\Longrightarrow}(\mathcal{P} ; \phi ; \sigma ; i)$ 
w.r.t. $\Sigma_{0}^{-}$. Thanks to Proposition 4.11 , we know that there exists $\left(\operatorname{tr}_{S}, \phi_{S}\right) \in \operatorname{trace}_{\Sigma_{0}^{+}}\left(\mathcal{K}_{P}\right)$ with underlying substitution $\sigma_{S}$ with $\operatorname{dom}\left(\sigma_{S}\right)=\operatorname{dom}(\sigma)$ such that $\sigma_{S}=m g u(\Gamma) \rho$, as well as two substitutions $\lambda$ and $\theta$ such that:

- $\Gamma=\left\{(u, v) \mid u, v \in E S t\left(\mathcal{K}_{P}\right)\right.$ such that $\left.u \sigma=v \sigma\right\}$.

- $\rho$ is a bijective renaming from variables in $\operatorname{dom}(\sigma) \backslash \operatorname{dom}(m g u(\Gamma))$ to constants in $\sum_{\text {fresh }}$ such that $x \rho \in \Sigma_{\text {fresh }}^{\text {atom }}$ if, and only if, $x \sigma$ is an atomic $\Sigma_{0}^{-}$-message.

- $\operatorname{dom}(\theta) \subseteq \sum_{\text {fresh }}$, for any $c \in \sum_{\text {fresh }}$ occurring in $\operatorname{tr}_{S}$, we have that $c \in \operatorname{dom}(\theta), c \theta \in$ $\mathcal{T}\left(\Sigma, \Sigma_{0}^{-} \uplus \operatorname{dom}\left(\phi_{S}\right)\right)$, and $\mathrm{w}^{\prime} \prec c$ for any $\mathrm{w}^{\prime} \in \operatorname{vars}(c \theta)$ (where $<$ is the ordering induced by $\left.\operatorname{tr}_{S}\right)$.

- for any $c \in \operatorname{dom}(\theta),(c \theta) \phi_{S} \downarrow$ is a $\Sigma_{0}^{+}$-message, and it is an atom when $c \in \sum_{\text {fresh }}^{\text {atom }}$.

- $\lambda$ is the first-order substitution associated to $\theta$ through $\phi_{S}$.

- $\phi=\phi_{S} \lambda, \sigma=\sigma_{S} \lambda$, and $\left(\operatorname{tr}_{S} \theta\right) \phi \downarrow=\operatorname{tr} \phi \downarrow$.

Since $\left(\operatorname{tr}_{S} \theta\right) \phi \downarrow=\operatorname{tr} \phi \downarrow$, we have that $\overline{\operatorname{tr}_{S}}=\overline{\operatorname{tr}}$. Since we have enough constants of each type, we may assume w.l.o.g. that $\rho$ is well-typed. Since $\mathcal{K}_{P}$ is type-compliant, we know that encrypted subterms in $E S t\left(\mathcal{K}_{P}\right)$ that are unifiable have the same type. Then, by definition of a typing system, this allows us to deduce that $m g u(\Gamma)$ is well-typed, and thus $\sigma_{S}=m g u(\Gamma) \rho$ is well-typed. This means that $\left(\operatorname{tr}_{S}, \phi_{S}\right)$ is well-typed and concludes the proof.

\section{B PROOF OF SECTION 5}

The purpose of the lemma below is to lift a "symbolic" trace (i.e., a trace using special constants from $\Sigma_{\text {fresh }}$ ) into a concrete one. We are allowed to replace any constant from $\Sigma_{\text {fresh }}$ by any term, provided that such a term is deducible at the time we need it. Moreover, we have to pay attention to preserve atomicity. Under these conditions, we can show that the resulting trace is still a valid one.

Lemma B.1. Let $\mathcal{K}_{Q}=\left(Q_{0} ; \psi_{0} ; \sigma_{0} ; i_{0}\right)$ be a $\Sigma_{0}^{-}$-configuration, and $\left(\operatorname{tr}_{S}, \psi_{S}\right) \in \operatorname{trace}_{\Sigma_{0}^{+}}\left(\mathcal{K}_{Q}\right)$. Let $\theta$ be a substitution such that $\operatorname{dom}(\theta) \subseteq \sum_{\text {fresh }}$, for any $c \in \Sigma_{\text {fresh }}$ occurring in $\operatorname{tr}_{S}$, we have that $c \in \operatorname{dom}(\theta), c \theta \in \mathcal{T}\left(\Sigma, \Sigma_{0}^{-} \uplus \operatorname{dom}\left(\psi_{S}\right)\right)$, and $w^{\prime}<c$ for any $w^{\prime} \in \operatorname{vars}(c \theta)$ (where $<$ is the ordering induced by $\left.\operatorname{tr}_{S}\right)$. We also assume that $(c \theta) \psi_{S} \downarrow$ is a $\Sigma_{0}^{+}$-message for any $c \in \operatorname{dom}(\theta)$ and an atomic one in case $c \in \sum_{\text {fresh }}^{\mathrm{atom}}$. We have that $\left(\operatorname{tr}_{S} \theta, \psi_{S} \lambda\right) \in \operatorname{trace}_{\Sigma_{0}^{-}}\left(\mathcal{K}_{Q}\right)$ where $\lambda$ is the first-order substitution associated to $\theta$ through $\psi_{S}$.

Proof. Since $\left(\operatorname{tr}_{S}, \psi_{S}\right) \in \operatorname{trace}_{\Sigma_{0}^{+}}\left(\mathcal{K}_{Q}\right)$, we know that $\mathcal{K}_{Q}=\left(Q_{0} ; \psi_{0} ; \sigma_{0} ; i_{0}\right) \stackrel{\operatorname{tr}_{S}}{=}\left(Q_{S} ; \psi_{S} ; \sigma_{S} ; i_{S}\right)$. Given $\theta$ and $\lambda$ as defined in the lemma, we establish that: $\mathcal{K}_{Q}=\left(Q_{0} ; \psi_{0} ; \sigma_{0} ; i_{0}\right) \stackrel{\operatorname{tr}_{S} \theta}{\Longrightarrow}(Q ; \psi ; \sigma ; i)$ where $Q=Q_{S}, \phi=\psi_{S} \lambda, \sigma=\sigma_{S} \lambda$, and $i=i_{S}$. We show this result by induction on the length of the trace.

Base case: $\operatorname{tr}$ is empty. Let $(Q ; \psi ; \sigma ; i)=\mathcal{K}_{Q}$. Since $\mathcal{K}_{Q}$ is a $\Sigma_{0}^{-}$-configuration, we have that $\psi \lambda=\psi$, and $\sigma \lambda=\sigma$. Therefore, the result trivially holds.

Inductive case: $\operatorname{tr}=\operatorname{tr}^{-} . \alpha_{S}$. In such a case, we have that:

$$
\mathcal{K}_{Q}=\left(Q_{0} ; \psi_{0} ; \sigma_{0} ; i_{0}\right) \stackrel{\operatorname{tr} S}{\Longrightarrow}\left(Q_{S}^{-} ; \psi_{S}^{-} ; \sigma_{S}^{-} ; i_{S}^{-}\right) \stackrel{\alpha_{S}}{\Longrightarrow}\left(Q_{S} ; \psi_{S} ; \sigma_{S} ; i_{S}\right) .
$$

Thanks to our induction hypothesis applied on $\operatorname{tr}^{-}$, we know that:

$$
\mathcal{K}_{Q}=\left(Q_{0} ; \psi_{0} ; \sigma_{0} ; i_{0}\right) \stackrel{\operatorname{tr} \theta}{\Longrightarrow}\left(Q^{-} ; \psi^{-} ; \sigma^{-} ; i^{-}\right)
$$

with $Q^{-}=Q_{S}^{-}, \psi^{-}=\psi_{S}^{-} \lambda, \sigma^{-}=\sigma_{S}^{-} \lambda$, and $i^{-}=i_{S}^{-}$. We distinguish three cases depending on $\alpha_{S}$. 
- Case $\alpha_{S}=$ phase $j$ for some integer $j$. In such a case, we have that $Q_{S}=Q_{S}^{-}, \psi_{S}=\psi_{S}^{-}, \sigma_{S}=\sigma_{S}^{-}$, and $i_{S}=j>i_{S}^{-}$. Let $Q=Q^{-}, \psi=\psi^{-}, \sigma=\sigma^{-}, i=j$. Since $i^{-}=i_{S}^{-}<j$. We have that:

$$
\left(Q^{-} ; \psi^{-} ; \sigma^{-} ; i^{-}\right) \stackrel{\text { phase } j}{\longrightarrow}\left(Q^{-} ; \psi^{-} ; \sigma^{-} ; j\right)=(Q ; \psi ; \sigma ; i) .
$$

We have that $Q=Q_{S}, \psi=\psi_{S} \lambda, \sigma=\sigma_{S} \lambda$, and $i=i_{S}$. It gives us the result.

- Case $\alpha_{S}=\operatorname{in}(c, R)$ for some $\Sigma_{0}^{+}$-recipe $R$. In such a case, we have that $Q_{S}^{-}=\left\{\operatorname{in}(c, u) . Q_{c}\right\} \uplus \mathcal{P}$, and we have also that $u \sigma_{S}^{-}$and $R \psi_{S}^{-} \downarrow$ (ground term) are unifiable with some substitution $\tau$, and we have that $\sigma_{S}=\sigma_{S}^{-} \uplus \tau$. Thanks to Lemma 4.8, we can apply Lemma 4.9, and thus we have:

$$
(R \theta) \psi^{-} \downarrow=(R \theta)\left(\psi_{S} \lambda\right) \downarrow=\left(R \psi_{S}^{-} \downarrow\right) \lambda=\left[\left(u \sigma_{S}^{-}\right) \tau\right] \lambda=u\left(\sigma_{S}^{-} \uplus \tau\right) \lambda=\left(u\left(\sigma_{S}^{-} \lambda\right)\right)(\tau \lambda) .
$$

Let $Q=\left\{Q_{c}\right\} \uplus \mathcal{P}, \psi=\psi^{-}, \sigma=\sigma^{-} \uplus \tau \lambda$, and $i=i^{-}$. We have that:

$$
\left(\left\{\operatorname{in}(c, u) \cdot Q_{c}\right\} \uplus \mathcal{P} ; \psi^{-} ; \sigma^{-} ; i^{-}\right) \stackrel{\operatorname{in}(c, R \theta)}{\longrightarrow}\left(\left\{Q_{c}\right\} \uplus \mathcal{P} ; \psi^{-} ; \sigma^{-} \uplus \tau \lambda ; i^{-}\right)=(Q ; \psi ; \sigma ; i) .
$$

We have that $Q=Q_{S}, \psi=\psi_{S} \lambda, i=i_{S}$, and $\sigma=\sigma^{-} \uplus \tau \lambda=\sigma_{S}^{-} \lambda \uplus \tau \lambda=\left(\sigma_{S}^{-} \uplus \tau\right) \lambda=\sigma_{S} \lambda$. It gives us the result.

- Case $\alpha_{S}=\operatorname{out}(c, \mathrm{w})$. In such a case, we have that $Q_{S}^{-}=Q^{-}=\left\{\operatorname{out}(c, u) \cdot Q_{c}\right\} \uplus \mathcal{P}$, and we have that $\psi_{S}=\psi_{S}^{-} \uplus\left\{\mathrm{w} \triangleright u \sigma_{S}^{-}\right\}$. Let $Q=\left\{Q_{c}\right\} \uplus \mathcal{P}, \psi=\psi^{-} \uplus\left\{\mathrm{w} \triangleright u \sigma^{-}\right\}, \sigma=\sigma^{-}$, and $i=i^{-}$. We now show that $u \sigma^{-}=u \sigma_{S}^{-} \lambda$ is a $\Sigma_{0}^{-}$-message. We know that $u \sigma_{S}^{-}$is a $\Sigma_{0}^{+}$-message. Moreover, thanks to Lemma 4.8, we know that $c \lambda$ is a $\Sigma_{0}^{-}$-message for each $c \in \operatorname{dom}(\lambda)$, and $c \lambda$ is atomic when $c \in \sum_{\text {fresh }}^{\text {atom }}$. This allows us to conclude that $u \sigma^{-}=u \sigma_{S}^{-} \lambda$ is a $\Sigma_{0}^{-}$-message. Therefore, we have that:

$$
\left(\left\{\text { out }(c, u) . Q_{c}\right\} \uplus \mathcal{P} ; \psi^{-} ; \sigma^{-} ; i^{-}\right) \stackrel{\text { out }(c, \mathrm{w})}{\longrightarrow}\left(\left\{Q_{c}\right\} \uplus \mathcal{P} ; \psi^{-} \uplus\left\{\mathrm{w} \triangleright u \sigma^{-}\right\} ; \sigma^{-} ; i^{-}\right)=(Q ; \psi ; \sigma ; i) \text {. }
$$

We have that $Q=Q_{S}, \sigma=\sigma_{S} \lambda, i=i_{S}$, and $\psi=\psi_{S} \lambda$, since $\sigma_{S}^{-} \lambda=\sigma^{-}$by induction hypothesis. It gives us the result.

We may note that the resulting $\operatorname{trace} \operatorname{tr}_{S} \theta$ only contains $\Sigma_{0}^{-}$-recipes: constants from $\Sigma_{\text {fresh }}$ occurring in $\operatorname{tr}_{S}$ have been replaced by $\theta$. Hence, the result.

Proposition 5.4. Let $\mathcal{K}_{P}$ and $\mathcal{K}_{Q}$ be two initial $\Sigma_{0}^{-}$-configurations such that $\mathcal{K}_{P} \nsubseteq_{t} \mathcal{K}_{Q}$ w.r.t. $\Sigma_{0}^{-}$. Let $(\operatorname{tr}, \phi) \in \operatorname{trace}_{\Sigma_{0}^{-}}\left(\mathcal{K}_{P}\right)$ with underlying substitution $\sigma$ be a witness of non-inclusion. Then, there exists $\left(\operatorname{tr}_{S}, \phi_{S}\right) \in$ trace $_{\Sigma_{0}^{+}}\left(\mathcal{K}_{P}\right)$ a witness of this non-inclusion with underlying substitution $\sigma_{S}$ such that $\sigma_{S}=m g u(\Gamma) \rho$, as well as two substitutions $\lambda_{P}$ and $\theta$ such that $\left(\rho, \lambda_{P}, \theta\right)$ is a well-typed concretization of $\left(\operatorname{tr}_{S}, \phi_{S}\right)$ w.r.t. $(\operatorname{tr}, \phi)$ in the context $\mathcal{K}_{P}$, as defined in Proposition 4.11.

Proof. Let $(\operatorname{tr}, \phi) \in \operatorname{trace}_{\Sigma_{0}^{-}}\left(\mathcal{K}_{P}\right)$ be a witness of non-inclusion with underlying substitution $\sigma$. First, we apply Proposition 4.11. We deduce that there exists $\left(\operatorname{tr}_{S}, \phi_{S}\right) \in \operatorname{trace}_{\Sigma_{0}^{+}}\left(\mathcal{K}_{P}\right)$ with underlying substitution $\sigma_{S}$ with $\operatorname{dom}\left(\sigma_{S}\right)=\operatorname{dom}(\sigma)$ such that $\sigma_{S}=m g u(\Gamma) \rho$, as well as two substitutions $\lambda_{P}$ and $\theta$ such that:

- $\Gamma=\left\{(u, v) \mid u, v \in E S t\left(\mathcal{K}_{P}\right)\right.$ such that $\left.u \sigma=v \sigma\right\}$.

- $\rho$ is a bijective renaming from variables in $\operatorname{dom}(\sigma) \backslash \operatorname{dom}(m g u(\Gamma))$ to constants in $\Sigma_{\text {fresh }}$ such that $x \rho \in \Sigma_{\text {fresh }}^{\text {atom }}$ if, and only if, $x \sigma$ is an atomic $\Sigma_{0}^{-}$-message.

- $\operatorname{dom}(\theta) \subseteq \sum_{\text {fresh }}$, for any $c \in \sum_{\text {fresh }}$ occurring in $\operatorname{tr}_{S}$, we have that $c \in \operatorname{dom}(\theta), c \theta \in$ $\mathcal{T}\left(\Sigma, \Sigma_{0}^{-} \uplus \operatorname{dom}\left(\phi_{S}\right)\right)$, and $\mathrm{w}^{\prime} \prec c$ for any $\mathrm{w}^{\prime} \in \operatorname{vars}(c \theta)$ (where $\prec$ is the ordering induced by $\left.\operatorname{tr}_{S}\right)$.

- for any $c \in \operatorname{dom}(\theta),(c \theta) \phi_{S} \downarrow$ is a $\Sigma_{0}^{+}$-message, and it is an atom when $c \in \sum_{\text {fresh }}^{\text {atom }}$.

- $\lambda_{P}$ is the first-order substitution associated to $\theta$ through $\phi_{S}$.

- $\phi=\phi_{S} \lambda_{P}, \sigma=\sigma_{S} \lambda_{P}$, and $\left(\operatorname{tr}_{S} \theta\right) \phi \downarrow=\operatorname{tr} \phi \downarrow$. 
Our goal is to show that $\left(\operatorname{tr}_{S}, \phi_{S}\right) \in \operatorname{trace}_{\Sigma_{0}^{+}}\left(\mathcal{K}_{P}\right)$ is a witness of $\mathcal{K}_{P} \nsubseteq_{t} \mathcal{K}_{Q}$ w.r.t $\Sigma_{0}^{+}$.

Let $\psi_{S}$ be such that $\left(\operatorname{tr}_{S}, \psi_{S}\right) \in \operatorname{trace}_{\Sigma_{0}^{+}}\left(\mathcal{K}_{Q}\right)$ and $\phi_{S} \sqsubseteq_{s} \psi_{S}$ (and thus $\left.\operatorname{dom}\left(\phi_{S}\right)=\operatorname{dom}\left(\psi_{S}\right)\right)$. Note that such a $\psi_{S}$ exists, since otherwise the result trivially holds.

We have that $\phi_{S}$ is a $\Sigma_{0}^{+}$-frame and $\prec$ is an ordering on $\operatorname{dom}\left(\phi_{S}\right)$. We have $\operatorname{dom}(\theta) \subseteq \Sigma_{\text {fresh }}$ and $c \theta \in \mathcal{T}\left(\Sigma, \Sigma_{0}^{-} \uplus \operatorname{dom}\left(\phi_{S}\right)\right)$ for any $c \in \operatorname{dom}(\theta)$. Moreover, if $c \in \Sigma_{\text {fresh }}$ occurs in $\mathrm{w} \phi_{S}$, then, as $c$ does not occur in $\mathcal{K}_{P}$, it must have been introduced in an input before the output of $\mathrm{w}$. So $c<\mathrm{w}$. Let $\mathrm{w}^{\prime} \in \operatorname{vars}(c \theta)$. Then $\mathrm{w}^{\prime}<c$ by definition of $<$. So $\mathrm{w}^{\prime}<\mathrm{w}$ and the order induced by $\prec$ on $\operatorname{dom}\left(\phi_{S}\right)$ satisfies the condition of Lemma 4.8 and Lemma 4.9. $(c \theta) \phi_{S} \downarrow$ is a $\Sigma_{0}^{+}$-message for each $c \in \operatorname{dom}(\theta)$ and $(c \theta) \phi_{S} \downarrow$ is an atomic $\Sigma_{0}^{+}$-message when $c \in \Sigma_{\text {fresh }}^{\text {atom }}$. So Lemma 4.8 applies and for each $c \in \operatorname{dom}\left(\lambda_{P}\right), c \lambda_{P}$ is a $\Sigma_{0}^{-}$-message and $c \lambda_{P}$ is atomic if $c \in \sum_{\text {fresh }}^{\text {atom }}$. So Lemma 4.9 applies, and for any recipe $R$ such that $R \phi_{S} \downarrow$ is a $\Sigma_{0}^{+}$-message, we have $(R \theta)\left(\phi_{S} \lambda_{P}\right) \downarrow=\left(R \phi_{S} \downarrow\right) \lambda_{P}$.

$\mathcal{K}_{Q}$ is a $\Sigma_{0}^{-}$-configuration, and $\left(\operatorname{tr}_{S}, \psi_{S}\right) \in \operatorname{trace}_{\Sigma_{0}^{+}}\left(\mathcal{K}_{Q}\right)$. Moreover, $\operatorname{dom}(\theta) \subseteq \Sigma_{\text {fresh }}$, and $c \in$ $\operatorname{dom}(\theta)$ for each $c$ occurring in $\operatorname{tr}_{S}$. We have $\operatorname{dom}\left(\phi_{S}\right)=\operatorname{dom}\left(\psi_{S}\right)$ by $\phi_{S} \sqsubseteq_{s} \psi_{S}$, so for each $c \in$ $\operatorname{dom}(\theta), c \theta \in \mathcal{T}\left(\Sigma, \Sigma_{0}^{-} \uplus \operatorname{dom}\left(\phi_{S}\right)\right) \subseteq \mathcal{T}\left(\Sigma, \Sigma_{0}^{-} \uplus \operatorname{dom}\left(\psi_{S}\right)\right)$. Moreover, if $c \in \Sigma_{\text {fresh }}$ occurs in $\mathrm{w} \psi_{S}$, then, as $c$ does not occur in the protocol, it must have been introduced in an input before the output of $\mathrm{w}$. So $c<\mathrm{w}$. Let $\mathrm{w}^{\prime} \in \operatorname{vars}(c \theta)$. Then $\mathrm{w}^{\prime}<c$ by definition of $<$. So $\mathrm{w}^{\prime}<\mathrm{w}$ and the order induced by $\prec$ on $\operatorname{dom}\left(\psi_{S}\right)$ satisfies the condition of Lemma 4.8 and Lemma 4.9. By $\phi_{S} \sqsubseteq_{s} \psi_{S},(c \theta) \psi_{S} \downarrow$ is a $\Sigma_{0}^{+}$-message for any $c \in \operatorname{dom}(\theta)$ and an atomic one in case $c \in \Sigma_{\text {fresh }}^{\text {atom }}$. Hence, we have that both Lemma B.1 and Lemma 4.8 apply. We obtain that $\left(\operatorname{tr}_{S} \theta, \psi_{S} \lambda_{Q}\right) \in \operatorname{trace}_{\Sigma_{0}^{-}}\left(\mathcal{K}_{Q}\right)$ where $\lambda_{Q}$ is the first-order substitution associated to $\theta$ through $\psi_{S}$. We also get that $c \lambda_{Q}$ is a $\Sigma_{0}^{-}$-message for each $c \in \operatorname{dom}\left(\lambda_{Q}\right)$, and it is atomic when $c \in \sum_{\text {fresh }}^{\text {atom }}$. Therefore, Lemma 4.9 applies, and we have that for any $R \in \mathcal{T}\left(\Sigma, \Sigma_{0}^{-} \uplus \operatorname{dom}(\theta) \uplus \operatorname{dom}\left(\psi_{S}\right)\right)$ such that $R \psi_{S} \downarrow$ is a $\Sigma_{0}^{+}$-message, $(R \theta)\left(\psi_{S} \lambda_{Q}\right) \downarrow=\left(R \psi_{S} \downarrow\right) \lambda_{Q}$. Moreover, since $\lambda_{Q}$ preserves atomicity, $\left(R \psi_{S} \downarrow\right) \lambda_{Q}$ is a $\Sigma_{0}^{-}$-message whenever $\left(R \psi_{S} \downarrow\right)$ is a $\Sigma_{0}^{+}-$ message.

In the remaining of this proof, we suppose that $\phi_{S} \sqsubseteq_{s} \psi_{S}$ (meaning that $\left(\operatorname{tr}_{S}, \phi_{S}\right) \in \operatorname{trace}_{\Sigma_{0}^{+}}\left(\mathcal{K}_{P}\right)$ is not a witness), and we show that $\phi \sqsubseteq_{s} \psi$ leading to a contradiction, since by hypothesis, we know that $(\operatorname{tr}, \phi) \in \operatorname{trace}_{\Sigma_{0}^{-}}\left(\mathcal{K}_{P}\right)$ is a witness, and tr passes in $\mathcal{K}_{Q}$ leading to the frame $\psi$.

To establish this result, we rely on Lemma 5.3 and thus, we reason with the notion $\sqsubseteq_{s}^{\text {atom }}$. We consider a test $T$ (built on $\mathcal{T}\left(\Sigma, \Sigma_{0}^{+} \cup \operatorname{dom}\left(\phi_{S}\right)\right)$ such that $T \theta$ holds in $\phi$. We assume that for all $T^{\prime}$ such that $\mu_{\phi_{S}}\left(T^{\prime}\right)<\mu_{\phi_{S}}(T)$, we have that:

\section{$T^{\prime} \theta$ holds in $\phi$ implies that $T^{\prime} \theta$ holds in $\psi$.}

We have to prove that $T \theta$ holds in $\psi$. We distinguish three cases depending on the form of the test:

(1) The test $T$ is a $\Sigma_{0}^{+}$-recipe $R$ such that $(R \theta) \phi \downarrow$ is a $\Sigma_{0}^{-}$-message. In such a case, we have to establish that $(R \theta) \psi \downarrow$ is a $\Sigma_{0}^{-}$-message

(2) The test $T$ is a $\Sigma_{0}^{+}$-recipe such that $(R \theta) \phi \downarrow$ is an atomic $\Sigma_{0}^{-}$-message, i.e., $(R \theta) \phi \downarrow \in \Sigma_{0}^{-} \uplus \mathcal{N}$. In such a case, we have to establish that $(R \theta) \psi \downarrow$ is an atomic $\Sigma_{0}^{-}$-message.

(3) The test $T$ is made of two $\Sigma_{0}^{+}$-recipes $R, R^{\prime}$ such that both $(R \theta) \phi \downarrow$ and $\left(R^{\prime} \theta\right) \phi \downarrow$ are $\Sigma_{0}^{-}$-messages, and $(R \theta) \phi \downarrow=\left(R^{\prime} \theta\right) \phi \downarrow$. In such a case, we have to establish that $(R \theta) \psi \downarrow=$ $\left(R^{\prime} \theta\right) \psi \downarrow$.

(1) $R$ is $\Sigma_{0}^{+}$-recipe such that $(R \theta) \phi \downarrow$ is a $\Sigma_{0}^{-}$-message.

Assume that $R \phi_{S} \downarrow$ is not a $\Sigma_{0}^{+}$-message. We take the smallest subterm $R^{\prime}$ of $R$ such that $R^{\prime} \phi_{S} \downarrow$ is not a $\Sigma_{0}^{+}$-message. Let $p$ be such that $\left.R\right|_{p}=R^{\prime}$. As $R^{\prime} \phi_{S} \downarrow$ is not a $\Sigma_{0}^{+}$-message, we know that 
$R^{\prime} \notin \Sigma_{0}^{+} \uplus \operatorname{dom}\left(\phi_{S}\right)$. Thus, we have that $R^{\prime}=\mathrm{f}\left(R_{1}, \ldots, R_{k}\right)$ for some $\mathrm{f} \in \Sigma$. Moreover, by minimality of $R^{\prime}$, we know that $R_{i} \phi_{S} \downarrow$ is a $\Sigma_{0}^{+}$-message for $1 \leq i \leq k$. We now establish the following claim:

CLAIM. If $R_{i} \phi_{S} \downarrow \in \sum_{\text {fresh }}$ for some $i \in\{1, \ldots, k\}$, then $(R \theta) \psi \downarrow$ is a $\Sigma_{0}^{-}$-message.

Proof. Assume $R_{i} \phi_{S} \downarrow=c \in \sum_{\text {fresh }}$ for some $i \in\{1, \ldots, k\}$, and let $R_{i}^{\prime}=c \theta$. We have that $R_{i}^{\prime} \theta=$ $c \theta$, and thus $\left(R_{i}^{\prime} \theta\right) \phi \downarrow=(c \theta) \phi \downarrow$, and $\left(R_{i}^{\prime} \theta\right) \psi \downarrow=(c \theta) \psi \downarrow$. We have that the test $c=R_{i}$ holds in $\phi_{S}$, and thus, since $\phi_{S} \sqsubseteq_{s} \psi_{S}$, it also holds in $\psi_{S}$. Lemma 4.9 applies, and we obtain: $\left(R_{i} \theta\right) \psi \downarrow=\left(R_{i} \psi_{S} \downarrow\right) \lambda_{Q}=$ $c \lambda_{Q}=(c \theta) \psi \downarrow=\left(R_{i}^{\prime} \theta\right) \psi \downarrow$. Let $\bar{R}=R\left[R_{i}^{\prime}\right]_{(p . i)}$. We have that:

- $(\bar{R} \theta=R \theta)$ holds in both $\phi$ and $\psi$;

- $\mu_{\phi_{S}}^{1}\left(R_{i}^{\prime}\right)<\mu_{\phi_{S}}^{1}\left(R_{i}\right)$, thanks to Lemma A.4, and thus $\mu_{\phi_{S}}^{1}(\bar{R})<\mu_{\phi_{S}}^{1}(R)$, thanks to Lemma A.3 and Lemma 4.6.

As $(\bar{R} \theta) \phi \downarrow=(R \theta) \phi \downarrow$ is a $\Sigma_{0}^{-}$-message, such a test transfers to $\psi$ (relying on our induction hypothesis), and $(\bar{R} \theta) \psi \downarrow$ is a $\Sigma_{0}^{-}$-message. It proves our claim.

We now distinguish two cases depending on whether $f \in \Sigma_{\mathrm{c}}$ or $\mathrm{f} \in \Sigma_{\mathrm{d}}$.

Case where $f \in \Sigma_{\mathrm{c}}$. In such a case, $R^{\prime} \phi_{S} \downarrow$ is either not well-shaped or not well-sorted. If it is not well-sorted, then for one of its subrecipes $R_{i}, R_{i} \phi_{S} \downarrow$ is not an atom (it is well-sorted by minimality of $R^{\prime}$ ) while it should be. In particular, $\left(R_{i} \theta\right) \phi \downarrow=R_{i} \phi_{S} \downarrow \lambda_{P}$ is an atom. So $R_{i} \phi_{S} \downarrow$ must be in $\Sigma_{\text {fresh }}$, which implies by our claim that $(R \theta) \psi \downarrow$ is a $\Sigma_{0}^{-}$-message, and thus we are done. We deduce that $R^{\prime} \phi_{S} \downarrow$ is well-sorted.

Now, we assume that $R^{\prime} \phi_{S} \downarrow$ is not well-shaped, we consider the shape of $\mathrm{f}, s h_{\mathrm{f}}=\mathrm{f}\left(s_{1}, \ldots, s_{k}\right)$ for some $s_{1}, \ldots, s_{k}$. As $R^{\prime} \phi_{S} \downarrow$ has a bad shape and is a f-term, there must be a $j$ such that $R_{j} \phi_{S} \downarrow$ is not an instance of $s_{j}$. In particular, $s_{j}$ is not a variable and we have $s_{j}=s h_{\mathrm{g}}$ for some function symbol $g$ (thanks to the compatibility of the shapes). But $R_{j} \phi_{S} \downarrow$ is a $\Sigma_{0}^{+}$-message, and thus we know that $\left(R_{j} \theta\right) \phi \downarrow=\left(R_{j} \phi_{S} \downarrow\right) \lambda_{P}$ (thanks to Lemma 4.9) is an instance of $s_{j}$, as $\left(R_{j} \theta\right) \phi \downarrow$ is a $\Sigma_{0}^{+}$-message. Relying on our claim, we know that $R_{j} \phi_{S} \downarrow \notin \Sigma_{\text {fresh }}$, thus $R_{j} \phi_{S} \downarrow$ is a g-term, and since we know that $R_{j} \phi_{S} \downarrow$ is a $\Sigma_{0}^{+}$-message, we know that it is an instance of $s_{j}$, yielding to a contradiction.

Case where $\mathrm{f}=\operatorname{des} \in \Sigma_{\mathrm{d}}$. In such a case, $R^{\prime}=\operatorname{des}\left(R_{1}, \ldots, R_{k}\right)$. Let $\ell_{\text {des }}=\operatorname{des}\left(t_{1}, t_{2}, \ldots, t_{k}\right)$. We distinguish two subcases.

First, we assume that there is some $i \in\{1, \ldots, k\}$ such that $R_{i} \phi_{S} \downarrow$ does not unify with $t_{i}$. As $R_{i} \phi_{S} \downarrow$ is a $\Sigma_{0}^{+}$-message, it has a good shape. Thus, the only way to not unify with the linear term $t_{i}$ whereas $\left(R_{i} \phi_{S}\right) \lambda_{P}=\left(R_{i} \theta\right) \phi \downarrow$ (thanks to Lemma 4.9) does, is when $R_{i} \phi_{S} \downarrow=c$ for some $c \in \Sigma_{\text {fresh. }}$. Relying on our claim, we obtain a contradiction.

Second, we assume that $R_{i} \phi_{S} \downarrow$ unifies with $t_{i}$ for each $i$. In such a case, we know that $\ell_{\text {des }}=$ $\operatorname{des}\left(t_{1}, \ldots, t_{k}\right)$ is a non-linear term and we denote $x$ the non-linear variable occurring in $\ell_{\text {des }}$. Let $I_{0}=\left\{1 \leq i \leq k \mid x\right.$ occurs in $\left.t_{i}\right\}$. We know that $1 \in I_{0}$. For any $i \in I_{0}$, we denote $p_{i}$ the position in $t_{i}$ such that $\left.t_{i}\right|_{p_{i}}=x$. Since $R^{\prime} \phi_{S} \downarrow \lambda_{P}$ is a $\Sigma_{0}^{-}$-message, we know that $t=\operatorname{des}\left(R_{1} \phi_{S} \downarrow \lambda_{P}, \ldots, R_{k} \phi_{S} \downarrow \lambda_{P}\right)$ unifies with $\operatorname{des}\left(t_{1}, \ldots, t_{k}\right)$. Therefore, we know that there exists an atomic $\Sigma_{0}^{-}$-message $a$ such that $\left.t\right|_{i . p_{i}}=a$ for any $i \in I_{0}$. We know that $R^{\prime} \phi_{S} \downarrow$ is not a $\Sigma_{0}^{+}$-message, whereas $R_{i} \phi_{S} \downarrow$ are $\Sigma_{0}^{+}$-messages. Thus, we have that $t_{S}=\operatorname{des}\left(R_{1} \phi_{S} \downarrow, \ldots, R_{k} \phi_{S} \downarrow\right)$ does not unify with $\operatorname{des}\left(t_{1}, \ldots, t_{k}\right)$. We deduce the following fact:

Fact: For any $i \in I_{0}$, we have $\left.t_{S}\right|_{i . p_{i}}=a$, or $\left.t_{S}\right|_{i . p_{i}}=c$ for some $c \in \sum_{\text {fresh }}$ such that $c \lambda_{P}=$ $(c \theta) \phi \downarrow=a$.

Let $c$ be a constant from $\Sigma_{\text {fresh }}$ such that $\left.t_{S}\right|_{i . p_{i}}=c$ for some $i \in I_{0}$. Let $I_{1}=\left\{i \in I_{0} \mid R_{i} \phi_{S} \downarrow_{p_{i}}=\right.$ c). We will build two recipes $\bar{R}^{\prime}$ and $\bar{R}^{\prime \prime}$ derived from $R^{\prime}$ and enjoying some nice properties: in 
particular, both $\left(\bar{R}^{\prime} \theta\right) \psi \downarrow$ and $\left(\bar{R}^{\prime \prime} \theta\right) \psi \downarrow$ will be $\Sigma_{0}^{-}$-message, and this will allow us to derive that $(R \theta) \psi \downarrow$ is a $\Sigma_{0}^{-}$-message, too.

Construction of $\overline{R^{\prime}}$. Let $v_{\text {des }}^{\prime}$ be the substitution such that $x v_{\text {des }}^{\prime}=c \theta$, and $y v_{\text {des }}^{\prime}=c_{\text {min }}$ for any other variable $y \in \operatorname{vars}\left(\ell_{\mathrm{des}}\right)$. For $i \in\{1, \ldots, k\}$, let $R_{i}^{\prime}=t_{i} v_{\text {des }}^{\prime}$ in case $i \in I_{1}$, and $R_{i}^{\prime}=R_{i}$ otherwise. Let $\bar{R}^{\prime}=\operatorname{des}\left(R_{1}^{\prime}, \ldots, R_{k}^{\prime}\right)$. Each $R_{i}^{\prime} \phi_{S} \downarrow$ is a $\Sigma_{0}^{+}$-message, and thus by Lemma 4.9 , we have that $\left(R_{i}^{\prime} \theta\right) \phi \downarrow=$ $R_{i}^{\prime} \phi_{S} \downarrow \lambda_{P}$ is a $\Sigma_{0}^{-}$-message. By construction of $\bar{R}^{\prime}$ and relying on our fact, we have that $\left(\bar{R}^{\prime} \theta\right) \phi \downarrow$ is a $\Sigma_{0}^{-}$-message. Therefore, relying on Lemma A.3, and since $\mu_{\phi_{S}}^{1}\left(R_{i}^{\prime}\right)<\mu_{\phi_{S}}^{1}\left(R_{i}\right)$ for $i \in I_{1} \neq \emptyset$, and $\mu_{\phi_{S}}^{1}\left(R_{i}^{\prime}\right)=\mu_{\phi_{S}}^{1}\left(R_{i}\right)$ otherwise, we deduce that:

$$
\begin{aligned}
\mu_{\phi_{S}^{-}}^{1}\left(\bar{R}^{\prime}\right) & =\operatorname{Multi}\left(\overline{R^{\prime}} \phi_{S}^{-} \downarrow\right) \\
& \leq \operatorname{Multi}\left(\operatorname{des}\left(R_{1}^{\prime} \phi_{S} \downarrow, \ldots, R_{k}^{\prime} \phi_{S} \downarrow\right)\right) \\
& =\{\text { des }\} \uplus \mu_{\phi_{S}}^{1}\left(R_{1}^{\prime}\right) \uplus \cdots \uplus \mu_{\phi_{S}}^{1}\left(R_{k}^{\prime}\right) \\
& <\{\text { des }\} \uplus \mu_{\phi_{S}}^{1}\left(R_{1}\right) \uplus \cdots \uplus \mu_{\phi_{S}}^{1}\left(R_{k}\right) \\
& =\mu_{\phi_{S}}^{1}(R) .
\end{aligned}
$$

As $\left(\bar{R}^{\prime} \theta\right) \phi \downarrow$ is a $\Sigma_{0}^{-}$-message, we deduce that $\left(\bar{R}^{\prime} \theta\right) \psi \downarrow$ is a $\Sigma_{0}^{-}$-message. Since $R^{\prime} \phi_{S} \downarrow$ is not a $\Sigma_{0}^{+}$message, Lemma 4.6 applies, and we obtain $\mu_{\phi_{S}}^{1}\left(R\left[\bar{R}^{\prime}\right]_{p}\right)<\mu_{\phi_{S}}^{1}\left(R\left[R^{\prime}\right]_{p}\right)=\mu_{\phi_{S}}^{1}(R)$.

Construction of $\overline{R^{\prime \prime}}$. Let $v_{\text {des }}^{\prime \prime}$ be the substitution such that $x v_{\text {des }}^{\prime \prime}=c$, and $y v_{\text {des }}^{\prime \prime}=c_{\text {min }}$ for any other variable $y \in \operatorname{vars}\left(\ell_{\mathrm{des}}\right)$. For $i \in\{1, \ldots, k\}$, let $R_{i}^{\prime \prime}=R_{i}$ in case $i \in I_{1}$, and $R_{i}^{\prime \prime}=t_{i} v_{\text {des }}^{\prime \prime}$ otherwise. Let $\bar{R}^{\prime \prime}=\operatorname{des}\left(R_{1}^{\prime \prime}, \ldots, R_{k}^{\prime \prime}\right)$. By construction of $\bar{R}^{\prime \prime}$, we have that $\bar{R}^{\prime \prime} \phi_{S} \downarrow$ is a $\Sigma_{0}^{+}$-message. By hypothesis, we have that $\phi_{S} \sqsubseteq_{s} \psi_{S}$, and thus we deduce that $\bar{R}^{\prime \prime} \psi_{S} \downarrow$ is a $\Sigma_{0}^{+}$-message. Then, thanks to Lemma 4.9, we deduce that $\left(\bar{R}^{\prime \prime} \theta\right) \psi \downarrow=\bar{R}^{\prime \prime} \psi_{S} \downarrow \lambda_{Q}$. Regarding the measure, we have that $\{$ des $\}<\mu_{\phi_{S}}^{1}\left(R^{\prime}\right)$, since des occurs in $R^{\prime} \phi_{S} \downarrow$. We have that $\mu_{\phi_{S}}^{1}\left(\bar{R}^{\prime \prime}\right)<\{$ des $\}$, since $\bar{R}^{\prime \prime} \phi_{S} \downarrow$ is a $\Sigma_{0}^{+}$-message. Therefore, we have that $\mu_{\phi_{S}}^{1}\left(\bar{R}^{\prime \prime}\right)<\mu_{\phi_{S}}^{1}\left(R^{\prime}\right)$. Since $R^{\prime} \phi_{S} \downarrow$ is not a $\Sigma_{0}^{+}$-message, Lemma 4.6 applies, and we obtain $\mu_{\phi_{S}}^{1}\left(R\left[\bar{R}^{\prime \prime}\right]_{p}\right)<\mu_{\phi_{S}}^{1}\left(R\left[R^{\prime}\right]_{p}\right)=\mu_{\phi_{S}}^{1}(R)$.

At this point, we have that $\bar{R}^{\prime}=\operatorname{des}\left(R_{1}^{\prime}, \ldots, R_{k}^{\prime}\right), \bar{R}^{\prime \prime}=\operatorname{des}\left(R_{1}^{\prime \prime}, \ldots, R_{k}^{\prime \prime}\right)$, and both $\left(\bar{R}^{\prime} \theta\right) \psi \downarrow$ and $\left(\bar{R}^{\prime \prime} \theta\right) \psi \downarrow$ are $\Sigma_{0}^{-}$-messages. By construction, for each $1 \leq i \leq k$, we have that either $R_{i}=R_{i}^{\prime}$ or $R_{i}=R_{i}^{\prime \prime}$. Therefore, for each $1 \leq i \leq k$, we have that $\left(R_{i} \theta\right) \psi \downarrow$ is a $\Sigma_{0}^{-}$-message and unifies with $t_{i}$. We put a $c \theta$ in atomic positions of $\ell_{\text {des }}$ in some $R_{i}^{\prime}$. As $\left(\bar{R}^{\prime} \theta\right) \psi \downarrow$ is a $\Sigma_{0}^{-}$message, it means that each of the $\left(R_{i}^{\prime} \theta\right) \psi \downarrow$ has $(c \theta) \psi \downarrow$ in atomic position of $\ell_{\text {des }}$. Similarly, we get that each of the $\left(R_{i}^{\prime \prime} \theta\right) \psi \downarrow$ has $(c \theta) \psi \downarrow$ in atomic position of $\ell_{\text {des }}$. So there is $(c \theta) \psi \downarrow$ in atomic position of $\ell_{\text {des }}$ for each $\left(R_{i} \theta\right) \psi \downarrow$. We deduce that $\left(R^{\prime} \theta\right) \psi \downarrow$ is a $\Sigma_{0}^{-}$-message.

Furthermore, we have $R_{1}=R_{1}^{\prime}$ or $R_{1}=R_{1}^{\prime \prime}$. Let $\bar{R}=\bar{R}^{\prime}$ in case $R_{1}=R_{1}^{\prime}$, and $\bar{R}=\bar{R}^{\prime \prime}$ in case $R_{1}=$ $R_{1}^{\prime \prime}$. We have that:

- $\mu_{\phi_{S}}^{1}\left(R[\bar{R}]_{p}\right)<\mu_{\phi_{S}}^{1}(R)$,

- $\left(R[\bar{R}]_{p} \theta\right) \phi \downarrow=\left(R\left[R^{\prime}\right]_{p} \theta\right) \phi \downarrow=(R \theta) \phi \downarrow$, and

- $\left(R[\bar{R}]_{p} \theta\right) \psi \downarrow=(R \theta) \psi \downarrow$.

As $\left(R[\bar{R}]_{p} \theta\right) \phi \downarrow$ is a $\Sigma_{0}^{-}$-message, by minimality $\left(R[\bar{R}]_{p} \theta\right) \psi \downarrow$ is a $\Sigma_{0}^{-}$-message, and so $(R \theta) \psi \downarrow$ is a $\Sigma_{0}^{-}$-message, which is the result we want to prove. 
Thus, we have that $R \phi_{S} \downarrow$ is a $\Sigma_{0}^{+}$-message. By $\phi_{S} \sqsubseteq_{s} \psi_{S}$, we know that $R \psi_{S} \downarrow$ is a $\Sigma_{0}^{+}$-message, and Lemma 4.9 allows us to conclude that $(R \theta) \psi \downarrow=R \psi_{S} \downarrow \lambda_{Q}$ is a $\Sigma_{0}^{-}$-message.

(2) $R$ is a $\Sigma_{0}^{+}$- recipe such that $(R \theta) \phi \downarrow$ is an atomic $\Sigma_{0}^{-}-$message.

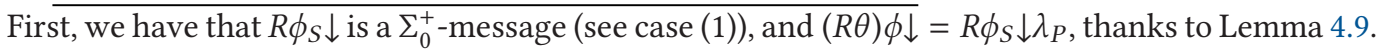
As a first step, we establish that $R \psi_{S} \downarrow$ is atomic. As $R \phi_{S} \downarrow \lambda_{P}$ is an atom, we know that $R \phi_{S} \downarrow$ is either an atom from $\Sigma_{0}^{-} \cup \mathcal{N}$, or a constant from $\Sigma_{\text {fresh. }}$.

- If $R \phi_{S} \downarrow \notin \sum_{\text {fresh }}$, then $R \phi_{S} \downarrow=R \phi_{S} \downarrow \lambda_{P}$ is atomic, and relying on our hypothesis $\phi_{S} \sqsubseteq_{s} \psi_{S}$, we deduce that $R \psi_{S} \downarrow$ is atomic.

- If $R \phi_{S} \downarrow=c \in \sum_{\text {fresh }}$, then there exists $x$ such that $x \rho=c$. Since $x \in \operatorname{dom}(\rho)$, we know that $x \notin \operatorname{dom}(m g u(\Gamma))$, and thus $x m g u(\Gamma)=x$. Therefore, we have that $x \sigma=x \sigma_{S} \lambda_{P}=$ $x(m g u(\Gamma) \rho) \lambda_{P}=(x \rho) \lambda_{P}=c \lambda_{P}=R \phi_{S} \downarrow \lambda_{P}$. As $R \phi_{S} \downarrow \lambda_{P}$ is an atom, we deduce that $x \sigma$ is atomic, and by definition of $\rho$, we have that $c=x \rho \in \sum_{\text {fresh }}^{\text {atom }}$, and thus $R \phi_{S} \downarrow$ is atomic. Relying on our hypothesis $\phi_{S} \sqsubseteq_{s} \psi_{S}$, we deduce that $R \psi_{S} \downarrow=c$ is atomic.

Since $\lambda_{Q}$ replaces atoms with atoms, we deduce in both cases that $R \psi_{S} \downarrow \lambda_{Q}$ is atomic, and thus $(R \theta) \psi \downarrow=\left(R \psi_{S} \downarrow\right) \lambda_{Q}$ (Lemma 4.9) is an atomic $\Sigma_{0}^{-}$-message.

(3) $R$ and $R^{\prime}$ are $\Sigma_{0}^{+}$-recipes, $(R \theta) \phi \downarrow,\left(R^{\prime} \theta\right) \phi \downarrow$ are $\Sigma_{0}^{-}$-messages, and $(R \theta) \phi \downarrow=\left(R^{\prime} \theta\right) \phi \downarrow$.

Step 1: We prove that $R$ and $R^{\prime}$ are simple $\Sigma_{0}^{+}$-recipes, in normal form w.r.t. $\downarrow$. Moreover, we show that $R^{\prime}$ is a subterm recipe such that $R^{\prime} \phi_{S} \downarrow$ is either an encrypted term, or a name from $\mathcal{N}$, or a constant from $\Sigma_{0}^{+}$. Regarding $R$, we show that $R$ is of the form $C\left[R_{1}, \ldots, R_{n}\right]$ where for each $i \in\{1, \ldots, n\}$, we have that $R_{i} \phi_{S} \downarrow$ is either an encrypted term, or a name from $\mathcal{N}$, or a constant from $\Sigma_{0}^{+}$.

We have that $(R \theta) \phi \downarrow$ and $\left(R^{\prime} \theta\right) \phi \downarrow$ are $\Sigma_{0}^{-}$-messages, $\mu_{\phi_{S}}(R)<\mu_{\phi_{S}}\left(R=R^{\prime}\right)$ and $\mu_{\phi_{S}}\left(R^{\prime}\right)<$ $\mu_{\phi_{S}}\left(R=R^{\prime}\right)$. Hence, we deduce that $(R \theta) \psi \downarrow$ and $\left(R^{\prime} \theta\right) \psi \downarrow$ are $\Sigma_{0}^{-}$-messages.

Assume that either $R$ or $R^{\prime}$ is not in normal form w.r.t. $\downarrow$, say $R$. Then $\mu_{\phi_{S}}^{1}(R \downarrow)<\mu_{\phi_{S}}^{1}(R)$ by Lemma 4.7. Moreover, as $R \theta \rightarrow^{*} R \Downarrow \theta$, Lemma 4.3 applies: $(R \Downarrow \theta) \phi \downarrow=(R \theta) \phi \downarrow$ and $(R \Downarrow \theta) \psi \downarrow=$ $(R \theta) \psi \downarrow$, as $(R \theta)$ gives a $\Sigma_{0}^{-}$-message in both $\phi$ and $\psi$. So $\left(R \downarrow \theta=R^{\prime} \theta\right)$ holds in $\phi$. By $\mu_{\phi_{S}}\left(R \downarrow=R^{\prime}\right)<$ $\mu_{\phi_{S}}\left(R=R^{\prime}\right)$, it transfers to $\psi$ and we deduce $(R \theta) \psi \downarrow=(R \downarrow \theta) \psi \downarrow=\left(R^{\prime} \theta\right) \psi \downarrow$, which is the result we want to establish.

Now, we assume that both $R$ and $R^{\prime}$ are in normal form w.r.t. $\downarrow$. Thus, both are simple by Lemma 4.5. In case both $R$ and $R^{\prime}$ have a constructor as root symbol, then this is necessarily the same. Therefore, we have that $R=\mathrm{f}\left(R_{1}, \ldots, R_{k}\right)$ and $R^{\prime}=\mathrm{f}\left(R_{1}^{\prime}, \ldots, R_{k}^{\prime}\right)$. We have that $\left(R_{i} \theta\right) \phi \downarrow$ and $\left(R_{i}^{\prime} \theta\right) \phi \downarrow$ are $\Sigma_{0}^{+}$-messages $(1 \leq i \leq k)$, and $\left(R_{i} \theta\right) \phi \downarrow=\left(R_{i}^{\prime} \theta\right) \phi \downarrow(1 \leq i \leq k)$. Since $\mu_{\phi_{S}}\left(R_{i}=R_{i}^{\prime}\right)<$ $\mu_{\phi_{S}}\left(R=R^{\prime}\right)$, we deduce that $\left(R_{i} \theta\right) \psi \downarrow=\left(R_{i}^{\prime} \theta\right) \psi \downarrow$, and thus $(R \theta) \psi \downarrow=\left(R^{\prime} \theta\right) \psi \downarrow$, which is the result we want to prove.

Therefore, we can assume that say $R^{\prime}$ is a subterm-recipe, and $R$ is simple, so $R=C\left[R_{1}, \ldots, R_{n}\right]$ where for each $i, R_{i}$ is a subterm-recipe. Now, assume that there is $i_{0} \operatorname{such}$ that $\operatorname{root}\left(R_{i_{0}} \phi_{S} \downarrow\right)=\mathrm{f} \in$ $\sum_{\mathrm{c}}$ and $\mathrm{f}$ is transparent, then $R_{i_{0}} \phi_{S} \downarrow=\mathrm{f}\left(C_{1}^{\mathrm{f}}\left[R_{i_{0}}\right], \ldots, C_{k}^{\mathrm{f}}\left[R_{i_{0}}\right]\right) \phi_{S} \downarrow$. We consider the context $\bar{C}$ such that $\bar{C}\left[R_{1}, \ldots, R_{n}\right]=C\left[R_{1}, \ldots, \mathrm{f}\left(C_{1}^{\mathrm{f}}\left[R_{i_{0}}\right], \ldots, C_{k}^{\mathrm{f}}\left[R_{i_{0}}\right]\right), \ldots, R_{n}\right]$. We have that $\bar{R}=\bar{C}\left[R_{1}, \ldots, R_{n}\right]$ is a $\Sigma_{0}^{+}$-recipe such that $\bar{R} \phi_{S} \downarrow=R \phi_{S} \downarrow$, and thus $\mu_{\phi_{S}}^{1}(R)=\mu_{\phi_{S}}^{1}(\bar{R})$, and $(\bar{R} \theta) \phi \downarrow=\bar{R} \phi_{S} \downarrow \lambda_{P}$ by Lemma 4.9, which gives $(\bar{R} \theta) \phi \downarrow=\bar{R} \phi_{S} \downarrow \lambda_{P}=R \phi_{S} \downarrow \lambda_{P}=(R \theta) \phi \downarrow$. We have that $\mu_{\phi_{S}}^{2}(\bar{R})<\mu_{\phi_{S}}^{2}(R)$. The equality $\bar{R} \phi_{S} \downarrow=R \phi_{S} \downarrow$ transfers to $\bar{R} \psi_{S} \downarrow=R \psi_{S} \downarrow$ by $\phi_{S} \sqsubseteq_{s} \psi_{S}$ and we deduce $(\bar{R} \theta) \psi \downarrow=(R \theta) \psi \downarrow$ by Lemma 4.9. As $\mu_{\phi_{S}}\left(\bar{R}=R^{\prime}\right)<\mu_{\phi_{S}}\left(R=R^{\prime}\right)$, the equality $(\bar{R} \theta) \phi \downarrow=\left(R^{\prime} \theta\right) \phi \downarrow$ transfers to $(\bar{R} \theta) \psi \downarrow=$ $\left(R^{\prime} \theta\right) \psi \downarrow$ and we deduce that $(R \theta) \psi \downarrow=\left(R^{\prime} \theta\right) \psi \downarrow$, which is the result we want to prove. 
Therefore, we deduce that each $R_{i} \phi_{S} \downarrow$ (with $1 \leq i \leq n$ ) is either an encrypted term, a constant from $\Sigma_{0}^{+}$, or a name from $\mathcal{N}$. A similar reasoning allows us to establish that $R^{\prime} \phi_{S} \downarrow$ is either an encrypted term, a constant from $\Sigma_{0}^{+}$, or a name from $\mathcal{N}$.

Step 2: We now establish that $(R \theta) \psi \downarrow=\left(R^{\prime} \theta\right) \psi \downarrow$.

Let $t=R \phi_{S} \downarrow$ and $v=R^{\prime} \phi_{S} \downarrow$. By Lemma 4.9 and $(R \theta) \phi \downarrow=\left(R^{\prime} \theta\right) \phi \downarrow$, we know that $t \lambda_{P}=v \lambda_{P}$. If $t=v$, then we have that $R \phi_{S} \downarrow=R^{\prime} \phi_{S} \downarrow$, since $\phi_{S} \sqsubseteq_{s} \psi_{S}$. Then by Lemma 4.9, we deduce that $(R \theta) \psi \downarrow=\left(R^{\prime} \theta\right) \psi \downarrow$.

From now on, we assume that $t \neq v$. Since $t \neq v$, we know that there exists a position $p$ defined in $t$ and $v$ such that $\operatorname{root}\left(\left.t\right|_{p}\right) \neq \operatorname{root}\left(\left.v\right|_{p}\right)$. Let $p$ be any position defined in $t$ and $v$ such that $\operatorname{root}\left(\left.t\right|_{p}\right) \neq$ $\operatorname{root}\left(\left.v\right|_{p}\right)$. Since $t \lambda_{P}=v \lambda_{P}$, and $\operatorname{dom}\left(\lambda_{P}\right) \subseteq \Sigma_{\text {fresh }}$, we have that $\left.t\right|_{p} \in \Sigma_{\text {fresh }}$ or $\left.v\right|_{p} \in \Sigma_{\text {fresh }}$.

We first assume that there exists such a position $p$ that falls outside the context $C$. More precisely, we have that $p=p^{\prime} \cdot p^{\prime \prime}$ (with $p^{\prime}$ a strict prefix of $p$ ) and $\left.C\left[R_{1}, \ldots, R_{n}\right]\right|_{p^{\prime}}=R_{i_{0}}$ for some $i_{0} \in\{1, \ldots, n\}$. Therefore, since $R_{i_{0}} \phi_{S} \downarrow$ is not a leaf $\left(p^{\prime \prime} \neq \epsilon\right)$, we know that $\left.t\right|_{p^{\prime}}=R_{i_{0}} \phi_{S} \downarrow$ is an encrypted subterm of $\phi_{S}$. Relying on Lemma 4.10, we have that:

- $\left.t\right|_{p^{\prime}} \in \operatorname{ESt}\left(\phi_{S}\right) \subseteq E S t\left(\mathcal{K}_{S} \sigma_{S}\right) \subseteq E S t\left(\mathcal{K}_{P}(m g u(\Gamma) \rho)\right) \subseteq E S t\left(\mathcal{K}_{P}\right) \sigma_{S}$.

- $\left.v\right|_{p^{\prime}} \in \operatorname{ESt}\left(\phi_{S}\right) \subseteq E S t\left(\mathcal{K}_{S} \sigma_{S}\right) \subseteq E S t\left(\mathcal{K}_{P}(m g u(\Gamma) \rho)\right) \subseteq E S t\left(\mathcal{K}_{P}\right) \sigma_{S}$.

This allows us to conclude that there exist $t^{\prime}, v^{\prime} \in \operatorname{ESt}\left(\mathcal{K}_{P}\right)$ such that $t^{\prime} \sigma_{S}=\left.t\right|_{p^{\prime}}$, and $v^{\prime} \sigma_{S}=\left.v\right|_{p^{\prime}}$. Since $t \lambda_{P}=v \lambda_{P}$, we know that $\left.\left(t \lambda_{P}\right)\right|_{p^{\prime}}=\left.\left(v \lambda_{P}\right)\right|_{p^{\prime}}$, thus $\left.t\right|_{p^{\prime}} \lambda_{P}=\left.v\right|_{p^{\prime}} \lambda_{P}$, and $\left(t^{\prime} \sigma_{S}\right) \lambda_{P}=\left(v^{\prime} \sigma_{S}\right) \lambda_{P}$. We have that $\left(t^{\prime} \sigma_{S}\right) \lambda_{P}=t^{\prime}\left(\sigma_{S} \lambda_{P}\right)$ and also that $\left(v^{\prime} \sigma_{S}\right) \lambda_{P}=v^{\prime}\left(\sigma_{S} \lambda_{P}\right)$. Since we have that $\sigma=\sigma_{S} \lambda_{P}$, we deduce that $t^{\prime} \sigma=v^{\prime} \sigma$. By definition of $\sigma_{S}$, we have that $t^{\prime} \sigma_{S}=v^{\prime} \sigma_{S}$. Thus, we have that $\left.t\right|_{p^{\prime}}=$ $\left.v\right|_{p^{\prime}}$ leading to a contradiction, since we have assumed that $t$ and $v$ differ below the position $p^{\prime}$.

Thus, we know that for any position $p$ defined in $t$ and $v$ such that $\operatorname{root}\left(\left.t\right|_{p}\right) \neq \operatorname{root}\left(\left.v\right|_{p}\right)$, we have that $\left.t\right|_{p}$ or $\left.v\right|_{p}$ is in $\Sigma_{\text {fresh }}$, and $p$ is a position of $C$.

If $\left.t\right|_{p}=c \in \Sigma_{\text {fresh }}$, let $\bar{R}=R[c \theta]_{p}$. We have that $\mu_{\phi_{S}}^{1}(\bar{R})<\mu_{\phi_{S}}^{1}(R)$, since $\mu_{\phi_{S}}^{1}(c \theta)<\mu_{\phi_{S}}^{1}\left(\left.R\right|_{p}\right)$ (note that $\left.R\right|_{p} \phi_{S} \downarrow=c$, whereas $(c \theta) \phi_{S} \downarrow$ is a $\Sigma_{0}^{+}$-message, and it is an atom when $\left.c \in \sum_{\text {fresh }}^{\text {atom }}\right)$. Moreover, we have that:

- $(\bar{R} \theta) \phi \downarrow=(R \theta) \phi \downarrow=\left(R^{\prime} \theta\right) \phi \downarrow$, and

- $\mu_{\phi_{S}}\left(\bar{R}=R^{\prime}\right)<\mu_{\phi_{S}}\left(R=R^{\prime}\right)$.

Thus, by induction hypothesis, we have that $(\bar{R} \theta) \psi \downarrow=\left(R^{\prime} \theta\right) \psi \downarrow$. We also have $\left.R\right|_{p} \phi_{S} \downarrow=c$ so by $\phi_{S} \sqsubseteq_{s} \psi_{S}$, we know that $\left.R\right|_{p} \psi_{S} \downarrow=c$. By Lemma 4.9, $\left(\left.R\right|_{p} \theta\right) \psi \downarrow=(c \theta) \psi \downarrow$. So $(\bar{R} \theta) \psi \downarrow=(R \theta) \psi \downarrow$. We deduce $(R \theta) \psi \downarrow=\left(R^{\prime} \theta\right) \psi \downarrow$, which is the result we want to prove.

Hence, from now on, we assume that $\left.t\right|_{p} \notin \sum_{\text {fresh }}$, and thus $\left.v\right|_{p}=c$ for some $c \in \sum_{\text {fresh. }}$. Let $p_{1}, \ldots, p_{m}$ be the positions such that for each $i \in\{1, \ldots, m\}$, we have that $p_{i}$ is defined in both $t$ and $v$, and $\operatorname{root}\left(\left.t\right|_{p_{i}}\right) \neq \operatorname{root}\left(\left.v\right|_{p_{i}}\right)$. For each $i \in\{1, \ldots, m\}$, we know that $p_{i}$ is a position of $C$ such that $\left.t\right|_{p_{i}} \notin \sum_{\text {fresh }}$, and $\left.v\right|_{p_{i}} \in \sum_{\text {fresh }}$. We denote $c_{\text {fresh }}^{i}$ the constant from $\sum_{\text {fresh }}$ such that $\left.v\right|_{p_{i}}=c_{\text {fresh }}^{i}$. We have $\left(c_{\text {fresh }}^{i} \theta\right) \phi \downarrow=\left(\left.R\right|_{p_{i}} \theta\right) \phi \downarrow$. $\mu_{\phi_{S}}^{1}\left(c_{\text {fresh }}^{i}\right)<\mu_{\phi_{S}}^{1}\left(R^{\prime}\right)$ and $\mu_{\phi_{S}}^{1}\left(\left.R\right|_{p_{i}}\right) \leq \mu_{\phi_{S}}^{1}(R)$ so $\mu_{\phi_{S}}\left(c_{\text {fresh }}^{i}=\left.R\right|_{p_{i}}\right)<$ $\mu_{\phi_{S}}\left(R=R^{\prime}\right)$. We deduce $\left(c_{\text {fresh }}^{i} \theta\right) \psi \downarrow=\left(\left.R\right|_{p_{i}} \theta\right) \psi \downarrow$.

Now, let $\bar{C}$ be the context obtained from $C$ by putting $c_{\text {fresh }}^{i}$ at position $p_{i}$ for each $i$. Let $\bar{R}=$ $\bar{C}\left[R_{1}, \ldots, R_{n}\right] .(\bar{R} \theta) \psi \downarrow=(R \theta) \psi \downarrow$ by $\left(c_{\text {fresh }}^{i} \theta\right) \psi \downarrow=\left(\left.R\right|_{p_{i}} \theta\right) \psi \downarrow$. Furthermore, we have that $\bar{R} \phi_{S} \downarrow=$ $R^{\prime} \phi_{S} \downarrow$ by construction. By $\phi_{S} \sqsubseteq_{s} \psi_{S}$, we get $\bar{R} \psi_{S} \downarrow=R^{\prime} \psi_{S} \downarrow$. By Lemma 4.9, we get $(\bar{R} \theta) \psi \downarrow=$ $\left(R^{\prime} \theta\right) \psi \downarrow$, and thus $(R \theta) \psi \downarrow=\left(R^{\prime} \theta\right) \psi \downarrow$, which is the result we want to prove.

We can conclude with the proof of our main theorem on equivalence. 
TheOREM 3.9. Let $\mathcal{K}_{P}$ be a $\Sigma_{0}^{-}$-configuration type-compliant w.r.t. $\left(\mathcal{T}_{0}, \delta_{0}\right)$ and $\mathcal{K}_{Q}$ be another $\Sigma_{0}^{-}$-configuration. We have that $\mathcal{K}_{P} \nsubseteq_{t} \mathcal{K}_{Q}$ w.r.t. $\Sigma_{0}^{-}$with witness $(\mathrm{tr}, \phi)$ if, and only if, there exists a witness $\left(\operatorname{tr}^{\prime}, \phi^{\prime}\right) \in$ trace $_{\Sigma_{0}^{+}}\left(\mathcal{K}_{P}\right)$ of this non-inclusion such that its underlying execution $\mathcal{K}_{P} \stackrel{\operatorname{tr}^{\prime}}{\Longrightarrow}$ $\left(\mathcal{P} ; \phi^{\prime} ; \sigma ; i\right)$ w.r.t. $\Sigma_{0}^{+}$is well-typed and $\overline{\mathrm{tr}}=\overline{\mathrm{tr}^{\prime}}$.

Proof. The converse part has already been discussed in Section 3.3.2. Thus, we now prove the direct part. Let $\mathcal{K}_{P}$ be a $\Sigma_{0}^{-}$-configuration type-compliant w.r.t. $\left(\mathcal{T}_{0}, \delta_{0}\right)$ and $\mathcal{K}_{Q}$ be a $\Sigma_{0}^{-}$configuration. Assume $\mathcal{K}_{P} \nsubseteq_{t} \mathcal{K}_{Q}$ w.r.t. $\Sigma_{0}^{-}$. Let $(\operatorname{tr}, \phi) \in \operatorname{trace}_{\Sigma_{0}^{-}}\left(\mathcal{K}_{P}\right)$ with underlying substitution $\sigma$ be a witness of non-inclusion. Thanks to Proposition 5.4, we know that there exists $\left(\operatorname{tr}_{S}, \phi_{S}\right) \in \operatorname{trace}_{\Sigma_{0}^{+}}\left(\mathcal{K}_{P}\right)$ a witness of this non-inclusion with underlying substitution $\sigma_{S}$ such that $\sigma_{S}=m g u(\Gamma) \rho$, as well as two substitutions $\lambda_{P}$ and $\theta$ such that:

- $\Gamma=\left\{(u, v) \mid u, v \in E S t\left(\mathcal{K}_{P}\right)\right.$ such that $\left.u \sigma=v \sigma\right\}$.

- $\rho$ is a bijective renaming from variables in $\operatorname{dom}(\sigma) \backslash \operatorname{dom}(m g u(\Gamma))$ to constants in $\sum_{\text {fresh }}$ such that $x \rho \in \Sigma_{\text {fresh }}^{\text {atom }}$ if, and only if, $x \sigma$ is an atomic $\Sigma_{0}^{-}$-message.

- $\operatorname{dom}(\theta) \subseteq \sum_{\text {fresh }}$, for any $c \in \sum_{\text {fresh }}$ occurring in $\operatorname{tr}_{S}$, we have that $c \in \operatorname{dom}(\theta), c \theta \in$ $\mathcal{T}\left(\Sigma, \Sigma_{0}^{-} \uplus \operatorname{dom}\left(\phi_{S}\right)\right)$, and $\mathrm{w}^{\prime} \prec c$ for any $\mathrm{w}^{\prime} \in \operatorname{vars}(c \theta)$ (where $\prec$ is the ordering induced by $\left.\operatorname{tr}_{S}\right)$.

- for any $c \in \operatorname{dom}(\theta),(c \theta) \phi_{S} \downarrow$ is a $\Sigma_{0}^{+}$-message, and it is an atom when $c \in \Sigma_{\text {fresh }}^{\text {atom }}$.

- $\lambda_{P}$ is the first-order substitution associated to $\theta$ through $\phi_{S}$.

- $\phi=\phi_{S} \lambda_{P}, \sigma=\sigma_{S} \lambda_{P}$, and $\left(\operatorname{tr}_{S} \theta\right) \phi \downarrow=\operatorname{tr} \phi \downarrow$.

First, since $\mathcal{K}_{P}$ is type-compliant, we know that encrypted subterms in $\operatorname{ESt}\left(\mathcal{K}_{P}\right)$ that are unifiable have the same type. Then, by definition of a typing system, this allows us to deduce that $m g u(\Gamma)$ is well-typed. Since we have enough constants of each type, we may assume w.l.o.g. that $\rho$ is also well-typed. Hence, we have that $\sigma_{S}=m g u(\Gamma) \rho$ is well-typed, which means that $\left(\operatorname{tr}_{S}, \phi_{S}\right)$ is a welltyped witness of the non-inclusion $\mathcal{K}_{P} \nsubseteq_{t} \mathcal{K}_{Q}$ w.r.t. $\Sigma_{0}^{+}$. As $\left(\operatorname{tr}_{S} \theta\right) \phi \downarrow=\operatorname{tr} \phi \downarrow$, we have $\overline{\operatorname{tr}_{S}}=\overline{\operatorname{tr}}$. This concludes the proof.

\section{REFERENCES}

[1] M. Abadi and C. Fournet. 2001. Mobile values, new names, and secure communication. In Proceedings of the 28th Symposium on Principles of Programming Languages (POPL'01). ACM Press.

[2] M. Abadi and R. M. Needham. 1996. Prudent engineering practice for cryptographic protocols. IEEE Trans. Softw. Eng. 22, 1 (1996), 6-15.

[3] Ben Adida. 2008. Helios: Web-based open-audit voting. In Proceedings of the 17th USENIX Security Symposium. USENIX Association, 335-348.

[4] O. Almousa, S. Mödersheim, P. Modesti, and L. Viganò. 2015. Typing and compositionality for security protocols: A generalization to the geometric fragment. In Proceedings of the 20th European Symposium on Research in Computer Security (ESORICS'15).

[5] M. Arapinis, V. Cheval, and S. Delaune. 2015. Composing security protocols: From confidentiality to privacy. In Proceedings of the 4th International Conference on Principles of Security and Trust (POST'15) (Lecture Notes in Computer Science), Vol. 9036. Springer, London, UK, 324-343.

[6] M. Arapinis, T. Chothia, E. Ritter, and M. Ryan. 2010. Analysing unlinkability and anonymity using the applied pi calculus. In Proceedings of the 23rd Computer Security Foundations Symposium (CSF'10). IEEE Computer Society Press, 107-121.

[7] M. Arapinis and M. Duflot. 2007. Bounding messages for free in security protocols. In Proceedings of the 27th Conference on Foundations of Software Technology and Theoretical Computer Science (FSTTCS'07).

[8] A. Armando, D. Basin, Y. Boichut, Y. Chevalier, L. Compagna, J. Cuellar, P. Hankes Drielsma, P.-C. Héam, O. Kouchnarenko, J. Mantovani, S. Mödersheim, D. von Oheimb, M. Rusinowitch, J. Santiago, M. Turuani, L. Viganò, and L. Vigneron. 2005. The AVISPA tool for the automated validation of Internet security protocols and applications. In Proceedings of the 17th International Conference on Computer Aided Verification (CAV'2005) (LNCS), Vol. 3576. 281-285. 
[9] M. Backes, C. Hritcu, and M. Maffei. 2008. Automated verification of remote electronic voting protocols in the applied pi-calculus. In Proceedings of the 21st IEEE Computer Security Foundations Symposium (CSF'08). IEEE Computer Society.

[10] David Basin, Jannik Dreier, Lucca Hirschi, Saša Radomirovic, Ralf Sasse, and Vincent Stettler. 2018. A formal analysis of $5 \mathrm{G}$ authentication. In Proceedings of the 25th ACM Conference on Computer and Communications Security (CCS'18), Vol. 14. ACM Press.

[11] M. Baudet. 2005. Deciding security of protocols against off-line guessing attacks. In Proceedings of the12th ACM Conference on Computer and Communications Security (CCS'05). ACM Press.

[12] B. Blanchet. 2001. An efficient cryptographic protocol verifier based on prolog rules. In Proceedings of the 14th Computer Security Foundations Workshop (CSFW'01). IEEE Computer Society Press.

[13] B. Blanchet. 2008. Vérification automatique de protocoles cryptographiques: Modèle formel et modèle calculatoire. (Automatic verification of security protocols: Formal model and computational model.) Mémoire d'habilitation à diriger des recherches. Université Paris-Dauphine.

[14] B. Blanchet, M. Abadi, and C. Fournet. 2008. Automated verification of selected equivalences for security protocols. 7. Logic. Alg. Prog. 75, 1 (2008), 3-51.

[15] B. Blanchet and A. Podelski. 2003. Verification of cryptographic protocols: Tagging enforces termination. In Foundations of Software Science and Computation Structures (FoSSaCS'03).

[16] Bruno Blanchet and Ben Smyth. 2018. Automated reasoning for equivalences in the applied pi calculus with barriers. f. Comput. Sec. 26, 3 (2018), 367-422.

[17] M. Bruso, K. Chatzikokolakis, and J. den Hartog. 2010. Formal verification of privacy for RFID systems. In Proceedings of the 23rd Computer Security Foundations Symposium (CSF'10).

[18] Mayla Brusó, Konstantinos Chatzikokolakis, Sandro Etalle, and Jerry Den Hartog. 2012. Linking unlinkability. In Proceedings of the 7th International Symposium on Trustworthy Global Computing (TGC'12), Vol. 8191. Springer, 129144. DOI : https://doi.org/10.1007/978-3-642-41157-1_9

[19] R. Chadha, Ş. Ciobâcă, and S. Kremer. 2012. Automated verification of equivalence properties of cryptographic protocols. In Proceedings of the 21st European Symposium on Programming (ESOP'12) (LNCS).

[20] V. Cheval, H. Comon-Lundh, and S. Delaune. 2011. Trace equivalence decision: Negative tests and non-determinism. In Proceedings of the 18th ACM Conference on Computer and Communications Security (CCS'11). ACM.

[21] V. Cheval, V. Cortier, and S. Delaune. 2013. Deciding equivalence-based properties using constraint solving. Theoret. Comput. Sci. 492 (June 2013), 1-39.

[22] R. Chrétien, V. Cortier, and S. Delaune. 2013. From security protocols to pushdown automata. In Proceedings of the 40th International Colloquium on Automata, Languages and Programming (ICALP'13).

[23] R. Chrétien, V. Cortier, and S. Delaune. 2014. Typing messages for free in security protocols: The case of equivalence properties. In Proceedings of the 25th International Conference on Concurrency Theory (CONCUR'14) (Lecture Notes in Computer Science). Springer.

[24] R. Chrétien, V. Cortier, and S. Delaune. 2015. Decidability of trace equivalence for protocols with nonces. In Proceedings of the 28th IEEE Computer Security Foundations Symposium (CSF'15). IEEE Computer Society Press.

[25] Ş. Ciobâcă and V. Cortier. 2010. Protocol composition for arbitrary primitives. In Proceedings of the 23rd IEEE Computer Security Foundations Symposium (CSF'10). IEEE Computer Society Press, 322-336.

[26] H. Comon-Lundh and V. Cortier. 2003. New decidability results for fragments of first-order logic and application to cryptographic protocols. In Proceedings of the 14th International Conference on Rewriting Techniques and Applications (RTA'2003) (LNCS), Vol. 2706. Springer.

[27] H. Comon-Lundh, V. Cortier, and E. Zalinescu. 2010. Deciding security properties for cryptographic protocols. Application to key cycles. ACM Trans. Comput. Logic 11, 4 (2010)

[28] V. Cortier, A. Dallon, and S. Delaune. 2017. SAT-Equiv: An efficient tool for equivalence properties. In Proceedings of the 30th IEEE Computer Security Foundations Symposium (CSF'17). IEEE Computer Society Press.

[29] V. Cortier and S. Delaune. 2009. Safely composing security protocols. Form. Meth. Syst. Des. 34, 1 (Feb. 2009), 1-36.

[30] Véronique Cortier and Ben Smyth. 2013. Attacking and fixing Helios: An analysis of ballot secrecy. F. Comput. Sec. 21, 1 (2013), 89-148.

[31] C. Cremers. 2008. The Scyther tool: Verification, falsification, and analysis of security protocols. In Proceedings of the Conference on Computer Aided Verification (CAV'08) (LNCS), Vol. 5123/2008. Springer, 414-418.

[32] S. Delaune, S. Kremer, and M. D. Ryan. 2008. Verifying privacy-type properties of electronic voting protocols. 7 . Comput. Sec. 4 (July 2008), 435-487.

[33] N. Durgin, P. Lincoln, J. Mitchell, and A. Scedrov. 1999. Undecidability of bounded security protocols. In Proceedings of the Workshop on Formal Methods and Security Protocols.

[34] J. D. Guttman and F. Javier Thayer. 2000. Protocol independence through disjoint encryption. In Proceedings of the 13th Computer Security Foundations Workshop (CSFW'00). IEEE Comp. Soc. Press. 
[35] J. Heather, G. Lowe, and S. Schneider. 2003. How to prevent type flaw attacks on security protocols. F. Comput. Secur. 11,2 (2003), 217-244.

[36] A. V. Hess and S. Mödersheim. 2017. Formalizing and proving a typing result for security protocols in Isabelle/HOL. In Proceedings of the 30th IEEE Computer Security Foundations Symposium (CSF'17).

[37] G. Lowe. 1996. Breaking and fixing the Needham-Schroeder public-key protocol using FDR. In Proceedings of the Conference on Tools and Algorithms for the Construction and Analysis of Systems (TACAS'96) (LNCS), Vol. 1055. SpringerVerlag, 147-166.

[38] G. Lowe. 1998. Towards a completeness result for model checking of security protocols. In Proceedings of the 11th Computer Security Foundations Workshop (CSFW'98). IEEE Computer Society Press.

[39] J. Millen and V. Shmatikov. 2001. Constraint solving for bounded-process cryptographic protocol analysis. In Proceedings of the 8th ACM Conference on Computer and Communications Security (CCS'01). ACM Press.

[40] R. Ramanujam and S. P. Suresh. 2003. Tagging makes secrecy decidable with unbounded nonces as well. In Proceedings of the 3rd Conference on Foundations of Software Technology and Theoretical Computer Science (FSTTCS'03) (LNCS). Springer, 363-374.

[41] M. Rusinowitch and M. Turuani. 2003. Protocol insecurity with finite number of sessions and composed keys is NPcomplete. Theor. Comput. Sci. 299 (Apr. 2003), 451-475.

[42] B. Schmidt, S. Meier, C. Cremers, and D. Basin. 2012. Automated analysis of Diffie-Hellman protocols and advanced security properties. In Proceedings of the 25th IEEE Computer Security Foundations Symposium (CSF'12). 78-94.

[43] A. Tiu and J. E. Dawson. 2010. Automating open bisimulation checking for the Spi calculus. In Proceedings of the 23rd IEEE Computer Security Foundations Symposium (CSF'10). 307-321.

Received March 2018; revised April 2019; accepted July 2019 